\title{
Bifurcação de Poincaré-Andronov-Hopf para difeomorfismos do plano.
}

\author{
Pricila da Silva Barbosa
}

\author{
DisSERTAÇÃO APRESENTADA \\ $\mathrm{AO}$ \\ Instituto de Matemática e Estatística \\ Universidade de SÃo Paulo \\ PARA \\ OBTENÇÃO DO TÍTULO \\ Mestre EM CiênCIAS
}

Programa: Matemática Aplicada

Orientador: Prof. Dr. Antônio Luiz Pereira

Durante o desenvolvimento deste trabalho o autor recebeu auxílio financeiro do CNPq.

São Paulo, de 2010. 


\title{
Bifurcação de Poincaré-Andronov-Hopf para difeomorfismos do plano.
}

\author{
Esta versão definitiva da dissertação \\ contém as correções e alterações sugeridas pela \\ Comissão Julgadora durante a defesa realizada \\ por Pricila da Silva Barbosa em /05/2010.
}

Comissão Julgadora:

- Prof. Dr. Antônio Luiz Pereira (orientador) - IME-USP

- Prof. Dr. Marcone Corrêa Pereira - IME-USP

- Prof. Dr. Luiz Augusto da Costa Ladeira - ICMC-USP 


\section{Agradecimentos}

Agradeço...

Primeiramente a minha família, em especial à minha mãe por me incentivar nos estudos e me apoiar nos momentos difíceis.

Ao meu orientador Prof. Dr. Antônio Luiz Pereira, por toda a paciência e dedicação ao passar horas e horas me ajudando para que tudo isso fosse possível.

A todos os meus amigos do IME, em especial à Catalina Rua e Juan Fernando Zapata, por me ajudarem durante o mestrado e de alguma forma neste trabalho. 



\section{Resumo}

O objetivo principal deste trabalho é apresentar uma exposição detalhada do Teorema de Poincaré-Andronov-Hopf para uma família de transformações no plano, baseada em um trabalho de O. Lanford [1]. Este teorema dá condições para o aparecimento de uma curva fechada invariante para o fluxo quando o parâmetro passa por um determinado valor.

Apresentamos também uma aplicação a um sistema dinâmico que modela a evolução do preço e excesso de demanda em um mercado constituído por uma única mercadoria.

Palavras-chave: bifurcação, forma normal, curva fechada invariante, estabilidade. 


\section{Abstract}

The main purpose of this work is to present a detailed exposition of the Poincaré-Andronov-Hopf Theorem for a family of transformations in the plane, based on a work of O. Lanford [1]. This theorem gives conditions for the appearance of a closed invariant curve under the flow, when the parameter crosses a certain value.

We also present an application to a dynamical system modelling the evolution of the price and the excess demand in a single asset market.

Keywords: bifurcation, normal form, closed invariant curve, stability. 



\section{SUMÁRIO}

1. Preliminares. 3

1.1. Sistemas Dinâmicos. . . . . . . . . . . . . . . . . . . . 3

1.2. Conjuntos $\alpha$-limite e $w$-limite de uma órbita. . . . . . . . . . . . . . . 5

1.3. O Teorema da Bifurcação de Hopf em $\mathbb{R}^{2}$ e $\mathbb{R}^{n}$. . . . . . . . . . . . . . . . . 5

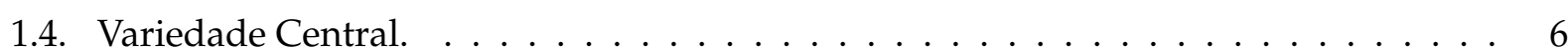

2. Bifurcação de Poincaré-Andronov-Hopf para Difeomorfismos do Plano. 9

2.1. Difeomorfismos do Plano. . . . . . . . . . . . . . . . . . . . . . . 9

2.2. Forma Canônica da Aplicação $\Phi_{\mu} . \ldots \ldots \ldots \ldots$. . . . . . . . . 10

2.3. Bifurcação de Poincaré-Andronov-Hopf. . . . . . . . . . . . . . . . . . . . 25

3. Uma Aplicação. $\quad 43$

3.1. Introdução . . . . . . . . . . . . . . . . . . . . . . . . . . . . . . 44

3.2. A Unicidade do Equilíbrio e Propriedades Básicas de Estabilidade. . . . . . . . . . . 45

3.3. Bifurcação de Hopf e Órbitas Periódicas. . . . . . . . . . . . . . . . . . . . . . 52

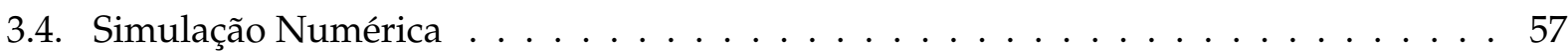





\section{Introdução}

O propósito desse trabalho é discutir o Teorema de Poincaré-Andronov-Hopf para difeomorfismos do plano, fazer uma aplicação e em seguida uma simulação numérica. Em 1942, Hopf estabeleceu condições para a ocorrência de um tipo de bifurcação num sistema n-dimensional. Entretanto, esse tipo de bifurcação já havia sido sugerido por Poincaré, em 1892, e estudado por Andronov, em 1929, para um sistema bidimensional, sendo por isto geralmente conhecida como Bifurcação de Poincaré-Andronov-Hopf. Esse teorema descreve o que acontece quando um ponto de equilíbrio de uma equação diferencial dependendo de um parâmetro passa de estável a instável, em um valor crítico do parâmetro. Um trabalho realizado por Ruelle e Takens [4] estende a teoria da bifurcação de Hopf para transformações. Eles estavam interessados em estudar o aparecimento de um toro invariante estável que, sob algumas condições, aparece nas proximidades de uma órbita fechada instável.

A prova feita por Ruelle e Takens passa por demonstrar o Teorema de Poincaré-Andronov-Hopf para a transformação de Poincaré. Dado um campo de vetores $\mathcal{X}$ e uma órbita fechada $\gamma$ do fluxo $\psi_{t}$ de $\mathcal{X}$, a idéia é considerar a transformação de Poincaré $\mathcal{P}$ associado a $\gamma$. Supondo que exista uma circunferência $\sigma$ que é invariante sob $\mathcal{P}$, então $\bigcup_{t} \psi_{t}(\sigma)$ é um toro invariante para o fluxo de $X$. Sejam $x_{\mu}$ uma família de campos de vetores e $\gamma_{\mu}$ uma família contínua de órbitas fechadas desses campos. É possível que exista um $\mu_{0}$ de tal forma que $\gamma_{\mu}$ seja estável para $\mu<\mu_{0}$ e instável para $\mu>\mu_{0}$, e um toro invariante estável se forme nesse local. Sabemos que $\gamma_{\mu}$ é estável se os autovalores da derivada da transformação de Poincaré $\mathcal{P}_{\mu}$ têm autovalores com módulo menor do que 1 (e 
instável se algum dos autovalores tem módulo maior do que 1). O teorema de bifurcação de Hopf para difeomorfismos dá condições para o aparecimento do toro, após a perda de estabilidade de $\gamma_{\mu}$.

Nossa atenção será focalizada no trabalho de Lanford [1], onde o estudo é feito para difeomorfismos do plano em geral, e não para o caso particular da transformação de Poincaré. Consideraremos neste trabalho uma família de transformações do plano $\Phi_{\mu}$, de classe $C^{4}$, a um parâmetro real, em uma vizinhança do zero no espaço de Banach $z$ em $z$, com $\Phi_{\mu}(0)=0$ para todo $\mu$. Queremos observar o que acontece quando $\mu$ varia e a origem muda de estável a um ponto fixo instável de $\Phi_{\mu}$, isto é, algum dos autovalores do espectro de $D \Phi_{\mu}(0)$ cruza a circunferência unitária. Isto pode acontecer de várias maneiras, mas consideraremos apenas o caso de um único par complexo conjugado de autovalores simples não reais; assumiremos que o restante do espectro de $D \Phi_{\mu}(0)$ se encontra estritamente dentro da circunferência unitária, e para simplificar a notação, assumiremos também que isso ocorre em $\mu=0$.

No primeiro capítulo, abordaremos definições e resultados importantes sobre sistemas dinâmicos. Também enunciaremos sem demonstração o Teorema de Variedade Central, que será muito importante para reduzirmos nosso estudo a uma variedade de dimensão 2. No segundo capítulo enunciaremos e demonstraremos o Teorema de Poincaré-Andronov-Hopf em detalhe e demonstraremos também uma proposição que dá uma condição necessária e suficiente para o surgimento da bifurcação em questão. Finalmente, no terceiro e último capítulo, trabalharemos com um difeomorfismo do plano cujas órbitas imitam a evolução do preço e do excesso de demanda de uma dada mercadoria. Para ilustrar o aparecimento de oscilações periódicas desse modelo do mercado financeiro, faremos simulações numéricas para alguns valores dos parâmetros. 


\section{CAPÍTULO 1}

\section{Preliminares.}

A teoria de Sistemas Dinâmicos estuda o comportamento a longo prazo da evolução de um sistema. A moderna teoria de sistemas dinâmicos originou-se no final do século 19, procurando responder questões fundamentais relativas a estabilidade e evolução do sistema solar, o que levou ao desenvolvimento de um campo rico e poderoso, com aplicações a física, biologia, meteorologia, astronomia, economia e outras áreas. Por analogia com a mecânica celeste, a evolução de um estado particular de um sistema dinâmico é referido como uma órbita. Um certo número de temas aparecem repetidamente no estudo de sistemas dinâmicos: existência e estabilidade de equilíbrios e órbitas periódicas, comportamento assintótico, estabilidade por pertubações, etc.

\subsection{Sistemas Dinâmicos.}

Um sistema dinâmico contínuo consiste em um espaço métrico $X$ e uma família de transformações a um parâmetro $\left\{T_{\mu}: X \rightarrow X\right\}$, com $\mu \in \mathbb{R}$ ou $\mu \in \mathbb{R}_{0}^{+}$, que forma um grupo ou semigrupo a um parâmetro, ou seja, $T_{\mu_{1}+\mu_{2}}=T_{\mu_{1}} \circ T_{\mu_{2}}$ e $T^{0}=I d$. O sistema dinâmico é chamado de fluxo se $\mu$ varia em $\mathbb{R}$ e um semi-fluxo se $\mu$ varia em $\mathbb{R}_{0}^{+}$.

Definição 1.1 Um sub-conjunto $A \in X$ é invariante para o sistema dinâmico $T_{\mu}$ se, para todo $x \in A, T_{\mu}(x)$ está definido e pertence a A para todo $\mu \in \mathbb{R}$. 
Um sistema dinâmico discreto consiste de um espaço métrico $X$ e uma função $T: X \rightarrow X$. Para $n \in \mathbb{N}$, a n-ésima iteração de $T$ é a n-ésima composição $T^{n}=T \circ \cdots \circ T$ (n vezes) e definimos $T^{0}$ como a função identidade, denotada por Id. Se $T$ é inversível, então $T^{-n}=T^{-1} \circ \cdots \circ T^{-1}$ (n vezes). Como $T^{n+m}=T^{n} \circ T^{m}$, essas iterações formam um grupo se $T$ é inversível. Embora tenhamos definido sistemas dinâmicos de maneira bem geral, $X$ tem muitas vezes uma estrutura adicional que é preservada pela função $T$. Por exemplo, $(X, T)$ pode ser um espaço de medida e uma transformação que preserva medida; um espaço topológico e uma transformação contínua; um espaço métrico e uma isometria, ou uma variedade diferenciável e uma transformação diferenciável.

Definição 1.2 Se $T$ é uma transformação em $X$, o ponto $x$ é um ponto fixo para $T$ se $T(x)=x$. O ponto $x$ é periódico de período $n$ se $T^{n}(x)=x$. O menor positivo $n$ para cada $T^{n}(x)=x$ é chamado de primeiro período de $x$. O conjunto de todas as iterações de um ponto periódico é denominado uma órbita periódica.

Exemplo 1.1 Considerando o sistema dinâmico discreto sobre a reta real $\mathbb{R}$ definido pelas iterações da aplicação $T: x \longmapsto-x$, temos que o ponto 0 é um ponto fixo.

Definição 1.3 Seja $X$ um espaço métrico e $T: X \longrightarrow X$ uma transformação. Dizemos que um ponto fixo fixo $p$ de $T$ é atrator se $T(p)=p$ e $T^{n}(x)=p$ quando $n \longrightarrow \infty$, para todo $x \in X$.

O resultado que segue é frequentemente usado para provar a existência de pontos fixos.

Teorema 1.1 Seja X um espaço métrico completo. Se $T: X \longrightarrow X$ é contínua e, para algum $m, T^{m}$ é uma contração, então existe um único ponto fixo $p$ para T. Mais ainda, $p$ é atrator.

A demonstração do Teorema 1.1 pode ser visto em [7]. O teorema seguinte é conhecido como Método Indireto de Lyapunov.

Teorema 1.2 Seja T uma transformação de classe $C^{1}$, com ponto fixo $x$.

a) Se todos os autovalores da matriz Jacobiana DT(x) têm módulo menor que 1, então o ponto fixo $x$ é assintoticamente estável.

b) Se pelo menos um dos autovalores de DT(x) tem módulo maior que 1, então x é instável. 


\subsection{Conjuntos $\alpha$-limite e $w$-limite de uma órbita.}

Sejam $X$ um subconjunto aberto do espaço euclidiano $\mathbb{R}^{n}$, e $T: X \longrightarrow \mathbb{R}^{n}$ um campo vetorial de classe $C^{k}, k \geq 1$. Seja $\varphi(t)=\varphi(t, x)$ a curva integral de $T$ passando pelo ponto $x$, definida no seu intervalo máximo $I_{x}=\left(w_{-}(x), w_{+}(x)\right)$. Se $w_{+}(x)=\infty$, define-se o conjunto

$$
w(x)=\left\{q \in X ; \exists\left\{t_{n}\right\} \operatorname{com}_{n} \longrightarrow \infty e \varphi\left(t_{n}\right) \longrightarrow q, \text { quando } n \longrightarrow \infty\right\}
$$

Analogamente, se $w_{-}(x)=-\infty$, define-se o conjunto

$$
\alpha(x)=\left\{q \in X ; \exists\left\{t_{n}\right\} \operatorname{com}_{n} \longrightarrow-\infty e \varphi\left(t_{n}\right) \longrightarrow q \text {, quando } n \longrightarrow \infty\right\}
$$

Os conjuntos $w(x)$ e $\alpha(x)$ são chamados, respectivamente, de conjunto $w$-limite e conjunto $\alpha$-limite de $x$.

Definição 1.4 O conjunto w-limite de uma órbita $\gamma$, que denotaremos por $w(\gamma)$, é o conjunto $w(x)$, para qualquer $x \in \gamma$. O conjunto $\alpha$-limite de uma órbita $\gamma$, que denotaremos por $\alpha(\gamma)$, é o conjunto $\alpha(x)$, para qualquer $x \in \gamma$. E $w(x)=w(y)$ se $x, y \in \gamma$.

Observação 1.1 Sejam $\varphi(t)=\varphi(t, x)$ a curva integral do campo T pelo ponto $x$ e $\psi(t)=\psi(t, x)$ a curva integral do campo $-T$ pelo ponto $x$, então $\psi(t, x)=\varphi(-t, x)$. Segue-se daí que o w-limite de $\psi(t)$ é igual ao $\alpha$-limite de $\varphi(t)$ e, reciprocamente, o w-limite de $\varphi(t)$ é igual ao $\alpha$-limite de $\psi(t)$. Por este motivo, para estudarmos as propriedades gerais dos conjuntos $\alpha$-limite e $w$-limite de órbitas é suficiente nos restringirmos ao estudo do conjunto w-limite.

Definição 1.5 Um ponto $x$ é chamado de positivamente recorrente se $x \in w(x)$, e se $T$ é inversível, $x$ é negativamente recorrente se $x \in \alpha(x)$. x é dito recorrente se é positivamente e negativamente recorrente.

Exemplo 1.2 Pontos periódicos são pontos recorrentes.

Observação 1.2 O conjunto $\mathcal{R}(T)$ dos pontos recorrentes é T-invariante.

\subsection{O Teorema da Bifurcação de Hopf em $\mathbb{R}^{2}$ e $\mathbb{R}^{n}$.}

Teorema 1.3 (da bifurcação de Hopf em $\mathbb{R}^{2}$ ) Seja $\Psi_{\mu}$ um campo de vetores de classe $C^{k}(k \geq 4)$ em $\mathbb{R}^{2}$, tal que $\Psi_{\mu}(0)=0$ para todo $\mu$ e $\Psi=\left(\Psi_{\mu}, 0\right)$ é também $C^{k}$. Suponhamos que $D \Psi_{\mu}(0,0)$ tem dois autovalores 
complexos conjugados distintos $\lambda(\mu)$ e $\overline{\lambda(\mu)}$, tais que, para $\mu>0$ temos que $\operatorname{Re} \lambda(\mu)>0$.Suponhamos também que $\frac{d(\operatorname{Re} \lambda(\mu))}{d \mu}>0$ em $\mu=0$. Então:

a) Existe uma função $\mu:(-\epsilon, \epsilon) \rightarrow \mathbb{R}$ de classe $C^{k-2}$, tal que $\mu(0)=0$ e para todo $x_{1} \neq 0,\left(x_{1}, 0, \mu\left(x_{1}\right)\right)$ está sobre uma órbita periódica fechada de $\Psi$ com raio crescente da ordem de $\sqrt{\mu}$ e período próximo de $\frac{2 \pi}{|\lambda(0)|}$.

b) Existe uma vizinhança $U$ de $(0,0,0) \mathrm{em} \mathbb{R}^{3}$ tal que qualquer órbita fechada em $U$ é uma dessas acima. Além disso, se $(0,0)$ é um atrator "indefinido"para $\Psi_{0}$, então:

c) $\mu\left(x_{1}\right)>0$ para todo $x_{1} \neq 0$ e as órbitas periódicas são atratoras.

Definição $1.6(0,0)$ é um atrator "indefinido"para $\Psi_{0}$ se $\frac{\partial^{3} V}{\partial x_{1}^{3}}(0,0)<0$. Onde $V\left(x_{1}, \mu\right)=P\left(x_{1}, \mu\right)-x_{1}$ e P é a transformação do primeiro retorno do campo $\Psi$ em relação ao eixo $\left(x_{1}, 0\right)$.

Teorema 1.4 (da bifurcação de Hopf em $\mathbb{R}^{n}$ ) Seja $\Psi_{\mu}$ um campo de vetores em $\mathbb{R}^{n}$ de classe $C^{k+1}$, com $k \geq 4$, com todas as hipóteses do Teorema 1.3 mantidas exceto que assumimos que o resto do espectro é distinto dos dois autovalores simples $\lambda(\mu), \overline{\lambda(\mu)}$. Então a conclusão a) é verdadeira. A conclusão b) é verdadeira se o resto do espectro permanece do lado direito do semi plano quando $\mu$ passa pelo 0 . A conclusão $c)$ é verdade se, em relação a $\lambda(\mu), \overline{\lambda(\mu)}, 0$ é um atrator "indefinido"no mesmo sentido do Teorema 1.3. e se quando as coordenadas são escolhidas de modo que

$$
D \Psi_{0}(0)=\left(\begin{array}{ccc}
0 & |\lambda(0)| & D_{3} \Psi^{1}(0) \\
-|\lambda(0)| & 0 & D_{3} \Psi^{2}(0) \\
0 & 0 & D_{3} \Psi^{3}(0)
\end{array}\right),
$$

$\lambda(0) \notin \sigma\left(D_{3} \Psi^{3}(0)\right)$ (espectro de $D_{3} \Psi^{2}(0)$ ).

As demonstrações dos Teoremas 1.3 e 1.4 não serão feitas neste trabalho, mas podem ser vistas em [5].

\subsection{Variedade Central.}

Nesta seção enunciaremos o Teorema da Variedade Central. Esse teorema será importante pois nos permitirá reduzir o estudo de uma variedade de dimensão finita qualquer para uma outra de 
dimensão 2. Para os leitores interessados é possível ver a demonstração do mesmo no apêndice $\mathrm{B}$ do artigo [1]. Antes de enunciarmos o teorema, precisamos de alguns resultados de Álgebra Linear, que seguem adiante.

Definição 1.7 Seja B uma matriz complexa $n \times n$. Se $\lambda$ é um autovalor de B, seja

$$
V_{\lambda}=\left\{v \in \mathbb{C}^{n}:(B-\lambda I)^{i} v=0 \text { paraalgum } i \in \mathbb{N}\right\} .
$$

Se Bé real e $\gamma$ é um autovalor real, seja

$$
V_{\gamma}^{\mathbb{R}}=\mathbb{R}^{n} \cap V_{\gamma}=\left\{v \in \mathbb{R}^{n}:(B-\gamma I)^{i} v=0 \text { paraalgumi } \in \mathbb{N}\right\}
$$

Se B é real e $\lambda, \bar{\lambda}$ é um par de autovalores complexos, seja

$$
V_{\lambda, \bar{\lambda}}^{\mathbb{R}}=\mathbb{R}^{n} \cap\left(V_{\lambda} \oplus V_{\bar{\lambda}}\right)
$$

Esses espaços são chamados de autoespaços generalizados.

Teorema 1.5 (da Variedade Central) Seja $\Psi$ uma aplicação da vizinhança do zero no espaço de Banach z em Z. Suponhamos que $\Psi$ tem $k+1$ derivadas contínuas, que $\Psi(0)=0, D \Psi(0)$ tem raio espectral 1 e que o espectro de $D \Psi(0)$ tem uma parte contida na circunferência unitária com um número finito de autovalores, e o restante está a uma distância não nula da mesma. Suponhamos que $Y$, o auto-espaço generalizado de $D \Psi(0)$ pertencente à parte do espectro sobre a circunferência unitária, tem dimensão $d<\infty$. Então existe uma vizinhança $\mathcal{V}$ do 0 em $z$ e uma subvariedade $\mathcal{M} \subset \mathcal{V}$ de classe $C^{k}$ e dimensão $d$, contendo a origem $e$ tangente a $Y$ em 0 , tal que:

a) (Invariância Local) Se $x \in \mathcal{M} e \Psi(x) \in \mathcal{V}$, então $\Psi(x) \in \mathcal{M}$.

b) (Atratividade Local) Se $\Psi^{n}(x) \in \mathcal{V}$ para todo $n \in \mathbb{N}$, então quando $n \rightarrow \infty$, a distância de $\Psi^{n}(x)$ a $\mathcal{M}$ vai a zero.

Uma variedade $\mathcal{M}$ como descrita no teorema é chamada de variedade central (para o ponto fixo 0 de $\Psi$ ). Não é em geral única, mas por $b$ ), pelo menos contém todos os pontos $w$-limite de uma órbita $\left\{\Psi^{n}(x) / n=0,1,2, \ldots\right\}$ que está contida em uma vizinhança do zero. 


\section{CAPÍTULO 2}

\section{Bifurcação de Poincaré-Andronov-Hopf para Difeomorfismos do Plano.}

Neste capítulo trabalharemos com um difeomorfismo $\Phi_{\mu}$. A utilização do Teorema 1.5 nos permitirá reduzir o estudo ao plano. Apresentaremos uma forma canônica para o difeomorfismo e daremos condições para a existência de bifurcação. Posteriormente mostraremos a existência de uma curva fechada invariante para a aplicação $\Phi_{\mu}$, utilizando o princípio da contração.

\subsection{Difeomorfismos do Plano.}

Uma das técnicas utilizadas por Ruelle e Takens é a redução de um problema geral para outro em dimensão 2. Eles fizeram isto utilizando o Teorema 1.5, mas a idéia aqui é aplicá-lo para a transformação:

$$
\Psi:(x, \mu) \longmapsto\left(\Phi_{\mu}(x), \mu\right),
$$

onde $\Phi_{\mu}$ é um difeomorfismo do espaço de Banach $z$ em $z$, que depende de um parâmetro real $\mu$. Se $D \Phi_{0}$ tem dois autovalores complexos conjugados simples na circunferência unitária, então o auto-espaço generalizado de $D \Phi(0)$ tem dimensão 3. A dimensão extra encontra-se na direção de $\mu$. O Teorema 1.5 então garante a existência de uma variedade central $\mathcal{M}$ de dimensão 3 . Se 
fixarmos $\mu$ suficientemente pequeno, obteremos uma seção $\mathcal{M}_{\mu}$ de dimensão 2 de $\mathcal{M}$, que é localmente invariante e atratora para $\Phi_{\mu}$. Como estamos interessados no comportamento recorrente, podemos restringir $\Phi_{\mu}$ a $\mathcal{M}_{\mu}$. Estamos então reduzindo o estudo a uma família de transformações suficientemente regulares ( $C^{4}$ é suficiente) a um parâmetro real, que denotaremos ainda por $\Phi_{\mu}$, de uma vizinhança do zero em $\mathbb{R}^{2}$ sobre $\mathbb{R}^{2}$, com $\Phi_{\mu}(0,0)=(0,0)$ e $D \Phi_{0}(0,0)$ tendo dois autovalores complexos conjugados distintos, sobre a circunferência unitária.

\subsection{Forma Canônica da Aplicação $\Phi_{\mu}$.}

Queremos mostrar a existência de uma curva fechada invariante para $\Phi_{\mu}$, com $\mu>0$. Para isto é importante encontrar uma forma conveniente para $\Phi_{\mu}$. Assumimos que os autovalores de $D \Phi_{\mu}(0,0)$ passam através da circunferência unitária com velocidade positiva quando $\mu$ passa pelo 0 . Podemos então reparametrizar os autovalores de $D \Phi_{\mu}(0,0)$ de forma a obtê-los como $(1+\mu) e^{i \theta(\mu)}$ e $(1+\mu) e^{-i \theta(\mu)}$, o que muda a velocidade, mas não os valores para $\mu=0$. Fazendo uma mudança de coordenadas suave $\mu$-dependente, podemos denotar $D \Phi_{\mu}(0,0)$ por:

$$
D \Phi_{\mu}(0,0)=(1+\mu)\left(\begin{array}{rr}
\cos \theta(\mu) & -\operatorname{sen} \theta(\mu) \\
\operatorname{sen} \theta(\mu) & \cos \theta(\mu)
\end{array}\right) \text {. }
$$

Assim, a aplicação $\Phi_{\mu}$ pode ser denotada por:

$$
\begin{aligned}
\Phi_{\mu}: \mathbb{R}^{2} & \longrightarrow \mathbb{R}^{2} \\
\left(x_{1}, x_{2}\right) & \longmapsto(1+\mu)\left(\begin{array}{rr}
\cos \theta(\mu) & -\operatorname{sen} \theta(\mu) \\
\operatorname{sen} \theta(\mu) & \cos \theta(\mu)
\end{array}\right)\left(\begin{array}{l}
x_{1} \\
x_{2}
\end{array}\right)+g_{\mu}\left(x_{1}, x_{2}\right),
\end{aligned}
$$

onde $g_{\mu}$ é uma função suave com componentes $g_{1}$ e $g_{2}$, tendo expansão em série de Taylor iniciando-se com termos quadráticos, ou seja, $g_{\mu}=O\left(\left\|\left(x_{1}, x_{2}\right)\right\|^{2}\right)$ uniformemente em $\mu$. Temos que $\left|g_{\mu}\right|$ é menor do que um polinômio complexo com coeficientes contínuos em $\mu$, com termos de ordem maior ou igual a 2. O próximo passo é fazer mais uma mudança de coordenada para levar $\Phi_{\mu}$ a uma forma canônica apropriada. A aplicação $\Phi_{\mu}$ pode ser escrita na forma:

$$
\Phi_{\mu}\left(x_{1}, x_{2}\right)=(1+\mu)\left(x_{1} \cos \theta(\mu)-x_{2} \operatorname{sen} \theta(\mu), x_{1} \operatorname{sen} \theta(\mu)+x_{2} \cos \theta(\mu)\right)+g_{\mu}\left(x_{1}, x_{2}\right) \text {. }
$$


Vamos escrever cada par $\left(x_{1}, x_{2}\right) \in \mathbb{R}^{2}$ como um número complexo $z=x_{1}+i x_{2}$ e, tomando $\widetilde{\Phi}_{\mu}(z)=\Phi_{1}\left(x_{1}, x_{2}\right)+i \Phi_{2}\left(x_{1}, x_{2}\right)$, onde $\Phi_{\mu}=\left(\Phi_{1}, \Phi_{2}\right)$, teremos:

$$
\begin{aligned}
\widetilde{\Phi}_{\mu}(z) & =(1+\mu)\left[x_{1} \cos \theta(\mu)-x_{2} \operatorname{sen} \theta(\mu)\right]+i(1+\mu)\left[x_{1} \operatorname{sen} \theta(\mu)+x_{2} \cos \theta(\mu)\right]+O\left(|z|^{2}\right) \\
& =(1+\mu)[\cos \theta(\mu)+i \operatorname{sen} \theta(\mu)]\left(x_{1}+i x_{2}\right)+O\left(|z|^{2}\right) \\
& =(1+\mu) e^{i \theta(\mu)} z+O\left(|z|^{2}\right) .
\end{aligned}
$$

Tomando $\lambda(\mu)=(1+\mu) e^{i \theta(\mu)}$ a equação acima toma a forma:

$$
\widetilde{\Phi}_{\mu}(z)=\lambda(\mu) z+O\left(|z|^{2}\right) .
$$

Ao longo desse texto será comum aparecer a expressão $O\left(|z|^{n}\right) \operatorname{com} n \in \mathbb{N}$. Com isto queremos dizer que $\frac{\left|\widetilde{\Phi}_{\mu}(z)-\lambda(\mu) z\right|}{|z|^{n}} \leq K$, com $K$ uma constante independente de $\mu$, ou seja, a aproximação é uniforme em $\mu$. De agora em diante, também para facilitar a notação, denotaremos $\widetilde{\Phi}_{\mu}$ por $\Phi_{\mu}$.

Lema 2.1 A aplicação $\Phi_{\mu}(z)$ pode ser escrita em série de Taylor centrada na origem, na forma:

$$
\Phi_{\mu}(z)=\lambda(\mu) z+A_{2}(z)+A_{3}(z)+O\left(|z|^{4}\right)
$$

onde

$$
A_{k}(z)=\sum_{j=0}^{k} \xi_{j(k-j)} z^{j}(\bar{z})^{k-j},
$$

para $k=2 e 3$ e $\xi_{j(k-j)} \in \mathbb{C}$. Em particular,

$$
\begin{aligned}
& \xi_{20}=\frac{1}{8}\left\{\left(g_{1}\right)_{x_{1} x_{1}}-\left(g_{1}\right)_{x_{2} x_{2}}+2\left(g_{2}\right)_{x_{1} x_{2}}+i\left[\left(g_{2}\right)_{x_{1} x_{1}}-\left(g_{2}\right)_{x_{2} x_{2}}-2\left(g_{1}\right)_{x_{1} x_{2}}\right]\right\}, \\
& \xi_{11}=\frac{1}{4}\left\{(g 1)_{x_{1} x_{1}}+\left(g_{1}\right)_{x_{2} x_{2}}+i\left[\left(g_{2}\right)_{x_{1} x_{1}}+\left(g_{2}\right)_{x_{2} x_{2}}\right]\right\}, \\
& \xi_{02}=\frac{1}{8}\left\{\left(g_{1}\right)_{x_{1} x_{1}}-\left(g_{1}\right)_{x_{2} x_{2}}-2\left(g_{2}\right)_{x_{1} x_{2}}+i\left[\left(g_{2}\right)_{x_{1} x_{1}}-\left(g_{2}\right)_{x_{2} x_{2}}+2\left(g_{1}\right)_{x_{1} x_{2}}\right]\right\} e \\
& \xi_{21}=\frac{1}{16}\left\{\left(g_{1}\right)_{x_{1} x_{1} x_{1}}+\left(g_{1}\right)_{x_{1} x_{2} x_{2}}+\left(g_{2}\right)_{x_{1} x_{1} x_{2}}+\left(g_{2}\right)_{x_{2} x_{2} x_{2}}+i\left[\left(g_{2}\right)_{x_{1} x_{1} x_{1}}+\left(g_{2}\right)_{x_{1} x_{2} x_{2}}-\left(g_{1}\right)_{x_{1} x_{1} x_{2}}-\left(g_{1}\right)_{x_{2} x_{2} x_{2}}\right]\right\} .
\end{aligned}
$$

Demonstração: Vamos reescrever os termos de ordem maior ou igual a 2 de $\Phi_{\mu}(z)$. Já sabemos que $g_{\mu}$ tem componentes $g_{1}$ e $g_{2}$ de ordem 2 e, para facilitar a notação, omitiremos a dependência de 
$g_{1}$ e $g_{2}$ em $\mu$. A série de Taylor de $g$ na origem é dada por:

$$
\begin{aligned}
g_{1}\left(x_{1}, x_{2}\right)= & \frac{1}{2}\left[\left(g_{1}\right)_{x_{1} x_{1}} x_{1}^{2}+2\left(g_{1}\right)_{x_{1} x_{2}} x_{1} x_{2}+\left(g_{1}\right)_{x_{2} x_{2}} x_{2}^{2}\right]+ \\
& \frac{1}{3 !}\left[\left(g_{1}\right)_{x_{1} x_{1} x_{1}} x_{1}^{3}+3\left(g_{1}\right)_{x_{1} x_{1} x_{2}} x_{1}^{2} x_{2}+3\left(g_{1}\right)_{x_{1} x_{2} x_{2}} x_{1} x_{2}^{2}+\left(g_{1}\right)_{x_{2} x_{2} x_{2}} x_{2}^{3}\right]+O\left(\left\|\left(x_{1}, x_{2}\right)\right\|^{2}\right) .
\end{aligned}
$$

Analogamente obtemos a série de Taylor para $g_{2}$. Tomando $g_{\mu}(z)=g_{\mu}\left(x_{1}+i x_{2}\right)=g_{1}\left(x_{1}, x_{2}\right)+$ $i g_{2}\left(x_{1}, x_{2}\right)$, teremos:

$$
\begin{aligned}
g_{\mu}\left(x_{1}+i x_{2}\right)= & \frac{1}{2}\left[\left(g_{1}\right)_{x_{1} x_{1}} x_{1}^{2}+2\left(g_{1}\right)_{x_{1} x_{2}} x_{1} x_{2}+\left(g_{1}\right)_{x_{2} x_{2}} x_{2}^{2}\right]+ \\
& \frac{1}{3 !}\left[\left(g_{1}\right)_{x_{1} x_{1} x_{1}} x_{1}^{3}+3\left(g_{1}\right)_{x_{1} x_{1} x_{2}} x_{1}^{2} x_{2}+3\left(g_{1}\right)_{x_{1} x_{2} x_{2}} x_{1} x_{2}^{2}+\left(g_{1}\right)_{x_{2} x_{2} x_{2}} x_{2}^{3}\right]+ \\
& \frac{i}{2}\left[\left(g_{2}\right)_{x_{1} x_{1}} x_{1}^{2}+2\left(g_{2}\right)_{x_{1} x_{2}} x_{1} x_{2}+\left(g_{2}\right)_{x_{2} x_{2}} x_{2}^{2}\right]+ \\
& \frac{i}{3 !}\left[\left(g_{2}\right)_{x_{1} x_{1} x_{1}} x_{1}^{3}+3\left(g_{2}\right)_{x_{1} x_{1} x_{2}} x_{1}^{2} x_{2}+3\left(g_{2}\right)_{x_{1} x_{2} x_{2}} x_{1} x_{2}^{2}+\left(g_{2}\right)_{x_{2} x_{2} x_{2}} x_{2}^{3}\right]+O\left(\left\|\left(x_{1}, x_{2}\right)\right\|^{2}\right) .
\end{aligned}
$$

Agora, tomando $x_{1}=\frac{z+\bar{z}}{2}$ e $x_{2}=\frac{z-\bar{z}}{2 i}$ na última expressão, e organizando os termos, chegamos a:

$$
\begin{aligned}
g_{\mu}(z)= & \frac{1}{2}\left[\frac{\left(g_{1}\right)_{x_{1} x_{1}}}{4}-\frac{i\left(g_{1}\right)_{x_{1} x_{2}}}{2}-\frac{\left(g_{1}\right)_{x_{2} x_{2}}}{4}+\frac{i\left(g_{2}\right)_{x_{1} x_{1}}}{4}+\frac{\left(g_{2}\right)_{x_{1} x_{2}}}{2}-\frac{i\left(g_{2}\right)_{x_{2} x_{2}}}{4}\right] z^{2}+ \\
& \frac{1}{2}\left[\frac{\left(g_{1}\right)_{x_{1} x_{1}}}{2}+\frac{\left(g_{1}\right)_{x_{2} x_{2}}}{2}+\frac{i\left(g_{2}\right)_{x_{1} x_{1}}}{2}+\frac{i\left(g_{2}\right)_{x_{2} x_{2}}}{2}\right] z \bar{z}+ \\
& \frac{1}{2}\left[\frac{\left(g_{1}\right)_{x_{1} x_{1}}}{4}+\frac{i\left(g_{1}\right)_{x_{1} x_{2}}}{2}-\frac{\left(g_{1}\right)_{x_{2} x_{2}}}{4}+\frac{i\left(g_{2}\right)_{x_{1} x_{1}}}{4}+\frac{\left(g_{2}\right)_{x_{1} x_{2}}}{2}-\frac{\left.i\left(g_{2}\right)_{x_{2} x_{2}}\right]}{4}\right] \bar{z}^{2}+ \\
& \xi_{30} z^{3}+\frac{1}{3 !}\left[\frac{3\left(g_{1}\right)_{x_{1} x_{1} x_{1}}}{8}-\frac{3 i\left(g_{1}\right)_{x_{1} x_{1} x_{2}}}{8}+\frac{3\left(g_{1}\right)_{x_{1} x_{2} x_{2}}}{8}-\frac{3 i\left(g_{1}\right)_{x_{2} x_{2} x_{2}}}{8}+\frac{3 i\left(g_{2}\right)_{x_{1} x_{1} x_{1}}}{8}+\right. \\
& \left.\frac{3\left(g_{2}\right)_{x_{1} x_{1} x_{2}}}{8}+\frac{3 i\left(g_{2}\right)_{x_{1} x_{2} x_{2}}}{8}+\frac{3\left(g_{2}\right)_{x_{2} x_{2} x_{2}}}{8}\right] z^{2} \bar{z}+\xi_{12} z \bar{z}^{2}+\xi_{03} \bar{z}^{3}+O\left(|z|^{4}\right) .
\end{aligned}
$$

Vale a pena observar que, no desenvolvimento de um termo de grau $n$ nas variáveis $x_{1}, x_{2}$ só aparecem termos de grau $n$ em $(z, \bar{z})$. Assim é possível escrever $\Phi_{\mu}(z)$ na forma:

$$
\Phi_{\mu}(z)=\lambda(\mu) z+A_{2}(z)+A_{3}(z)+O\left(|z|^{4}\right)
$$


onde $A_{k}(z)=\sum_{j=0}^{k} \xi_{j(k-j)} z^{j}(\bar{z})^{k-j}$, para $k=2 e 3 \quad$ e $\xi_{j(k-j)} \in \mathbb{C}$. Em particular, da equação (2.2) obtemos:

$$
\begin{gathered}
\xi_{20}=\frac{1}{8}\left\{\left(g_{1}\right)_{x_{1} x_{1}}-\left(g_{1}\right)_{x_{2} x_{2}}+2\left(g_{2}\right)_{x_{1} x_{2}}+i\left[\left(g_{2}\right)_{x_{1} x_{1}}-\left(g_{2}\right)_{x_{2} x_{2}}-2\left(g_{1}\right)_{x_{1} x_{2}}\right]\right\} \\
\xi_{11}=\frac{1}{4}\left\{\left(g_{1}\right)_{x_{1} x_{1}}+\left(g_{1}\right)_{x_{2} x_{2}}+i\left[\left(g_{2}\right)_{x_{1} x_{1}}+\left(g_{2}\right)_{x_{2} x_{2}}\right]\right\} \\
\xi_{02}=\frac{1}{8}\left\{\left(g_{1}\right)_{x_{1} x_{1}}-\left(g_{1}\right)_{x_{2} x_{2}}-2\left(g_{2}\right)_{x_{1} x_{2}}+i\left[\left(g_{2}\right)_{x_{1} x_{1}}-\left(g_{2}\right)_{x_{2} x_{2}}+2\left(g_{1}\right)_{x_{1} x_{2}}\right]\right\} \mathrm{e}
\end{gathered}
$$

$\xi_{21}=\frac{1}{16}\left\{\left(g_{1}\right)_{x_{1} x_{1} x_{1}}+\left(g_{1}\right)_{x_{1} x_{2} x_{2}}+\left(g_{2}\right)_{x_{1} x_{1} x_{2}}+\left(g_{2}\right)_{x_{2} x_{2} x_{2}}+i\left[\left(g_{2}\right)_{x_{1} x_{1} x_{1}}+\left(g_{2}\right)_{x_{1} x_{2} x_{2}}-\left(g_{1}\right)_{x_{1} x_{1} x_{2}}-\left(g_{1}\right)_{x_{2} x_{2} x_{2}}\right]\right\}$.

Nosso objetivo agora é, através de mudanças de coordenadas para a aplicação (2.3), eliminar os termos de ordem 2, 3 e 4 da expressão de $\Phi_{\mu}$.

Lema 2.2 Seja

$$
\Phi_{\mu}(z)=\lambda z+A_{2}(z)+A_{3}(z)+O\left(|z|^{4}\right)
$$

a aplicação definida em (2.3), com coeficientes em (2.4) e $\lambda=\lambda(\mu)=(1+\mu) e^{i \theta(\mu)}$. Suponhamos que $e^{i \theta(0)} \neq 1$ e $e^{3 i \theta(0)} \neq 1$. Então $\Phi_{\mu}$ pode ser transformada pela mudança de coordenadas complexa

$$
\begin{aligned}
\tau: \mathbb{C} & \longrightarrow \mathbb{C} \\
z & \longmapsto z^{\prime}=z+\gamma(z),
\end{aligned}
$$

onde $\gamma(z)=\gamma_{\mu}(z)=\gamma_{2} z^{2}+\gamma_{1} z \bar{z}+\gamma_{0} \bar{z}^{2}, \operatorname{com} \gamma_{2}=\frac{\xi_{20}}{\lambda-\lambda^{2}}, \gamma_{1}=\frac{\xi_{11}}{\lambda-|\lambda|^{2}} e \gamma_{0}=\frac{\xi_{02}}{\lambda-\bar{\lambda}^{2}}$, na aplicação sem termos quadráticos:

$$
\widetilde{\Phi}_{\mu}\left(z^{\prime}\right)=\lambda z^{\prime}+B_{3}\left(z^{\prime}\right)+O\left(\left|z^{\prime}\right|^{4}\right)
$$

$\operatorname{com} B_{3}\left(z^{\prime}\right)=\sum_{j=0}^{3} \alpha_{j}^{3} z^{\prime j}\left(\overline{z^{\prime}}\right)^{3-j}$ e $\alpha_{j}^{3} \in \mathbb{C}$

Demonstração: Temos que, em uma vizinhança da origem no novo sistema de coordenadas $z^{\prime}, \overline{z^{\prime}}$, é possível inverter a aplicação $\tau$, obtendo:

$$
z=z^{\prime}+\gamma^{\prime}\left(z^{\prime}\right)+k\left(z^{\prime}\right)+O\left(\left|z^{\prime}\right|^{4}\right)
$$


onde grau $\gamma^{\prime}=2$ e grau $k=3$. Da expressão de $\tau$ e da equação (2.5) teremos:

$$
\gamma^{\prime}\left(z^{\prime}\right)+k\left(z^{\prime}\right)+\gamma\left(z^{\prime}+\gamma^{\prime}\left(z^{\prime}\right)+k\left(z^{\prime}\right)+O\left(\left|z^{\prime}\right|^{4}\right)\right)+O\left(\left|z^{\prime}\right|^{4}\right)=0
$$

Vamos calcular $\gamma\left(z^{\prime}+\gamma^{\prime}\left(z^{\prime}\right)+k\left(z^{\prime}\right)+O\left(\left|z^{\prime}\right|^{4}\right)\right)$. Agrupando os termos de mesma ordem e usando a definição de $\gamma(z)$, obtemos:

$$
\begin{aligned}
\gamma\left(z^{\prime}+\gamma^{\prime}\left(z^{\prime}\right)+k\left(z^{\prime}\right)+O\left(\left|z^{\prime}\right|^{4}\right)\right)= & \gamma_{2}\left[z^{\prime}+\gamma^{\prime}\left(z^{\prime}\right)+k\left(z^{\prime}\right)+O\left(\left|z^{\prime}\right|^{4}\right)\right]^{2}+ \\
& \gamma_{1}\left[z^{\prime}+\gamma^{\prime}\left(z^{\prime}\right)+k\left(z^{\prime}\right)+O\left(\left|z^{\prime}\right|^{4}\right)\right]\left[\overline{z^{\prime}}+\overline{\gamma^{\prime}\left(z^{\prime}\right)}+\overline{k\left(z^{\prime}\right)}+O\left(\left|\overline{z^{\prime}}\right|^{4}\right)\right]+ \\
& \gamma_{0}\left[\overline{z^{\prime}}+\overline{\gamma^{\prime}\left(z^{\prime}\right)}+\overline{k\left(z^{\prime}\right)}+O\left(\left|\overline{z^{\prime}}\right|^{4}\right)\right]^{2} \\
= & \gamma_{2}\left[z^{\prime 2}+2 z^{\prime} \gamma^{\prime}\left(z^{\prime}\right)\right]+\gamma_{1}\left[z^{\prime} \overline{z^{\prime}}+z^{\prime} \overline{\gamma^{\prime}\left(z^{\prime}\right)}+\overline{z^{\prime}} \gamma^{\prime}\left(z^{\prime}\right)\right]+ \\
& \gamma_{0}\left[\overline{z^{\prime}}+2 \overline{z^{\prime} \gamma^{\prime}\left(z^{\prime}\right)}\right]+O\left(\left|z^{\prime}\right|^{4}\right) \\
= & \gamma\left(z^{\prime}\right)+\gamma_{2}\left[2 z^{\prime} \gamma^{\prime}\left(z^{\prime}\right)\right]+\gamma_{1}\left[z^{\prime} \overline{\gamma^{\prime}\left(z^{\prime}\right)}+\overline{z^{\prime}} \gamma^{\prime}\left(z^{\prime}\right)\right]+ \\
& \gamma_{0}\left[2 \overline{z^{\prime} \gamma^{\prime}\left(z^{\prime}\right)}\right]+O\left(\left|z^{\prime}\right|^{4}\right) .
\end{aligned}
$$

Substituindo (2.7) em (2.6), chegamos a:

$$
\gamma^{\prime}\left(z^{\prime}\right)+\gamma\left(z^{\prime}\right)+k\left(z^{\prime}\right)+\gamma_{2}\left[2 z^{\prime} \gamma^{\prime}\left(z^{\prime}\right)\right]+\gamma_{1}\left[z^{\prime} \overline{\gamma^{\prime}\left(z^{\prime}\right)}+\overline{z^{\prime}} \gamma^{\prime}\left(z^{\prime}\right)\right]+\gamma_{0}\left[2 \overline{z^{\prime} \gamma^{\prime}\left(z^{\prime}\right)}\right]+O\left(\left|z^{\prime}\right|^{4}\right)=0 .
$$

Comparando termos de mesma ordem, concluímos que:

$$
\gamma^{\prime}\left(z^{\prime}\right)=-\gamma\left(z^{\prime}\right) \quad \text { e } \quad k\left(z^{\prime}\right)=\gamma_{2}\left[2 z^{\prime} \gamma\left(z^{\prime}\right)\right]+\gamma_{1}\left[z^{\prime} \overline{\gamma\left(z^{\prime}\right)}+\overline{z^{\prime}} \gamma\left(z^{\prime}\right)\right]+\gamma_{0}\left[2 \overline{z^{\prime} \gamma\left(z^{\prime}\right)}\right]
$$

Denotando $\widetilde{\Phi}_{\mu}$ como sendo $\Phi_{\mu}$ na nova coordenada $z^{\prime}$, teremos :

$$
\begin{aligned}
\widetilde{\Phi}_{\mu}\left(z^{\prime}\right) & =\left(\tau \circ \Phi_{\mu} \circ \tau^{-1}\right)\left(z^{\prime}\right) \\
& =\left(\tau \circ \Phi_{\mu}\right)(z) \\
& =\Phi_{\mu}(z)+\gamma\left(\Phi_{\mu}(z)\right) \\
& =\Phi_{\mu}\left(z^{\prime}-\gamma\left(z^{\prime}\right)+k\left(z^{\prime}\right)+O\left(\left|z^{\prime}\right|^{4}\right)\right)+\gamma\left(\Phi_{\mu}\left(z^{\prime}-\gamma\left(z^{\prime}\right)+k\left(z^{\prime}\right)+O\left(\left|z^{\prime}\right|^{4}\right)\right)\right)
\end{aligned}
$$


Vamos desenvolver a equação acima. Agrupando os termos de mesma ordem de $\Phi_{\mu}\left(z^{\prime}-\gamma\left(z^{\prime}\right)+\right.$ $\left.k\left(z^{\prime}\right)+O\left(\left|z^{\prime}\right|^{4}\right)\right)$, segue que:

$$
\begin{aligned}
\Phi_{\mu}\left(z^{\prime}-\gamma\left(z^{\prime}\right)+k\left(z^{\prime}\right)+O\left(\left|z^{\prime}\right|^{4}\right)\right)= & \lambda z^{\prime}+\left\{-\lambda \gamma\left(z^{\prime}\right)+\xi_{20} z^{2}+\xi_{11} z^{\prime} \overline{z^{\prime}}+\xi_{02} \overline{z^{\prime}}{ }^{2}\right\}+ \\
& \left\{\lambda k\left(z^{\prime}\right)+\xi_{20}\left[-2 z^{\prime} \gamma\left(z^{\prime}\right)\right]+\xi_{11}\left[-z^{\prime} \overline{\gamma\left(z^{\prime}\right)}-\overline{z^{\prime}} \gamma\left(z^{\prime}\right)\right]+\right. \\
& \left.\xi_{02}\left[-2 \overline{z^{\prime} \gamma\left(z^{\prime}\right)}\right]+\xi_{30} z^{\prime 3}+\xi_{21} z^{\prime 2} \overline{z^{\prime}}+\xi_{12} \bar{z}^{\prime} \overline{z^{\prime}}+\xi_{03} \overline{z^{\prime}}\right\}+ \\
& O\left(\left|z^{\prime}\right|^{4}\right),
\end{aligned}
$$

e, pela definição de $\gamma(z)$, temos:

$$
\begin{aligned}
& \gamma\left(\Phi_{\mu}\left(z^{\prime}-\gamma\left(z^{\prime}\right)+k\left(z^{\prime}\right)+O\left(\left|z^{\prime}\right|^{4}\right)\right)\right)=\gamma\left(\lambda z^{\prime}+\left(-\lambda \gamma\left(z^{\prime}\right)+\xi_{20} z^{\prime 2}+\xi_{11} z^{\prime} \overline{z^{\prime}}+\xi_{02}{\overline{z^{\prime}}}^{2}\right)\right)+O\left(\left|z^{\prime}\right|^{4}\right) \\
& =\gamma_{2}\left\{\lambda z^{\prime}+\left[-\lambda \gamma\left(z^{\prime}\right)+\xi_{20} z^{2}+\xi_{11} z^{\prime} \overline{z^{\prime}}+\xi_{02}{\overline{z^{\prime}}}^{2}\right]\right\}^{2}+ \\
& \gamma_{1}\left\{\lambda z^{\prime}+\left[-\lambda \gamma\left(z^{\prime}\right)+\xi_{20} z^{\prime 2}+\xi_{11} z^{\prime} \overline{z^{\prime}}+\xi_{02} \bar{z}^{2}\right]\right\} \\
& \left\{\overline{\lambda z^{\prime}}+\left[-\overline{\lambda \gamma\left(z^{\prime}\right)}+\bar{\xi}_{20}{\overline{z^{\prime}}}^{2}+\bar{\xi}_{11} z^{\prime} \overline{z^{\prime}}+\bar{\xi}_{02} z^{\prime 2}\right]\right\}+ \\
& \gamma_{0}\left\{\overline{\lambda z^{\prime}}+\left[-\overline{\lambda \gamma\left(z^{\prime}\right)}+\bar{\xi}_{20}{\overline{z^{\prime}}}^{2}+\bar{\xi}_{11} z^{\prime}{\overline{z^{\prime}}}+\bar{\xi}_{02} z^{\prime 2}\right]\right\}^{2}+O\left(\left|z^{\prime}\right|^{4}\right) \\
& =\gamma\left(\lambda z^{\prime}\right)+\gamma_{2}\left\{2 \lambda z^{\prime}\left[-\lambda \gamma\left(z^{\prime}\right)+\xi_{20} z^{2}+\xi_{11} z^{\prime} \overline{z^{\prime}}+\xi_{02}{\overline{z^{\prime}}}^{2}\right]\right\}+ \\
& \gamma_{1}\left\{\lambda z^{\prime}\left(-\overline{\lambda \gamma\left(z^{\prime}\right)}+\bar{\xi}_{02} z^{\prime 2}+\bar{\xi}_{11} z^{\prime} \bar{z}^{\prime}+\bar{\xi}_{20}{\overline{z^{\prime}}}^{2}\right)+\right. \\
& \left.\overline{\lambda z^{\prime}}\left(-\lambda \gamma\left(z^{\prime}\right)+\xi_{20} z^{\prime 2}+\xi_{11} z^{\prime} \overline{z^{\prime}}+\xi_{02}{\overline{z^{\prime}}}^{2}\right)\right\}+ \\
& \gamma_{0}\left\{2 \overline{\lambda z^{\prime}}\left[-\overline{\lambda \gamma\left(z^{\prime}\right)}+\bar{\xi}_{02} z^{\prime 2}+\bar{\xi}_{11} z^{\prime} \overline{z^{\prime}}+\bar{\xi}_{20}{\overline{z^{\prime}}}^{2}\right]\right\}+O\left(\left|z^{\prime}\right|^{4}\right) \text {. }
\end{aligned}
$$

Usando as equações (2.8), (2.9) e (2.10) teremos:

$$
\begin{aligned}
\widetilde{\Phi}_{\mu}\left(z^{\prime}\right)= & \lambda z^{\prime}+\left\{-\lambda \gamma\left(z^{\prime}\right)+\xi_{20} z^{\prime 2}+\xi_{11} z^{\prime} \overline{z^{\prime}}+\xi_{02}{\overline{z^{\prime}}}^{2}\right\}+\gamma\left(\lambda z^{\prime}\right)+O\left(\left|z^{\prime}\right|^{3}\right) \\
= & \lambda z^{\prime}-\lambda \gamma_{2} z^{2}-\lambda \gamma_{1} z^{\prime} \overline{z^{\prime}}-\lambda \gamma_{0}{\overline{z^{\prime}}}^{2}+\xi_{20} z^{2}+\xi_{11} z^{\prime} \overline{z^{\prime}}+\xi_{02}{\overline{z^{\prime}}}^{2}+ \\
& \lambda^{2} \gamma_{2} z^{\prime 2}+\lambda \bar{\lambda} \gamma_{1} z^{\prime} \overline{z^{\prime}}+\bar{\lambda}^{2} \gamma_{0}{\overline{z^{\prime}}}^{2}+O\left(\left|z^{\prime}\right|^{3}\right) \\
= & \lambda z^{\prime}+\left[\left(-\lambda \gamma_{2}+\xi_{20}+\lambda^{2} \gamma_{2}\right) z^{\prime 2}+\left(-\lambda \gamma_{1}+\xi_{11}+|\lambda|^{2} \gamma_{1}\right) z^{\prime} \overline{z^{\prime}}+\right. \\
& \left.\left(-\lambda \gamma_{0}+\xi_{02}+\bar{\lambda}^{2} \gamma_{0}\right){\overline{z^{\prime}}}^{2}\right]+O\left(\left|z^{\prime}\right|^{3}\right) .
\end{aligned}
$$

Observemos que $-\lambda \gamma_{2}+\xi_{20}+\lambda^{2} \gamma_{2}\left(\right.$ coeficiente de $z^{\prime 2}$ ) se anula quando tomamos $\gamma_{2}=\frac{\xi_{20}}{\lambda-\lambda^{2}}$. O coeficiente de $z^{\prime} \overline{z^{\prime}},-\lambda \gamma_{1}+\xi_{11}+|\lambda|^{2} \gamma_{1}$, se anula quando tomamos $\gamma_{1}=\frac{\xi_{11}}{\lambda-|\lambda|^{2}}$. Além disso, 
$-\lambda \gamma_{0}+\xi_{02}+\bar{\lambda}^{2} \gamma_{0}$ (coeficiente de ${\overline{z^{\prime}}}^{2}$ ) se anula quando tomamos $\gamma_{0}=\frac{\xi_{02}}{\lambda-\bar{\lambda}^{2}}$. Notemos que os denominadores nas expressões de $\gamma_{2}, \gamma_{1}$ e $\gamma_{0}$ não se anulam, visto que $|\lambda|=1+\mu$, e não há problema para $\mu \neq 0$. A mudança de coordenadas utilizada está bem definida quando $\mu \longrightarrow 0$, pois:

$$
\begin{aligned}
\lambda-\lambda^{2} \neq 0 & \Longleftrightarrow e^{i \theta(0)} \neq 1, \\
\lambda-1 \neq 0 & \Longleftrightarrow e^{i \theta(0)} \neq 1, \\
\lambda-\bar{\lambda}^{2} \neq 0 & \Longleftrightarrow e^{3 i \theta(0)} \neq 1 .
\end{aligned}
$$

Portanto, utilizando a mudança de coordenadas $\mu$-dependente para a aplicação $\Phi_{\mu}$, anulamos os termos de ordem quadrática. Logo, substituindo os valores de $\gamma_{2}, \gamma_{1}$ e $\gamma_{0}$ na equação (2.11), obtemos a aplicacão sem termos quadráticos:

$$
\widetilde{\Phi}_{\mu}\left(z^{\prime}\right)=\lambda z^{\prime}+B_{3}\left(z^{\prime}\right)+O\left(\left|z^{\prime}\right|^{4}\right)
$$

$\operatorname{com} B_{3}\left(z^{\prime}\right)=\sum_{j=0}^{3} \alpha_{j}^{3} z^{\prime j}\left(\overline{z^{\prime}}\right)^{3-j}$ e $\alpha_{j}^{3} \in \mathbb{C}$.

\section{Lema 2.3 Seja}

$$
\Phi_{\mu}(z)=\lambda z+B_{3}(z)+O\left(|z|^{4}\right)
$$

a aplicação definida em (2.12) e com $\lambda=\lambda(\mu)=(1+\mu) e^{i \theta(\mu)}$. Suponhamos que $e^{2 i \theta(0)} \neq 1$ e $e^{4 i \theta(0)} \neq 1$. Então $\Phi_{\mu}$ pode ser transformada pela mudança de coordenadas complexa

$$
\begin{aligned}
\tau: \mathbb{C} & \longrightarrow \mathbb{C} \\
z & \longmapsto z^{\prime}=z+\gamma(z),
\end{aligned}
$$

onde $\gamma(z)=\gamma_{\mu}(z)=\gamma_{3} z^{3}+\gamma_{2} z^{2} \bar{z}+\gamma_{1} z \bar{z}^{2}+\gamma_{0} \bar{z}^{3}, \operatorname{com} \gamma_{3}=\frac{\alpha_{3}^{3}}{\lambda-\lambda^{3}}, \gamma_{2}=0, \gamma_{1}=\frac{\alpha_{1}^{3}}{\lambda-\bar{\lambda}|\lambda|^{2}} e \gamma_{0}=\frac{\alpha_{0}^{3}}{\lambda-\bar{\lambda}^{3}}$, na aplicação com somente um termo cúbico:

$$
\widetilde{\Phi}_{\mu}\left(z^{\prime}\right)=\lambda z^{\prime}+\alpha_{2}^{3} z^{\prime 2} \overline{z^{\prime}}+C_{4}\left(z^{\prime}\right)+O\left(\left|z^{\prime}\right|^{5}\right),
$$

$\operatorname{com} C_{4}\left(z^{\prime}\right)=\sum_{j=0}^{4} \beta_{j}^{4} z^{\prime j}\left(\overline{z^{\prime}}\right)^{4-j} e \beta_{j}^{4} \in \mathbb{C}$ 
Demonstração: Temos que, em uma vizinhança da origem no novo sistema de coordenadas $z^{\prime}, \overline{z^{\prime}}$, é possível inverter a aplicação $\tau$, obtendo:

$$
z=z^{\prime}-\gamma\left(z^{\prime}\right)+O\left(\left|z^{\prime}\right|^{4}\right)
$$

Denotando $\widetilde{\Phi}_{\mu}$ como sendo $\Phi_{\mu}$ na nova coordenada $z^{\prime}$, teremos :

$$
\begin{aligned}
\widetilde{\Phi}_{\mu}\left(z^{\prime}\right) & =\left(\tau \circ \Phi_{\mu} \circ \tau^{-1}\right)\left(z^{\prime}\right) \\
& =\left(\tau \circ \Phi_{\mu}\right)(z) \\
& =\Phi_{\mu}(z)+\gamma\left(\Phi_{\mu}(z)\right) \\
& =\Phi_{\mu}\left(z^{\prime}-\gamma\left(z^{\prime}\right)+O\left(\left|z^{\prime}\right|^{4}\right)\right)+\gamma\left(\Phi_{\mu}\left(z^{\prime}-\gamma\left(z^{\prime}\right)+O\left(\left|z^{\prime}\right|^{4}\right)\right)\right) .
\end{aligned}
$$

Vamos desenvolver a equação acima. Agrupando os termos de mesma ordem de $\Phi_{\mu}\left(z^{\prime}-\gamma\left(z^{\prime}\right)+\right.$ $\left.O\left(\left|z^{\prime}\right|^{4}\right)\right)$, segue que:

$$
\begin{aligned}
\Phi_{\mu}\left(z^{\prime}-\gamma\left(z^{\prime}\right)+O\left(\left|z^{\prime}\right|^{4}\right)\right)= & \lambda\left(z^{\prime}-\gamma\left(z^{\prime}\right)+O\left(\left|z^{\prime}\right|^{4}\right)\right)+\alpha_{3}^{3}\left(z^{\prime}-\gamma\left(z^{\prime}\right)+O\left(\left|z^{\prime}\right|^{4}\right)\right)^{3}+ \\
& \alpha_{2}^{3}\left(z^{\prime}-\gamma\left(z^{\prime}\right)+O\left(\left|z^{\prime}\right|^{4}\right)\right)^{2}\left(\overline{z^{\prime}}-\overline{\gamma\left(z^{\prime}\right)}+O\left(\left|\overline{z^{\prime}}\right|^{4}\right)\right)+ \\
& \alpha_{1}^{3}\left(z^{\prime}-\gamma\left(z^{\prime}\right)+O\left(\left|z^{\prime}\right|^{4}\right)\right)\left(\overline{z^{\prime}}-\overline{\gamma\left(z^{\prime}\right)}+O\left(\left|\overline{z^{\prime}}\right|^{4}\right)\right)^{2}+ \\
& \alpha_{0}^{3}\left(\overline{z^{\prime}}-\overline{\gamma\left(z^{\prime}\right)}+O\left(\left|\overline{z^{\prime}}\right|^{4}\right)\right)^{3}+O\left(\left|z^{\prime}\right|^{4}\right) \\
= & \lambda z^{\prime}-\lambda \gamma\left(z^{\prime}\right)+\alpha_{3}^{3} z^{\prime 3}+\alpha_{2}^{3} z^{2} \overline{z^{\prime}}+\alpha_{1}^{3} z^{\prime}{\overline{z^{\prime}}}^{2}+\alpha_{0}^{3} \overline{z^{\prime}}+O\left(\left|z^{\prime}\right|^{4}\right),
\end{aligned}
$$

e pela definição de $\gamma(z)$ temos:

$$
\begin{aligned}
\gamma\left(\Phi_{\mu}\left(z^{\prime}-\gamma\left(z^{\prime}\right)+O\left(\left|z^{\prime}\right|^{4}\right)\right)\right)= & \gamma\left(\lambda z^{\prime}-\lambda \gamma\left(z^{\prime}\right)+\alpha_{3}^{3} z^{\prime 3}+\alpha_{2}^{3} z^{\prime 2} \overline{z^{\prime}}+\alpha_{1}^{3} z^{\prime} \overline{z^{\prime}}+\alpha_{0}^{3} \overline{z^{\prime}}+O\left(\left|z^{\prime}\right|^{4}\right)\right) \\
= & \gamma_{3}\left(\lambda z^{\prime}-\lambda \gamma\left(z^{\prime}\right)+\alpha_{3}^{3} z^{\prime 3}+\alpha_{2}^{3} z^{\prime 2} \overline{z^{\prime}}+\alpha_{1}^{3} z^{\prime} \bar{z}^{\prime}+\alpha_{0}^{3} \overline{z^{\prime}}+O\left(\left|z^{\prime}\right|^{4}\right)\right)^{3}+ \\
& \gamma_{2}\left(\lambda z^{\prime}-\lambda \gamma\left(z^{\prime}\right)+\alpha_{3}^{3} z^{\prime 3}+\alpha_{2}^{3} z^{\prime 2} \overline{z^{\prime}}+\alpha_{1}^{3} z^{\prime} \bar{z}^{\prime}+\alpha_{0}^{3} \overline{z^{\prime}}+O\left(\left|z^{\prime}\right|^{4}\right)\right)^{2} . \\
& \left(\overline{\lambda z^{\prime}}-\overline{\lambda \gamma\left(z^{\prime}\right)}+\overline{\alpha_{3}^{3}} \overline{z^{\prime}}+\overline{\alpha_{2}^{3}} \overline{z^{\prime}} z^{\prime}+\overline{\alpha_{1}^{3}} \overline{z^{\prime}} z^{\prime 2}+\overline{\alpha_{0}^{3}} z^{\prime 3}+O\left(\left|\overline{z^{\prime}}\right|^{4}\right)\right)+ \\
& \gamma_{1}\left(\lambda z^{\prime}-\lambda \gamma\left(z^{\prime}\right)+\alpha_{3}^{3} z^{\prime 3}+\alpha_{2}^{3} z^{\prime 2} \overline{z^{\prime}}+\alpha_{1}^{3} z^{\prime} \bar{z}^{2}+\alpha_{0}^{3} \overline{z^{\prime}}+O\left(\left|z^{\prime}\right|^{4}\right)\right) . \\
& \left(\overline{\lambda z^{\prime}}-\overline{\lambda \gamma\left(z^{\prime}\right)}+\overline{\alpha_{3}^{3}} \overline{z^{\prime}}+\overline{\alpha_{2}^{3}} \overline{z^{\prime}} z^{\prime}+\overline{\alpha_{1}^{3}} \overline{z^{\prime}} z^{\prime 2}+\overline{\alpha_{0}^{3}} z^{\prime 3}+O\left(\left|\overline{z^{\prime}}\right|^{4}\right)\right)^{2}+ \\
& \gamma_{0}\left(\overline{\lambda z^{\prime}}-\overline{\lambda \gamma\left(z^{\prime}\right)}+\overline{\alpha_{3}^{3}} \overline{z^{\prime}}+\overline{\alpha_{2}^{3}} \overline{z^{\prime}} z^{\prime}+\overline{\alpha_{1}^{3}} \overline{z^{\prime}} z^{\prime 2}+\overline{\alpha_{0}^{3}} z^{\prime 3}+O\left(\left|\overline{z^{\prime}}\right|^{4}\right)\right)^{3}+O\left(\left|z^{\prime}\right|^{4}\right) \\
= & \gamma_{3} \lambda^{3} z^{\prime 3}+\gamma_{2} \lambda|\lambda|^{2} z^{\prime 2} \overline{z^{\prime}}+\gamma_{1} \bar{\lambda}|\lambda|^{2} z^{\prime} \overline{z^{\prime}}+\gamma_{0} \bar{\lambda}^{3} \overline{z^{\prime}}+O\left(\left|z^{\prime}\right|^{4}\right) .
\end{aligned}
$$


Pelas equações (2.13), (2.14) e (2.15) chegamos a:

$$
\begin{aligned}
\widetilde{\Phi}_{\mu}\left(z^{\prime}\right)= & \lambda z^{\prime}-\lambda \gamma\left(z^{\prime}\right)+\alpha_{3}^{3} z^{\prime 3}+\alpha_{2}^{3} z^{2} \overline{z^{\prime}}+\alpha_{1}^{3} z^{\prime}{\overline{z^{\prime}}}^{2}+\alpha_{0}^{3}{\overline{z^{\prime}}}^{3}+ \\
& \gamma_{3} \lambda^{3} z^{\prime 3}+\gamma_{2} \lambda|\lambda|^{2} z^{\prime 2} \overline{z^{\prime}}+\gamma_{1} \bar{\lambda}|\lambda|^{2} z^{\prime}{\overline{z^{\prime}}}^{2}+\gamma_{0} \bar{\lambda}^{3}{\overline{z^{\prime}}}^{3}+O\left(\left|z^{\prime}\right|^{4}\right) \\
= & \lambda z^{\prime}-\lambda \gamma_{3} z^{\prime 3}-\lambda \gamma_{2} z^{\prime 2} \overline{z^{\prime}}-\lambda \gamma_{1} z^{\prime} \bar{z}^{2}-\lambda \gamma_{0}{\overline{z^{\prime}}}^{3}+\alpha_{3}^{3} z^{\prime 3}+\alpha_{2}^{3} z^{\prime 2} \overline{z^{\prime}}+\alpha_{1}^{3} z^{\prime}{\overline{z^{\prime}}}^{2}+ \\
& \alpha_{0}^{3} \overline{z^{\prime}}+\gamma_{3} \lambda^{3} z^{\prime 3}+\gamma_{2} \lambda|\lambda|^{2} z^{\prime 2} \overline{z^{\prime}}+\gamma_{1} \bar{\lambda}|\lambda|^{2} z^{\prime}{\overline{z^{\prime}}}^{2}+\gamma_{0} \bar{\lambda}^{3}{\overline{z^{\prime}}}^{3}+O\left(\left|z^{\prime}\right|^{4}\right) \\
= & \lambda z^{\prime}+\left(-\lambda \gamma_{3}+\lambda^{3} \gamma_{3}+\alpha_{3}^{3}\right) z^{\prime 3}+\left(-\lambda \gamma_{2}+\lambda|\lambda|^{2} \gamma_{2}+\alpha_{2}^{3}\right) z^{\prime 2} \overline{z^{\prime}}+ \\
& \left(-\lambda \gamma_{1}+\bar{\lambda}|\lambda|^{2} \gamma_{1}+\alpha_{1}^{3}\right) z^{\prime}{\overline{z^{\prime}}}^{2}+\left(-\lambda \gamma_{0}+\bar{\lambda}^{3} \gamma_{0}+\alpha_{0}^{3}\right) \bar{z}^{3}+O\left(\left|z^{\prime}\right|^{4}\right) .
\end{aligned}
$$

Observemos que $-\lambda \gamma_{3}+\lambda^{3} \gamma_{3}+\alpha_{3}^{3}$ (coeficiente de $z^{\prime 3}$ ) se anula quando tomamos $\gamma_{3}=\frac{\alpha_{3}^{3}}{\lambda-\lambda^{3}}$. Também temos que $-\lambda \gamma_{1}+\bar{\lambda}|\lambda|^{2} \gamma_{1}+\alpha_{1}^{3}$ (coeficiente de $z^{\prime}{\overline{z^{\prime}}}^{2}$ ) se anula quando tomamos $\gamma_{1}=$ $\frac{\alpha_{1}^{3}}{\lambda-\bar{\lambda}|\lambda|^{2}}$, e $-\lambda \gamma_{0}+\bar{\lambda}^{3} \gamma_{0}+\alpha_{0}^{3}$ (coeficiente de ${\overline{z^{\prime}}}^{3}$ ) se anula quando tomamos $\gamma_{0}=\frac{\alpha_{0}^{3}}{\lambda-\bar{\lambda}^{3}}$. Notemos que os denominadores nas expressões de $\gamma_{3}, \gamma_{1}$ e $\gamma_{0}$ não se anulam, visto que $|\lambda|=1+\mu$, e não há problema para $\mu \neq 0$. A mudança de coordenadas utilizada está bem definida quando $\mu \longrightarrow 0$, pois:

$$
\begin{aligned}
\lambda-\lambda^{3} \neq 0 & \Longleftrightarrow e^{2 i \theta(0)} \neq 1, \\
\lambda-\bar{\lambda} \neq 0 & \Longleftrightarrow e^{2 i \theta(0)} \neq 1, \\
\lambda-\bar{\lambda}^{3} \neq 0 & \Longleftrightarrow e^{4 i \theta(0)} \neq 1 .
\end{aligned}
$$

Portanto, podemos fazer uma mudança de coordenadas $\mu$-dependente, deixando a aplicação com somente um termo cúbico. Assim, substituindo os valores de $\gamma_{0}, \gamma_{1}, \gamma_{2}$ e $\gamma_{3}$ na equação (2.16), obtemos:

$$
\widetilde{\Phi}_{\mu}\left(z^{\prime}\right)=\lambda z^{\prime}+\alpha_{2}^{3} z^{\prime 2} \overline{z^{\prime}}+C_{4}\left(z^{\prime}\right)+O\left(\left|z^{\prime}\right|^{5}\right),
$$

$\operatorname{com} C_{4}\left(z^{\prime}\right)=\sum_{j=0}^{4} \beta_{j}^{4} z^{\prime j}\left(\overline{z^{\prime}}\right)^{4-j}$ e $\beta_{j}^{4} \in \mathbb{C}$

Observemos que, na hipótese desse lema, se tivéssemos trocado $\gamma_{2}=0$ por $\gamma_{2}=\frac{\alpha_{2}^{3}}{\lambda-\lambda|\lambda|^{2}}$, então para $\mu \neq 0$ seria possível cancelar o termo $z^{\prime 2} \overline{z^{\prime}}$ de $\widetilde{\Phi}_{\mu}\left(z^{\prime}\right)$, mas a expressão de $\gamma_{2}$ diverge quando $\mu \longrightarrow 0$, independente do valor de $\theta(0)$. O termo $z^{\prime 2} \overline{z^{\prime}}$ é chamado de termo ressonante. 
Notemos que o coeficiente é o mesmo coeficiente do termo cúbico $z^{\prime 2} \overline{z^{\prime}}$ na equação (2.12).

\section{Lema 2.4 Seja}

$$
\Phi_{\mu}(z)=\lambda z+\alpha_{2}^{3} z^{2} \bar{z}+C_{4}(z)+O\left(|z|^{5}\right)
$$

a aplicação definida em (2.17) e com $\lambda=\lambda(\mu)=(1+\mu) e^{i \theta(\mu)}$. Suponhamos que $e^{i \theta(0)} \neq 1, e^{3 i \theta(0)} \neq 1$ e $e^{5 i \theta(0)} \neq 1$. Então $\Phi_{\mu}$ pode ser transformada pela mudança de coordenadas complexa

$$
\begin{aligned}
\tau: \mathbb{C} & \longrightarrow \mathbb{C} \\
z & \longmapsto z^{\prime}=z+\gamma(z),
\end{aligned}
$$

onde $\lambda=\lambda(\mu), \gamma(z)=\gamma_{4} z^{4}+\gamma_{3} z^{3} \bar{z}+\gamma_{2} z^{2} \bar{z}^{2}+\gamma_{1} z \bar{z}^{3}+\gamma_{0} \bar{z}^{4}, \operatorname{com} \gamma_{4}=\frac{\beta_{4}^{4}}{\lambda-\lambda^{4}}, \gamma_{3}=\frac{\beta_{3}^{4}}{\lambda-\lambda^{2}|\lambda|^{2}}$, $\gamma_{2}=\frac{\beta_{2}^{4}}{\lambda-|\lambda|^{4}}, \gamma_{1}=\frac{\beta_{1}^{4}}{\lambda-\bar{\lambda}^{2}|\lambda|^{2}} e \gamma_{0}=\frac{\beta_{0}^{4}}{\lambda-\bar{\lambda}^{4}}$, na aplicação sem termos quárticos:

$$
\widetilde{\Phi}_{\mu}\left(z^{\prime}\right)=\lambda z^{\prime}+\alpha_{2}^{3} z^{\prime 2} \overline{z^{\prime}}+O\left(\left|z^{\prime}\right|^{5}\right)
$$

Demonstração: Temos que, em uma vizinhança da origem, no novo sistema de coordenadas $z^{\prime}, \overline{z^{\prime}}$, é possível inverter a aplicação $\tau$, obtendo:

$$
z=z^{\prime}-\gamma\left(z^{\prime}\right)+O\left(\left|z^{\prime}\right|^{5}\right)
$$

Denotando $\widetilde{\Phi}_{\mu}$ como sendo $\Phi_{\mu}$ na nova coordenada $z^{\prime}$, teremos :

$$
\begin{aligned}
\widetilde{\Phi}_{\mu}\left(z^{\prime}\right) & =\left(\tau \circ \Phi_{\mu} \circ \tau^{-1}\right)\left(z^{\prime}\right) \\
& =\left(\tau \circ \Phi_{\mu}\right)(z) \\
& =\Phi_{\mu}(z)+\gamma\left(\Phi_{\mu}(z)\right) \\
& =\Phi_{\mu}\left(z^{\prime}-\gamma\left(z^{\prime}\right)+O\left(\left|z^{\prime}\right|^{5}\right)\right)+\gamma\left(\Phi_{\mu}\left(z^{\prime}-\gamma\left(z^{\prime}\right)+O\left(\left|z^{\prime}\right|^{5}\right)\right)\right)
\end{aligned}
$$


Vamos desenvolver a equação (2.18). Pela definição de $\gamma(z)$ temos:

$$
\begin{aligned}
& \gamma\left(\Phi_{\mu}\left(z^{\prime}-\gamma\left(z^{\prime}\right)+O\left(\left|z^{\prime}\right|^{5}\right)\right)\right)=\gamma\left[\lambda z^{\prime}-\lambda \gamma\left(z^{\prime}\right)+\alpha_{2}^{3} z^{\prime 2} \overline{z^{\prime}}+\beta_{4}^{4} z^{4}+\beta_{3}^{4} z^{\prime 3} \overline{z^{\prime}}+\right. \\
& \left.\beta_{2}^{4} z^{\prime 2}{\overline{z^{\prime}}}^{2}+\beta_{1}^{4} z^{\prime}{\overline{z^{\prime}}}^{3}+\beta_{0}^{4} \overline{z^{\prime}}+O\left(\left|z^{\prime}\right|^{5}\right)\right] \\
& =\gamma_{4}\left[\lambda z^{\prime}-\lambda \gamma\left(z^{\prime}\right)+\alpha_{2}^{3} z^{\prime 2} \overline{z^{\prime}}+\beta_{4}^{4} z^{4}+\beta_{3}^{4} z^{\prime 3} \overline{z^{\prime}}+\beta_{2}^{4} z^{\prime 2} \overline{z^{\prime}}+\right. \\
& \left.\beta_{1}^{4} z^{\prime} \overline{z^{\prime}}+\beta_{0}^{4} \overline{z^{4}}+O\left(\left|z^{\prime}\right|^{5}\right)\right]^{4}+\gamma_{3}\left[\lambda z^{\prime}-\lambda \gamma\left(z^{\prime}\right)+\alpha_{2}^{3} z^{\prime 2} \overline{z^{\prime}}+\right. \\
& \left.\beta_{4}^{4} z^{4}+\beta_{3}^{4} z^{\prime 3} \overline{z^{\prime}}+\beta_{2}^{4} z^{\prime 2}{\overline{z^{\prime}}}^{2}+\beta_{1}^{4} z^{\prime} \overline{z^{\prime}}+\beta_{0}^{4} \overline{z^{\prime}}+O\left(\left|z^{\prime}\right|^{5}\right)\right]^{3} \text {. } \\
& {\left[\overline{\lambda z^{\prime}}-\overline{\lambda \gamma\left(z^{\prime}\right)}+\overline{\alpha_{2}^{3}} \overline{z^{\prime}} z^{\prime}+\overline{\beta_{4}^{4}} \overline{z^{\prime}}+\overline{\beta_{3}^{4}} \overline{z^{\prime}} z^{\prime}+\overline{\beta_{2}^{4}}{\overline{z^{\prime}}}^{2} z^{\prime 2}+\right.} \\
& \left.\overline{\beta_{1}^{4}} \overline{z^{\prime}} z^{\prime 3}+\overline{\beta_{0}^{4}} z^{\prime 4}+O\left(\left|\overline{z^{\prime}}\right|^{5}\right)\right]+\gamma_{2}\left[\lambda z^{\prime}-\lambda \gamma\left(z^{\prime}\right)+\alpha_{2}^{3} z^{\prime 2} \overline{z^{\prime}}+\right. \\
& \left.\beta_{4}^{4} z^{4}+\beta_{3}^{4} z^{\prime 3} \overline{z^{\prime}}+\beta_{2}^{4} z^{\prime 2}{\overline{z^{\prime}}}^{2}+\beta_{1}^{4} z^{\prime} \overline{z^{\prime}}+\beta_{0}^{4} \overline{z^{\prime}}+O\left(\left|z^{\prime}\right|^{5}\right)\right]^{2} \text {. } \\
& {\left[\overline{\lambda z^{\prime}}-\overline{\lambda \gamma\left(z^{\prime}\right)}+\overline{\alpha_{2}^{3}} \overline{z^{\prime}} z^{\prime}+\overline{\beta_{4}^{4}} \overline{z^{\prime}}+\overline{\beta_{3}^{4}} \overline{z^{\prime}} z^{\prime}+\overline{\beta_{2}^{4}}{\overline{z^{\prime}}}^{2} z^{\prime 2}+\right.} \\
& \left.\overline{\beta_{1}^{4}} \overline{z^{\prime}} z^{3}+\overline{\beta_{0}^{4}} z^{\prime 4}+O\left(\left|\overline{z^{\prime}}\right|^{5}\right)\right]^{2}+\gamma_{1}\left[\lambda z^{\prime}-\lambda \gamma\left(z^{\prime}\right)+\alpha_{2}^{3} z^{\prime 2} \overline{z^{\prime}}+\right. \\
& \left.\beta_{4}^{4} z^{\prime 4}+\beta_{3}^{4} z^{3} \overline{z^{\prime}}+\beta_{2}^{4} z^{\prime 2} \overline{z^{\prime}}+\beta_{1}^{4} z^{\prime}{\overline{z^{\prime}}}^{3}+\beta_{0}^{4} \overline{z^{\prime}}+O\left(\left|z^{\prime}\right|^{5}\right)\right] . \\
& {\left[\overline{\lambda z^{\prime}}-\overline{\lambda \gamma\left(z^{\prime}\right)}+\overline{\alpha_{2}^{3}}{\overline{z^{\prime}}}^{2} z^{\prime}+\overline{\beta_{4}^{4}}{\overline{z^{\prime}}}^{4}+\overline{\beta_{3}^{4}}{\overline{z^{\prime}}}^{3} z^{\prime}+\overline{\beta_{2}^{4}}{\overline{z^{\prime}}}^{2} z^{\prime 2}+\right.} \\
& \left.\overline{\beta_{1}^{4}} \overline{z^{\prime}} z^{\prime 3}+\overline{\beta_{0}^{4}} z^{4}+O\left(\left|\overline{z^{\prime}}\right|^{5}\right)\right]^{3}+\gamma_{0}\left[\overline{\lambda z^{\prime}}-\overline{\lambda \gamma\left(z^{\prime}\right)}+\overline{\alpha_{2}^{3}} \overline{z^{\prime}} z^{\prime}+\right. \\
& \left.\overline{\beta_{4}^{4}} \overline{z^{\prime}}+\overline{\beta_{3}^{4}}{\overline{z^{\prime}}}^{3} z^{\prime}+\overline{\beta_{2}^{4}} \bar{z}^{2} z^{\prime 2}+\overline{\beta_{1}^{4}} \overline{z^{\prime}} z^{\prime 3}+\overline{\beta_{0}^{4}} z^{4}+O\left(\left|\overline{z^{\prime}}\right|^{5}\right)\right]^{4} \\
& =\gamma_{4} \lambda^{4} z^{4}+\gamma_{3} \lambda^{2}|\lambda|^{2} z^{\prime 3} \overline{z^{\prime}}+\gamma_{2}|\lambda|^{4} z^{\prime 2}{\overline{z^{\prime}}}^{2}+\gamma_{1} \bar{\lambda}^{2}|\lambda|^{2} z^{\prime}{\overline{z^{\prime}}}^{3}+ \\
& \gamma_{0} \bar{\lambda}^{4} \overline{z^{\prime}}+O\left(\left|z^{\prime}\right|^{5}\right) \text {. }
\end{aligned}
$$


E agrupando os termos de mesma ordem de $\Phi_{\mu}\left(z^{\prime}-\gamma\left(z^{\prime}\right)+O\left(\left|z^{\prime}\right|^{5}\right)\right)$, segue que:

$$
\begin{aligned}
\Phi_{\mu}\left(z^{\prime}-\gamma\left(z^{\prime}\right)+O\left(\left|z^{\prime}\right|^{5}\right)\right)= & \lambda\left(z^{\prime}-\gamma\left(z^{\prime}\right)+O\left(\left|z^{\prime}\right|^{5}\right)\right)+ \\
& \alpha_{2}^{3}\left(z^{\prime}-\gamma\left(z^{\prime}\right)+O\left(\left|z^{\prime}\right|^{5}\right)\right)^{2}\left(\overline{z^{\prime}}-\overline{\gamma\left(z^{\prime}\right)}+O\left(\left|\overline{z^{\prime}}\right|^{5}\right)\right)+ \\
& \beta_{4}^{4}\left(z^{\prime}-\gamma\left(z^{\prime}\right)+O\left(\left|z^{\prime}\right|^{5}\right)\right)^{4}+ \\
& \left.\beta_{3}^{4}\left(z^{\prime}-\gamma\left(z^{\prime}\right)+O\left(\left|z^{\prime}\right|^{5}\right)\right)^{3} \overline{\left(z^{\prime}\right.}-\overline{\gamma\left(z^{\prime}\right)}+O\left(\left|\overline{z^{\prime}}\right|^{5}\right)\right)+ \\
& \left.\beta_{2}^{4}\left(z^{\prime}-\gamma\left(z^{\prime}\right)+O\left(\left|z^{\prime}\right|^{5}\right)\right)^{2} \overline{\left(z^{\prime}\right.}-\overline{\gamma\left(z^{\prime}\right)}+O\left(\left|\overline{z^{\prime}}\right|^{5}\right)\right)^{2}+ \\
& \beta_{1}^{4}\left(z^{\prime}-\gamma\left(z^{\prime}\right)+O\left(\left|z^{\prime}\right|^{5}\right)\right)\left(\overline{z^{\prime}}-\overline{\gamma\left(z^{\prime}\right)}+O\left(\left|\overline{z^{\prime}}\right|^{5}\right)\right)^{3}+ \\
& \left.\beta_{0}^{4} \overline{z^{\prime}}-\overline{\gamma\left(z^{\prime}\right)}+O\left(\left|\overline{z^{\prime}}\right|^{5}\right)\right)^{4}+O\left(\left|z^{\prime}\right|^{5}\right) \\
= & \lambda z^{\prime}-\lambda \gamma\left(z^{\prime}\right)+\alpha_{2}^{3} z^{\prime 2} \overline{z^{\prime}}+\beta_{4}^{4} z^{\prime 4}+\beta_{3}^{4} z^{\prime 3} \overline{z^{\prime}}+\beta_{2}^{4} z^{\prime 2} \overline{z^{\prime}}+ \\
& \beta_{1}^{4} z^{\prime}{\overline{z^{\prime}}}^{3}+\beta_{0}^{4} \overline{z^{\prime}}+O\left(\left|z^{\prime}\right|^{5}\right)
\end{aligned}
$$

Pelas equações (2.18) , (2.20) e (2.19) chegamos a:

$$
\begin{aligned}
& \widetilde{\Phi}_{\mu}\left(z^{\prime}\right)=\lambda z^{\prime}-\lambda \gamma\left(z^{\prime}\right)+\alpha_{2}^{3} z^{\prime 2} \overline{z^{\prime}}+\beta_{4}^{4} z^{4}+\beta_{3}^{4} z^{\prime 3} \overline{z^{\prime}}+\beta_{2}^{4} z^{\prime 2} \overline{z^{\prime}}+\beta_{1}^{4} z^{\prime} \overline{z^{\prime}}+\beta_{0}^{4} \overline{z^{\prime}}+ \\
& \gamma_{4} \lambda^{4} z^{4}+\gamma_{3} \lambda^{2}|\lambda|^{2} z^{\prime 3} \overline{z^{\prime}}+\gamma_{2}|\lambda|^{4} z^{\prime 2}{\overline{z^{\prime}}}^{2}+\gamma_{1} \bar{\lambda}^{2}|\lambda|^{2} z^{\prime}{\overline{z^{\prime}}}^{3}+\gamma_{0} \bar{\lambda} \overline{z^{\prime}}+O\left(\left|z^{\prime}\right|\right)^{5} \\
& =\lambda z^{\prime}+\alpha_{2}^{3} z^{\prime 2} \overline{z^{\prime}}-\gamma_{4} \lambda z^{4}-\gamma_{3} \lambda z^{\prime 3} \overline{z^{\prime}}-\gamma_{2} \lambda z^{\prime 2}{\overline{z^{\prime}}}^{2}-\gamma_{1} \lambda z^{\prime}{\overline{z^{\prime}}}^{3}-\gamma_{0} \lambda \overline{z^{\prime}}+ \\
& \beta_{4}^{4} z^{4}+\beta_{3}^{4} z^{3} \overline{z^{\prime}}+\beta_{2}^{4} z^{\prime 2} \overline{z^{\prime}}+\beta_{1}^{4} z^{z^{\prime}} \bar{z}^{3}+\beta_{0}^{4} \overline{z^{\prime}}+ \\
& \gamma_{4} \lambda^{4} z^{\prime 4}+\gamma_{3} \lambda^{2}|\lambda|^{2} z^{\prime 3} \overline{z^{\prime}}+\gamma_{2}|\lambda|^{4} z^{\prime 2}{\overline{z^{\prime}}}^{2}+\gamma_{1} \bar{\lambda}^{2}|\lambda|^{2} z^{\prime}{\overline{z^{\prime}}}^{3}+\gamma_{0} \bar{\lambda}^{4} \overline{z^{\prime}}+O\left(\left|z^{\prime}\right|\right)^{5} \\
& =\lambda z^{\prime}+\alpha_{2}^{3} z^{\prime 2} \overline{z^{\prime}}+\left(-\gamma_{4} \lambda+\beta_{4}^{4}+\gamma_{4} \lambda^{4}\right) z^{4}+\left(-\gamma_{3} \lambda+\beta_{3}^{4}+\gamma_{3} \lambda^{2}|\lambda|^{2}\right) z^{\prime 3} \overline{z^{\prime}}+ \\
& \left(-\gamma_{2} \lambda+\beta_{2}^{4}+\gamma_{2}|\lambda|^{4}\right) z^{\prime 2}{\overline{z^{\prime}}}^{2}+\left(-\gamma_{1} \lambda+\beta_{1}^{4}+\gamma_{1} \bar{\lambda}^{2}|\lambda|^{2}\right) z^{\prime}{\overline{z^{\prime}}}^{3}+\left(-\gamma_{0} \lambda+\beta_{0}^{4}+\gamma_{0} \bar{\lambda}^{4}\right) \overline{z^{\prime}}+O\left(\left|z^{\prime}\right|\right)^{5} \text {. }
\end{aligned}
$$

Observemos que $-\gamma_{4} \lambda+\beta_{4}^{4}+\gamma_{4} \lambda^{4}$ (coeficiente de $z^{\prime 4}$ ) se anula quando tomamos $\gamma_{4}=\frac{\beta_{4}^{4}}{\lambda-\lambda^{4}}$. E $-\gamma_{3} \lambda+\beta_{3}^{4}+\gamma_{3} \lambda^{2}|\lambda|^{2}$ (coeficiente de $z^{\prime 3} \overline{z^{\prime}}$ ) se anula quando tomamos $\gamma_{3}=\frac{\beta_{3}^{4}}{\lambda-\lambda^{2}|\lambda|^{2}}$. A expressão $-\gamma_{2} \lambda+\beta_{2}^{4}+\gamma_{2}|\lambda|^{4}$ (coeficiente de $z^{\prime 2}{\overline{z^{\prime}}}^{2}$ ) se anula quando tomamos $\gamma_{2}=\frac{\beta_{2}^{4}}{\lambda-|\lambda|^{4}}$. De fato, $-\gamma_{1} \lambda+$ $\beta_{1}^{4}+\gamma_{1} \bar{\lambda}^{2}|\lambda|^{2}$ (coeficiente de $z^{\prime}{\overline{z^{\prime}}}^{3}$ ) se anula quando tomamos $\gamma_{1}=\frac{\beta_{1}^{4}}{\lambda-\bar{\lambda}^{2}|\lambda|^{2}}$. E $-\gamma_{0} \lambda+\beta_{0}^{4}+\gamma_{0} \bar{\lambda}^{4}$ 
(coeficiente de $\overline{z^{\prime}}$ ) se anula quando tomamos $\gamma_{0}=\frac{\beta_{0}^{4}}{\lambda-\bar{\lambda}^{4}}$. Notemos que os denominadores nas expressões de $\gamma_{4}, \gamma_{3}, \gamma_{2}, \gamma_{1}$ e $\gamma_{0}$ não se anulam, visto que $|\lambda|=1+\mu$, e não há problema para $\mu \neq 0$. A mudança de coordenadas utilizada está bem definida quando $\mu \longrightarrow 0$, pois:

$$
\begin{aligned}
\lambda-\lambda^{4} \neq 0 & \Longleftrightarrow e^{3 i \theta(0)} \neq 1, \\
\lambda-\lambda^{2} \neq 0 & \Longleftrightarrow e^{i \theta(0)} \neq 1, \\
\lambda-1 \neq 0 & \Longleftrightarrow e^{i \theta(0)} \neq 1, \\
\lambda-\bar{\lambda}^{2} \neq 0 & \Longleftrightarrow e^{3 i \theta(0)} \neq 1, \\
\lambda-\bar{\lambda}^{4} \neq 0 & \Longleftrightarrow e^{5 i \theta(0)} \neq 1 .
\end{aligned}
$$

Portanto podemos fazer uma mudança de coordenadas $\mu$-dependente, deixando a aplicação $\Phi_{\mu}$ sem termos quárticos. Assim, substituindo os valores de $\gamma_{0}, \gamma_{1}, \gamma_{2}, \gamma_{3}$ e $\gamma_{4}$ na equação (2.21), obtemos:

$$
\widetilde{\Phi}_{\mu}\left(z^{\prime}\right)=\lambda z^{\prime}+\alpha_{2}^{3} z^{\prime 2} \overline{z^{\prime}}+O\left(\left|z^{\prime}\right|^{5}\right)
$$

Pelos Lemas 2.2, 2.3 e 2.4 temos que, supondo $e^{k i \theta(0)} \neq 1$, para $k=1,2,3$, 4 e 5, é possível fazer mudanças de coordenadas para a aplicação $\Phi_{\mu}(z)=\lambda(\mu) z+\sum_{j=0}^{k} \alpha_{j}^{k} z^{j}(\bar{z})^{k-j}+O\left(|z|^{k+1}\right)$, de tal forma a deixá-la na forma $\Phi_{\mu}(z)=\lambda(\mu) z+\alpha_{2}^{3} z^{2} \bar{z}+O\left(|z|^{5}\right)$. Assim podemos enunciar e demonstrar o seguinte teorema:

Teorema 2.1 Seja

$$
\begin{aligned}
\Phi_{\mu}: \mathbb{R}^{2} & \longrightarrow \mathbb{R}^{2} \\
\left(x_{1}, x_{2}\right) & \longmapsto(1+\mu)\left(\begin{array}{rr}
\cos \theta(\mu) & -\operatorname{sen} \theta(\mu) \\
\operatorname{sen} \theta(\mu) & \cos \theta(\mu)
\end{array}\right)\left(\begin{array}{l}
x_{1} \\
x_{2}
\end{array}\right)+g_{\mu}\left(x_{1}, x_{2}\right),
\end{aligned}
$$

uma família de aplicações do plano de classe $C^{4}$. Suponhamos que $\Phi_{\mu}(0,0)=(0,0)$ para todo $\mu, D \Phi_{\mu}(0,0)$ tem autovalores $(1+\mu) e^{i \theta(\mu)} e(1+\mu) e^{-i \theta(\mu)}$ (após reparametrização de $\mu$ ), e e $e^{k i \theta(0)} \neq 1$ para $k=1,2,3,4$ e 5. Então existe um mudança suave de coordenadas $\mu$-dependente numa vizinhança da origem, tal que nas novas coordenadas $\Phi_{\mu}$ toma a forma:

$$
\Phi_{\mu}(z)=N \Phi_{\mu}(z)+O\left(|z|^{5}\right),
$$


onde, em coordenadas polares,

$$
N \Phi_{\mu}:(r, \varphi) \longmapsto\left((1+\mu) r-f_{1}(\mu) r^{3}, \varphi+\theta(\mu)+f_{3}(\mu) r^{2}\right),
$$

$\operatorname{com} f_{1}(\mu)=-\operatorname{Re}\left(\frac{\alpha_{2}^{3}}{e^{i \theta(\mu)}}\right), f_{3}(\mu)=\frac{1}{1+\mu} \operatorname{Im}\left(\frac{\alpha_{2}^{3}}{e^{i \theta(\mu)}}\right)$ e $\alpha_{2}^{3}$ dado como no Lema 2.3.

Demonstração: Pelos Lemas 2.2, 2.3 e 2.4 vimos que através de mudanças de coordenadas $\mu$ dependente é possível escrever a aplicação $\Phi_{\mu}$ na forma:

$$
\Phi_{\mu}(z)=\lambda z+\alpha_{2}^{3} z^{2} \bar{z}+O\left(|z|^{5}\right)=\left(\lambda+\alpha_{2}^{3}|z|^{2}\right) z+O\left(|z|^{5}\right),
$$

onde $\lambda=\lambda(\mu)=(1+\mu) e^{i \theta(\mu)}$. Vamos reescrever $\left(\lambda+\alpha_{2}^{3}|z|^{2}\right) z+O\left(|z|^{5}\right)$ em coordenadas polares. Primeiramente, escrevemos de outra forma a expressão $\lambda+\alpha_{2}^{3}|z|^{2}$ :

$$
\lambda+\alpha_{2}^{3}|z|^{2}=(1+\mu) e^{i \theta(\mu)}+\alpha_{2}^{3}|z|^{2}=(1+\mu) e^{i \theta(\mu)}\left[1+\frac{\alpha_{2}^{3}}{e^{i \theta(\mu)}} \frac{|z|^{2}}{1+\mu}\right] .
$$

Temos que $\frac{\alpha_{2}^{3}}{e^{i \theta(\mu)}} \in \mathbb{C}$ e $\frac{|z|^{2}}{1+\mu} \in \mathbb{R}$. Definimos então duas funções reais $f_{1}$ e $f_{3}$, da seguinte forma:

$$
\begin{aligned}
f_{1}: \mathbb{R} & \longrightarrow \mathbb{R} \\
\mu & \longmapsto-\operatorname{Re}\left(\frac{\alpha_{2}^{3}}{e^{i \theta(\mu)}}\right),
\end{aligned}
$$

e

$$
\begin{aligned}
f_{3}: \mathbb{R} & \longrightarrow \mathbb{R} \\
\mu & \longmapsto \frac{1}{1+\mu} \operatorname{Im}\left(\frac{\alpha_{2}^{3}}{e^{i \theta(\mu)}}\right) .
\end{aligned}
$$


Utilizando $f_{1}$ e $f_{3}$, escrevemos:

$$
\begin{aligned}
& \lambda+\alpha_{2}^{3}|z|^{2}=(1+\mu) e^{i \theta(\mu)}\left[1-\frac{f_{1}(\mu)}{1+\mu}|z|^{2}+i f_{3}(\mu)|z|^{2}\right] \\
& =(1+\mu) e^{i \theta(\mu)}\left[1-\frac{f_{1}(\mu)}{1+\mu}|z|^{2}\right]\left[1+i f_{3}(\mu)|z|^{2}\right]+O\left(|z|^{4}\right) \\
& =(1+\mu) e^{i \theta(\mu)}\left[1-\frac{f_{1}(\mu)}{1+\mu}|z|^{2}\right] e^{i f_{3}(\mu)|z|^{2}}+O\left(|z|^{4}\right) \\
& =\left(1+\mu-f_{1}(\mu)|z|^{2}\right) e^{i\left[\theta(\mu)+f_{3}(\mu)|z|^{2}\right]}+O\left(|z|^{4}\right) .
\end{aligned}
$$

Portanto podemos escrever $\Phi_{\mu}$ na forma:

$$
\Phi_{\mu}(z)=\left(1+\mu-f_{1}(\mu)|z|^{2}\right) e^{i\left[\theta(\mu)+f_{3}(\mu)|z|^{2}\right]} z+O\left(|z|^{5}\right) .
$$

Vamos escrever a aplicação em coordenadas polares, tomando $z=r(\cos \varphi+i \operatorname{sen} \varphi)$. Teremos:

$$
\begin{aligned}
\Phi_{\mu}(r, \varphi) & =\left(1+\mu-f_{1}(\mu) r^{2}\right) e^{i\left[\theta(\mu)+f_{3}(\mu) r^{2}\right]} r(\cos \varphi+i \operatorname{sen} \varphi)+O\left(r^{5}\right) \\
& =\left((1+\mu) r-f_{1}(\mu) r^{3}\right) e^{i\left[\varphi+\theta(\mu)+f_{3}(\mu) r^{2}\right]}+O\left(r^{5}\right) .
\end{aligned}
$$

$\mathrm{E}\left|\Phi_{\mu}(r, \varphi)\right|=\left|(1+\mu) r-f_{1}(\mu) r^{3}\right|$. Como estamos analisando o comportamento quando $r \longrightarrow 0$, então $(1+\mu) r-f_{1}(\mu) r^{3}>0$, e temos também que $\arg \Phi_{\mu}(r, \varphi)=\varphi+\theta(\mu)+f_{3}(\mu) r^{2}$. Portanto a forma canônica aproximada para a aplicação $\Phi_{\mu}$ será:

$$
\Phi_{\mu}(z)=N \Phi_{\mu}(z)+O\left(|z|^{5}\right)
$$

onde em coordenadas polares $N \Phi_{\mu}$ toma a forma:

$$
(r, \varphi) \longmapsto\left((1+\mu) r-f_{1}(\mu) r^{3}, \varphi+\theta(\mu)+f_{3}(\mu) r^{2}\right)
$$

Notemos duas características especiais de $N \Phi_{\mu}$ :

i) A primeira coordenada de $N \Phi_{\mu}$ depende apenas do valor anterior de $r$, e não de $\varphi$.

ii) A segunda coordenada de $N \Phi_{\mu}$ é obtida somando a $\varphi$ um valor que depende apenas de $r$ e $\mu$. 
Supondo $f_{1}(0)>0$, segue que, para um $\mu$ positivo e pequeno, $N \Phi_{\mu}$ tem uma circunferência invariante de raio $r_{0}=\sqrt{\frac{\mu}{f_{1}(\mu)}}$. Já que $\Phi_{\mu}$ difere apenas um pouco de $N \Phi_{\mu}$, verificaremos que $\Phi_{\mu}$ tem uma curva fechada invariante.

\subsection{Bifurcação de Poincaré-Andronov-Hopf.}

Nesta seção demonstraremos o Teorema de Poincaré-Andronov-Hopf para difeomorfismo do plano. Mostraremos a existência de uma curva fechada invariante para a aplicação $\Phi_{\mu}$ definida em (2.22), para $\mu$ suficientemente pequeno e positivo, utilizando o princípio da contração. Uma das condições para que $\Phi_{\mu}$ tenha uma curva fechada invariante é que o coeficiente $f_{1}(0)$ seja diferente de zero. Logo após a demonstração do teorema, iremos apresentar uma proposição que nos dará um método rápido para calcularmos o coeficiente $f_{1}(0)$.

Teorema 2.2 (Bifurcação de Poincaré-Andronov-Hopf para difeomorfismos) Seja

$$
\begin{aligned}
\Phi: \mathbb{R} \times \mathbb{R}^{2} & \longrightarrow \mathbb{R}^{2} \\
(\mu, x) & \longmapsto \Phi(\mu, x),
\end{aligned}
$$

uma aplicação de classe $C^{4}$ dependendo de uma parâmetro real $\mu$, satisfazendo as seguintes condições:

i) $\Phi(\mu, 0)=0$, para $\mu$ perto de 0 ;

ii) $D \Phi(\mu, 0)$ (isto é, $\left.\left.D_{x} \Phi(\mu, x)\right|_{x=0}\right)$ tem dois autovalores complexos conjugados $z(\mu)$ e $\bar{z}(\mu)$, para $\mu$ perto de 0 , e com $|z(0)|=1$;

iii) $\frac{d}{d \mu}|z(\mu)|>0$ em $\mu=0$;

iv) $z^{k}(0) \neq 1$ para $k=1,2,3,4$ e 5 .

Então existe uma mudança de coordenadas de classe $C^{4}$ dependente de $\mu$, levando $\Phi$ à forma:

$$
\Phi(\mu, x)=N \Phi(\mu, x)+O\left(\|x\|^{5}\right)
$$

e existem funções $f_{1}(\mu), f_{3}(\mu)$, e $\theta(\mu)$ de classe $C^{4}$ tais que, em coordenadas polares, a aplicação $N \Phi(\mu, x)$ é dada por:

$$
\left(\begin{array}{c}
r \\
\varphi
\end{array}\right) \longmapsto\left(\begin{array}{c}
|z(\mu)| r-f_{1}(\mu) r^{3} \\
\varphi+\theta(\mu)+f_{3}(\mu) r^{2}
\end{array}\right)
$$


Se $f_{1}(0)>0$, então existe uma vizinhança $\mathcal{V}$ da origem e $\delta>0$, tal que para $|\mu|<\delta$ e $x_{0} \in \mathcal{V}$, o conjunto $w$-limite de $x_{0}$ é a origem se $\mu<0$, ou pertence a uma curva invariante fechada $\Gamma(\mu)$ de classe $C^{1}$ com a origem em seu interior se $\mu>0$, e além disso $\Gamma(0)=0$. Se $f_{1}(0)<0$, então existe uma vizinhança $\nu$ da origem e $\delta>0$, tal que para $|\mu|<\delta$ e $x_{0} \in \mathcal{V}$, o conjunto $\alpha$-limite de $x_{0}$ é a origem se $\mu>0$, ou pertence a uma curva invariante fechada $\Gamma(\mu)$ de classe $C^{1}$ com a origem em seu interior se $\mu<0$, além disso $\Gamma(0)=0$.

Demonstração: Vamos separar a demonstração do teorema em quatro casos, que dependem do sinal de $f_{1}(0)$ e $\mu$.

Caso 1: $f_{1}(0)>0$ e $\mu>0$.

Do Teorema 2.1 e das hipóteses acima, segue que existe uma mudança de coordenadas de classe $C^{4}$ dependente de $\mu$, levando $\Phi_{\mu}$ à forma: $\Phi(\mu, x)=N \Phi(\mu, x)+O\left(\|x\|^{5}\right)$ e existem funções $f_{1}(\mu), f_{3}(\mu)$, e $\theta(\mu)$ de classe $C^{4}$ tais que, em coordenadas polares, a aplicação $N \Phi(\mu, x)$ é dada por:

$$
\left(\begin{array}{c}
r \\
\varphi
\end{array}\right) \longmapsto\left(\begin{array}{c}
|z(\mu)| r-f_{1}(\mu) r^{3} \\
\varphi+\theta(\mu)+f_{3}(\mu) r^{2}
\end{array}\right) \text {. }
$$

Portanto, para terminarmos a demonstração desse caso resta provar que para $f_{1}(0)>0$ e $\mu>0$, existe uma curva invariante fechada de classe $C^{1}$ para $\Phi_{\mu}$, com a origem em seu interior, na vizinhança da circunferência de raio $r_{0}=\sqrt{\frac{\mu}{f_{1}(\mu)}}$, que é invariante para $N \Phi_{\mu}$. Portanto, é conveniente escolher coordenadas que exibam com a clareza possível, o comportamento de $\Phi_{\mu}$ perto dessa circunferência. Para facilitar a leitura da demonstração deste caso, vamos dividi-lo em passos.

Passo 1: Vamos primeiramente escrever $\Phi_{\mu}(z)$ em coordenadas polares.

Seja

$$
\Phi_{\mu}(z)=N \Phi_{\mu}(z)+O\left(|z|^{5}\right)
$$

a forma canônica de $\Phi_{\mu}$, obtida na seção anterior. Tomemos $z=r(\cos \varphi+i \operatorname{sen} \varphi)$, onde $|z|^{5}=r^{5}$, com $r>0$ e $0 \leq \varphi \leq 2 \pi$. Então a primeira coordenada de $\Phi_{\mu}(r, \varphi)$, em coordenadas polares, difere de $N \Phi_{\mu}(r, \varphi)$ por termos da ordem $O\left(r^{5}\right)$. Agora, vamos olhar como se comporta a segunda coordenada de $\Phi_{\mu}(r, \varphi)$, quando somamos termos de ordem $O\left(|z|^{5}\right)$. Seja $z_{1}=N \Phi_{\mu}(z)=r_{1} e^{i \theta_{1}}$ e 
$z_{2}=O\left(|z|^{5}\right)=r_{2} e^{i \theta_{2}}$, então $\Phi_{\mu}(z)=z_{1}+z_{2}$, queremos determinar o $\arg \left(z_{1}+z_{2}\right)$. Observermos que:

$$
\begin{aligned}
\arg \left(z_{1}+z_{2}\right) & =\arg \left(z_{1} e^{-i \theta_{1}}+z_{2} e^{-i \theta_{1}}\right)+\theta_{1} \\
& =\arg \left(r_{1}+r_{2} e^{i\left(\theta_{2}-\theta_{1}\right)}\right)+\theta_{1} \\
& =\arg \left(\left(r_{1}, 0\right)+\left(r_{2} \cos \left(\theta_{2}-\theta_{1}\right), r_{2} \operatorname{sen}\left(\theta_{2}-\theta_{1}\right)\right)\right)+\theta_{1} .
\end{aligned}
$$

Podemos supor que $\theta_{1}<\theta_{2}$ e tomar $\widetilde{\theta}=\theta_{2}-\theta_{1}$, e então podemos escrever $\widetilde{z}=\left(r_{1}, 0\right)+\left(r_{2} \cos \left(\theta_{2}-\right.\right.$ $\left.\left.\theta_{1}\right), r_{2} \operatorname{sen}\left(\theta_{2}-\theta_{1}\right)\right)$. Logo:

$$
\begin{aligned}
|\widetilde{z}| & =\sqrt{\left(r_{1}+r_{2} \cos \widetilde{\theta}\right)^{2}+\left(r_{2} \operatorname{sen} \widetilde{\theta}\right)^{2}} \\
& =\sqrt{r_{1}^{2}+2 r_{1} r_{2} \cos \widetilde{\theta}+r_{2}^{2}}
\end{aligned}
$$

e $\operatorname{Im} \widetilde{z}=r_{2} \operatorname{sen} \widetilde{\theta}$. Portanto:

$$
\begin{aligned}
\arg (\widetilde{z}) & =\operatorname{arcsen}\left(\frac{r_{2} \operatorname{sen} \widetilde{\theta}}{|\widetilde{z}|}\right) \\
& =\frac{r_{2} \operatorname{sen} \widetilde{\theta}}{|\widetilde{z}|}+O\left(\left(\frac{r_{2} \operatorname{sen} \widetilde{\theta}}{|\widetilde{z}|}\right)^{3}\right)
\end{aligned}
$$

Como $r_{2}$ é da ordem $O\left(r^{5}\right)$ e $|\widetilde{z}| \geq K r$, com $K$ constante positiva, então $\arg (\widetilde{z})$ é da ordem $O\left(r^{4}\right)$, pois:

$$
\begin{aligned}
|\arg (\widetilde{z})| & \leq \frac{r_{2}}{K r}+K_{1} \frac{r_{2}^{3}}{(K r)^{3}}+O\left(\left(\frac{r_{2} \operatorname{sen} \widetilde{\theta}}{|\widetilde{z}|}\right)^{5}\right) \\
& \leq \frac{r^{5}}{K r}+K_{1} \frac{\left(r^{5}\right)^{3}}{(K r)^{3}}+O\left(r^{20}\right) \\
& \leq r^{4}+O\left(r^{12}\right) .
\end{aligned}
$$

Logo

$$
\begin{aligned}
\arg \left(z_{1}+z_{2}\right) & =\theta_{1}+\arg (\widetilde{z}) \\
\arg \left(\Phi_{\mu}\right) & =\arg \left(N \Phi_{\mu}\right)+O\left(r^{4}\right)=\varphi+\theta(\mu)+f_{3}(\mu) r^{2}+O\left(r^{4}\right) .
\end{aligned}
$$


Portanto em coordenadas polares, $\Phi_{\mu}$ toma a forma:

$$
\Phi_{\mu}:(r, \varphi) \mapsto\left((1+\mu) r-f_{1}(\mu) r^{3}+O\left(r^{5}\right), \varphi+\theta(\mu)+f_{3}(\mu) r^{2}+O\left(r^{4}\right)\right)
$$

e essas aproximações são uniformes para $0 \leq \varphi \leq 2 \pi$ e $\mu$ em uma vizinhança da origem. A forma normal

$$
N \Phi_{\mu}(r, \varphi)=\left((1+\mu) r-f_{1}(\mu) r^{3}, \varphi+\theta(\mu)+f_{3}(\mu) r^{2}\right),
$$

é uma aproximação de $\Phi_{\mu}$, com um erro de ordem 5 na primeira coordenada, e de ordem 4 na segunda coordenada.

Passo 2: Neste passo faremos mudanças de coordenadas de tal forma que a circunferência invariante de $N \Phi_{\mu}$ esteja centrado na origem e tenha raio 1.

Primeiramente faremos uma mudança de coordenadas de tal forma que a circunferência invariante de $N \Phi_{\mu}$ tenha raio 1 . Para isto tomamos a mudança de coordenadas:

$$
\begin{aligned}
\tau_{1}: \mathbb{R}^{2} & \longrightarrow \mathbb{R}^{2} \\
(x, \varphi) & \longmapsto\left(\sqrt{\frac{\mu}{f_{1}(\mu)}} x, \varphi\right)=(r, \varphi),
\end{aligned}
$$

com inversa

$$
\begin{aligned}
\tau_{1}^{-1}: \mathbb{R}^{2} & \longrightarrow \mathbb{R}^{2} \\
(x, \varphi) & \longmapsto\left(\sqrt{\left.\frac{f_{1}(\mu)}{\mu} x, \varphi\right) .}\right.
\end{aligned}
$$

Fazendo então essa mudança de coordenadas, obtemos:

$$
\begin{aligned}
\left(\tau_{1}^{-1} \circ \Phi_{\mu} \circ \tau_{1}\right)(x, \varphi)= & \tau_{1}^{-1} \circ \Phi_{\mu}\left(\sqrt{\frac{\mu}{f_{1}(\mu)}} x, \varphi\right) \\
= & \tau_{1}^{-1}\left((1+\mu) \sqrt{\frac{\mu}{f_{1}(\mu)}} x-f_{1}(\mu) \frac{\mu^{\frac{3}{2}}}{f_{1}(\mu)^{\frac{3}{2}}} x^{3}+\mu^{\frac{5}{2}} O\left(x^{5}\right),\right. \\
& \left.\varphi+\theta(\mu)+f_{3}(\mu) \frac{\mu}{f_{1}(\mu)} x^{2}+\mu^{2} O\left(x^{4}\right)\right) \\
= & \left((1+\mu) x-\mu x^{3}+\mu^{2} O\left(x^{5}\right), \varphi+\theta(\mu)+f_{3}(\mu) \frac{\mu}{f_{1}(\mu)} x^{2}+\mu^{2} O\left(x^{4}\right)\right) .
\end{aligned}
$$


Agora transladamos a circunferência para a origem, tomando uma nova mudança de coordenadas:

$$
\begin{aligned}
\tau_{2}: \mathbb{R}^{2} & \longrightarrow \mathbb{R}^{2} \\
(y, \varphi) & \longmapsto(1+y, \varphi),
\end{aligned}
$$

com inversa

$$
\begin{aligned}
\tau_{2}^{-1}: \mathbb{R}^{2} & \longrightarrow \mathbb{R}^{2} \\
(y, \varphi) & \longmapsto(-1+y, \varphi) .
\end{aligned}
$$

Fazendo então essa mudança de coordenadas, $y=0$ será invariante, e obtemos:

$$
\begin{aligned}
\left(\tau_{2}^{-1} \circ \Phi_{\mu} \circ \tau_{2}\right)(y, \varphi)= & \tau_{2}^{-1} \circ \Phi_{\mu}(1+y, \varphi) \\
= & \tau_{2}^{-1}\left((1+\mu)(1+y)-\mu(1+y)^{3}+\mu^{2} O\left((1+y)^{5}\right),\right. \\
& \left.\varphi+\theta(\mu)+f_{3}(\mu) \frac{\mu}{f_{1}(\mu)}(1+y)^{2}+\mu^{2} O\left((1+y)^{4}\right)\right) \\
= & \left((1-2 \mu) y-\mu\left(3 y^{2}+y^{3}\right)+\mu^{2} O(1), \varphi+\theta(\mu)+f_{3}(\mu) \frac{\mu}{f_{1}(\mu)}(1+y)^{2}+\mu^{2} O(1)\right) .
\end{aligned}
$$

Finalmente faremos uma última mudança de coordenadas para reescalar y tomando:

$$
\begin{aligned}
\tau_{3}: \mathbb{R}^{2} & \longrightarrow \mathbb{R}^{2} \\
(z, \varphi) & \longmapsto(z \sqrt{\mu}, \varphi),
\end{aligned}
$$

com inversa

$$
\begin{aligned}
\tau_{3}^{-1}: \mathbb{R}^{2} & \longrightarrow \mathbb{R}^{2} \\
(z, \varphi) & \longmapsto\left(\frac{z}{\sqrt{\mu}}, \varphi\right) .
\end{aligned}
$$

Fazendo então essa mudança de coordenadas, $z=0$ será invariante, e obteremos:

$$
\begin{aligned}
\left(\tau_{3}^{-1} \circ \Phi_{\mu} \circ \tau_{3}\right)(z, \varphi)= & \tau_{3}^{-1} \circ \Phi_{\mu}(z \sqrt{\mu}, \varphi) \\
= & \tau_{3}^{-1}\left((1-2 \mu) \sqrt{\mu} z-\mu\left(3 \mu z^{2}+\mu \sqrt{\mu} z^{3}\right)+\mu^{2} O(1),\right. \\
& \left.\varphi+\theta(\mu)+f_{3}(\mu) \frac{\mu}{f_{1}(\mu)}(1+\sqrt{\mu} z)^{2}+\mu^{2} O(1)\right) \\
= & \left((1-2 \mu) z-\mu^{\frac{3}{2}}\left(3 z^{2}+\mu^{\frac{1}{2}} z^{3}\right)+\mu^{\frac{3}{2}} O(1), \varphi+\theta(\mu)+f_{3}(\mu) \frac{\mu}{f_{1}(\mu)}(1+\sqrt{\mu} z)^{2}+\mu^{2} O(1)\right) .
\end{aligned}
$$


Se definirmos

$$
\begin{gathered}
H_{\mu}(z, \varphi)=-\left(3 z^{2}+\mu^{\frac{1}{2}} z^{3}\right)+O(1), \\
K_{\mu}(z, \varphi)=2 \frac{f_{3}(\mu)}{f_{1}(\mu)} z+\mu^{\frac{1}{2}} \frac{f_{3}(\mu)}{f_{1}(\mu)} z^{2}+\mu^{\frac{1}{2}} O(1) e \\
\theta_{1}(\mu)=\theta(\mu)+\mu \frac{f_{3}(\mu)}{f_{1}(\mu)^{\prime}}
\end{gathered}
$$

então $\Phi_{\mu}$ pode ser reescrita como:

$$
\Phi_{\mu}:(z, \varphi) \mapsto\left((1-2 \mu) z+\mu^{\frac{3}{2}} H_{\mu}(z, \varphi), \varphi+\theta_{1}(\mu)+\mu^{\frac{3}{2}} K_{\mu}(z, \varphi)\right)
$$

Temos que as funções $H_{\mu}$ e $K_{\mu}$ são funções de classe $C^{4}$ em $z, \varphi$ e $\mu$, com $-1 \leq z \leq 1,0 \leq \varphi \leq 2 \pi$ e $0<\mu \leq \mu_{0}$. Para algum $\mu_{0}$ suficientemente pequeno e positivo, a região $-1 \leq z \leq 1,0 \leq \varphi \leq 2 \pi$ corresponde ao anel de largura $O(\mu)$ (nas coordenadas originais $(r, \varphi)$ ) sobre a circunferência invariante para $N \Phi_{\mu}$, que tem raio $O(\sqrt{\mu})$. Iremos construir uma curva fechada invariante para $\Phi_{\mu}$ dentro desse anel.

Antes de prosseguir com a demonstração, observemos que o comportamento qualitativo de $\Phi_{\mu}$ é agora fácil de ver. De fato $\Phi_{\mu}$ pode ser escrito como:

$$
(z, \varphi) \mapsto\left((1-2 \mu) z, \varphi+\theta_{1}(\mu)\right)
$$

mais uma perturbação pequena em $\mu$. A primeira coordenada de $\Phi_{\mu}$ é uma contração na direção de $z$. Notemos contudo, que a intensidade da contração vai a zero da ordem $\mu$, então a primeira coordenada de $\Phi_{\mu}$ se aproxima da primeira coordenada de $N \Phi_{\mu}$. Se isto não ocorresse, como $N \Phi_{\mu}$ possui uma curva invariante, e a primeira coordenada de $\Phi_{\mu}$ se aproxima da primeira coordenada de $N \Phi_{\mu}$, poderiamos utilizar resultados conhecidos sobre persistência de curvas invariantes atratoras sob uma pequena pertubação, para garantir a existência.

Passo 3: Neste passo vamos mostrar que, para $f_{1}(0)>0$ e para todo $\mu$ positivo suficientemente pequeno, $\Phi_{\mu}$ terá uma curva invariante.

A idéia é: procurar uma variedade invariante para $\Phi_{\mu}$, da forma $\{(z, \varphi) / z=u(\varphi), \varphi \in \mathbb{R}\}$, onde:

i) $u(\varphi)$ é periódica em $\varphi$, com período $2 \pi$,

ii) $|u(\varphi)| \leq 1$, para todo $\varphi$,

iii) $u(\varphi)$ é Lipschitz contínua, com constante de Lipschitz 1. 
O espaço de todas as funções $u$ satisfazendo i), ii) e iii) será denotado por $\mathcal{U}$, onde $\mathcal{U}$ é um espaço de Banach com a norma do sup. A demonstração será baseada em um teorema do ponto fixo para contrações. Em linhas gerais, o argumento será o seguinte: iniciamos com uma variedade $M=\{(z, \varphi) / z=u(\varphi), u \in \mathcal{U}, \varphi \in \mathbb{R}\}$ e consideramos a nova variedade $\Phi_{\mu} M$, obtida pela aplicação de $\Phi_{\mu}$ sobre M. Será mostrado que, para $\mu$ suficientemente pequeno, $\Phi_{\mu} M$ novamente terá a forma $\{(z, \varphi) / z=\hat{u}(\varphi), \hat{u} \in \mathcal{U}, \varphi \in \mathbb{R}\}$, e então construiremos uma aplicação não linear

$$
\begin{aligned}
\mathcal{F}: \mathcal{U} & \longrightarrow \mathcal{u} \\
u & \longmapsto \hat{u} .
\end{aligned}
$$

Provaremos então que, para $\mu$ pequeno positivo, $\mathcal{F}$ é uma contração em $\mathcal{U}$ (em relação à norma do sup), e portanto tem um único ponto fixo $u^{*}$. A variedade $\left\{z=u^{*}(\varphi)\right\}$ será a curva fechada invariante desejada. Como consequência da demonstração de contratividade será provado que essa variedade é atratora, no seguinte sentido; tomando um ponto de partida $(z, \varphi)$, com $|z| \leq 1$, e denotando $\left(z_{n}, \varphi_{n}\right)$ por $\Phi_{\mu}^{n}(z, \varphi)$, teremos que $\lim _{n \rightarrow \infty}\left(z_{n}-u^{*}\left(\varphi_{n}\right)\right)=0$. Temos que o domínio de atração contém o anel $0<|z| \leq 1$. Para completar os argumentos acima, é necessário primeiramente construir a aplicação não linear $\mathcal{F}$. Para isto, vamos proceder da seguinte maneira:

i) Mostraremos que existe uma única $\widetilde{\varphi}$ tal que a $\varphi$-componente de $\Phi_{\mu}(u(\widetilde{\varphi}), \widetilde{\varphi})$ é $\varphi$, ou seja, uma única $\widetilde{\varphi}$ tal que

$$
\varphi=\widetilde{\varphi}+\theta_{1}(\mu)+\mu^{\frac{3}{2}} K_{\mu}(u(\widetilde{\varphi}), \widetilde{\varphi}) \quad(\bmod 2 \pi),
$$

isto é, $\varphi$ difere de $\widetilde{\varphi}+\theta_{1}(\mu)+\mu^{\frac{3}{2}} K_{\mu}(u(\widetilde{\varphi}), \widetilde{\varphi})$ por um múltiplo inteiro de $2 \pi$.

ii) Tomaremos então $\mathcal{F} u(\varphi)$ igual a z-componente de $\Phi_{\mu}(u(\widetilde{\varphi}), \widetilde{\varphi})$, ou seja,

$$
\mathcal{F} u(\varphi)=(1-2 \mu) u(\widetilde{\varphi})+\mu^{\frac{3}{2}} H_{\mu}(u(\widetilde{\varphi}), \widetilde{\varphi}) \text {. }
$$

Nas estimativas que serão feitas posteriormente, será conveniente introduzir:

$$
\beta=\sup _{\substack{0 \leq \varphi \leq 2 \pi \\-1 \leq z \leq 1 \\ \mu>0}}\left\{\left|H_{\mu}\right| \vee\left|K_{\mu}\right| \vee\left|\frac{\partial H_{\mu}}{\partial z}\right| \vee\left|\frac{\partial H_{\mu}}{\partial \varphi}\right| \vee\left|\frac{\partial K_{\mu}}{\partial z}\right| \vee\left|\frac{\partial K_{\mu}}{\partial \varphi}\right|\right\}
$$


Assim definido, $\beta$ fica limitado quando $\mu \longrightarrow 0$ (pela direita). Vale a pena observar que $H_{\mu}$ e $K_{\mu}$ são de classe $C^{4}$ no compacto $[0,2 \pi] \times[-1,1]$, logo $\beta$ está bem definido.

Devemos mostrar que a equação (2.24) tem uma única solução. Para isto, será conveniente denotar o lado direito de (2.24) temporariamente por $x(\widetilde{\varphi})$ :

$$
x(\widetilde{\varphi})=\widetilde{\varphi}+\theta_{1}(\mu)+\mu^{\frac{3}{2}} K_{\mu}(u(\widetilde{\varphi}), \widetilde{\varphi}) .
$$

Queremos mostrar que, quando $\widetilde{\varphi}$ varia no intervalo $[0,2 \pi], x(\widetilde{\varphi})$ percorre exatamente uma vez um intervalo de comprimento $2 \pi$. Vale a pena relembrar que:

- $u(\widetilde{\varphi})$ é periódica em $\widetilde{\varphi}$ com período $2 \pi$,

- $K_{\mu}(z, \widetilde{\varphi})$ é periódica em $\widetilde{\varphi}$ com período $2 \pi$.

Isso nos leva a concluir que:

$$
\begin{aligned}
x(2 \pi) & =2 \pi+\theta_{1}(\mu)+\mu^{\frac{3}{2}} K_{\mu}(u(2 \pi), 2 \pi) \\
& =\theta_{1}(\mu)+\mu^{\frac{3}{2}} K_{\mu}(u(0), 0)+2 \pi \\
& =x(0)+2 \pi .
\end{aligned}
$$

Além disso, temos que mostrar que $x$ é estritamente crescente. Tomemos $\widetilde{\varphi}_{1}$ e $\widetilde{\varphi}_{2}$ reais, tais que $\widetilde{\varphi}_{1} \leq \widetilde{\varphi}_{2}$. Então:

$$
x\left(\widetilde{\varphi}_{2}\right)-x\left(\widetilde{\varphi}_{1}\right)=\widetilde{\varphi}_{2}-\widetilde{\varphi}_{1}+\mu^{\frac{3}{2}}\left[K_{\mu}\left(u\left(\widetilde{\varphi}_{2}\right), \widetilde{\varphi}_{2}\right)-K_{\mu}\left(u\left(\widetilde{\varphi}_{1}\right), \widetilde{\varphi}_{1}\right)\right]
$$

Precisamos estimar $K_{\mu}\left(u\left(\widetilde{\varphi}_{2}\right), \widetilde{\varphi}_{2}\right)-K_{\mu}\left(u\left(\widetilde{\varphi}_{1}\right), \widetilde{\varphi}_{1}\right)$. Para isto utilizamos o Teorema do Valor Médio. Temos que existe $(\widetilde{z}, \varphi) \in[-1,1] \times[0,2 \pi]$ tal que:

$$
\begin{aligned}
\left|K_{\mu}\left(u\left(\widetilde{\varphi}_{2}\right), \widetilde{\varphi}_{2}\right)-K_{\mu}\left(u\left(\widetilde{\varphi}_{1}\right), \widetilde{\varphi}_{1}\right)\right| & =\left|\left\langle\nabla K_{\mu}(\widetilde{z}, \varphi),\left(u\left(\widetilde{\varphi}_{2}\right)-u\left(\widetilde{\varphi}_{1}\right), \widetilde{\varphi}_{2}-\widetilde{\varphi}_{1}\right)\right\rangle\right| \\
& =\left|\left\langle\frac{\partial K_{\mu}}{\partial z}(\widetilde{z}, \varphi), \frac{\partial K_{\mu}}{\partial \varphi}(\widetilde{z}, \varphi)\right),\left(u\left(\widetilde{\varphi}_{2}\right)-u\left(\widetilde{\varphi}_{1}\right), \widetilde{\varphi}_{2}-\widetilde{\varphi}_{1}\right)\right\rangle \mid \\
& =\mid \frac{\partial K_{\mu}}{\partial z}(\widetilde{z}, \varphi)\left(u\left(\widetilde{\varphi}_{2}\right)-u\left(\widetilde{\varphi}_{1}\right)+\frac{\partial K_{\mu}}{\partial \varphi}(\widetilde{z}, \varphi)\left(\widetilde{\varphi}_{2}-\widetilde{\varphi}_{1}\right) \mid\right. \\
& \leq \beta\left[\left|u\left(\widetilde{\varphi}_{2}\right)-u\left(\widetilde{\varphi}_{1}\right)\right|+\left|\widetilde{\varphi}_{2}-\widetilde{\varphi}_{1}\right|\right] \\
& \leq 2 \beta\left|\widetilde{\varphi}_{2}-\widetilde{\varphi}_{1}\right|=2 \beta\left(\widetilde{\varphi}_{2}-\widetilde{\varphi}_{1}\right) .
\end{aligned}
$$


A segunda desigualdade acima vem do fato de $u(\widetilde{\varphi})$ ser Lipschitz contínua, com constante de Lipschitz 1 e a igualdade vem do fato de termos suposto que $\widetilde{\varphi}_{1} \leq \widetilde{\varphi}_{2}$. Logo:

$$
\left|K_{\mu}\left(u\left(\widetilde{\varphi}_{2}\right), \widetilde{\varphi}_{2}\right)-K_{\mu}\left(u\left(\widetilde{\varphi}_{1}\right), \widetilde{\varphi}_{1}\right)\right| \leq 2 \beta\left(\widetilde{\varphi}_{2}-\widetilde{\varphi}_{1}\right) .
$$

Daí

$$
-2 \beta\left(\widetilde{\varphi}_{2}-\widetilde{\varphi}_{1}\right) \leq K_{\mu}\left(u\left(\widetilde{\varphi}_{2}\right), \widetilde{\varphi}_{2}\right)-K_{\mu}\left(u\left(\widetilde{\varphi}_{1}\right), \widetilde{\varphi}_{1}\right) \leq 2 \beta\left(\widetilde{\varphi}_{2}-\widetilde{\varphi}_{1}\right)
$$

e assim

$$
K_{\mu}\left(u\left(\widetilde{\varphi}_{2}\right), \widetilde{\varphi}_{2}\right)-K_{\mu}\left(u\left(\widetilde{\varphi}_{1}\right), \widetilde{\varphi}_{1}\right) \geq-2 \beta\left(\widetilde{\varphi}_{2}-\widetilde{\varphi}_{1}\right) .
$$

Portanto, pela equação (2.26) e pela estimativa obtida acima, segue que:

$$
x\left(\widetilde{\varphi}_{2}\right)-x\left(\widetilde{\varphi}_{1}\right) \geq \widetilde{\varphi}_{2}-\widetilde{\varphi}_{1}-2 \beta \mu^{\frac{3}{2}}\left(\widetilde{\varphi}_{2}-\widetilde{\varphi}_{1}\right)=\left(1-2 \beta \mu^{\frac{3}{2}}\right)\left(\widetilde{\varphi}_{2}-\widetilde{\varphi}_{1}\right) .
$$

Se $\mu>\left(\frac{1}{2 \beta}\right)^{\frac{2}{3}} \Longrightarrow\left(1-2 \beta \mu^{\frac{3}{2}}\right)>0$. Logo $x\left(\widetilde{\varphi}_{2}\right)-x\left(\widetilde{\varphi}_{1}\right)>0$ e $x$ será estritamente crescente, o que implicará que $x(\widetilde{\varphi})=\varphi(\bmod 2 \pi)$ tem uma única solução $\widetilde{\varphi}$ em $[0,2 \pi]$. A função $\widetilde{\varphi}(\varphi)$ assim definida é contínua, então é possível escrever $\widetilde{\varphi}$ em função de $\varphi$.

Agora, sejam $\widetilde{\varphi}_{1}$ e $\widetilde{\varphi}_{2}$ as únicas soluções das equações $x\left(\widetilde{\varphi}_{1}\right)=\varphi_{1}$ e $x\left(\widetilde{\varphi}_{2}\right)=\varphi_{2}$, respectivamente. Da estimativa acima, obtemos que $\widetilde{\varphi}$ é Lipschitz contínua:

$$
\begin{aligned}
x\left(\widetilde{\varphi}_{2}\right)-x\left(\widetilde{\varphi}_{1}\right) & \geq\left(1-2 \beta \mu^{\frac{3}{2}}\right)\left(\widetilde{\varphi}_{2}-\widetilde{\varphi}_{1}\right) \Longleftrightarrow \\
\varphi_{2}-\varphi_{1} & \geq\left(1-2 \beta \mu^{\frac{3}{2}}\right)\left(\widetilde{\varphi}\left(\varphi_{2}\right)-\widetilde{\varphi}\left(\varphi_{1}\right)\right) \Longleftrightarrow \\
\left|\widetilde{\varphi}\left(\varphi_{2}\right)-\widetilde{\varphi}\left(\varphi_{1}\right)\right| & \leq\left(1-2 \beta \mu^{\frac{3}{2}}\right)^{-1}\left|\varphi_{2}-\varphi_{1}\right| .
\end{aligned}
$$

Concluímos então que a definição (2.25) de $\mathcal{F} u$ faz sentido, ou seja, $\Phi_{\mu}$ leva o gráfico de $u$ em um conjunto que é gráfico de alguma função $\hat{u}$, e portanto $\mathcal{F} u$ está bem definida. Vamos verificar agora que $\mathcal{F} u \in \mathcal{U}$.

Temos que verificar duas condições:

i) $\mathcal{F} u$ é periódica em $\varphi$ com período $2 \pi$.

Sabemos que $u(\widetilde{\varphi})$ e $H_{\mu}(z, \widetilde{\varphi})$ são periódicas em $\widetilde{\varphi}$ com período $2 \pi$, e por definição $\mathcal{F} u(\varphi)=$ $(1-2 \mu) u(\widetilde{\varphi})+\mu^{\frac{3}{2}} H_{\mu}(u(\widetilde{\varphi}), \widetilde{\varphi})$. Então, se $\widetilde{\varphi}$ é tal que a $\varphi$-componente de $\Phi_{\mu}(u(\widetilde{\varphi}), \widetilde{\varphi})$ é um dado $\varphi, \operatorname{logo} \widetilde{\varphi}+2 \pi$ é tal que a $\varphi$-componente de $\Phi_{\mu}(u(\widetilde{\varphi}+2 \pi), \widetilde{\varphi}+2 \pi)$ é $\varphi(\bmod 2 \pi)$, e a 
z-componente de $\Phi_{\mu}(u(\widetilde{\varphi}+2 \pi), \widetilde{\varphi}+2 \pi)$ é igual a z-componente de $\Phi_{\mu}(u(\widetilde{\varphi}), \widetilde{\varphi})$. Portanto $\mathcal{F} u(\varphi+2 \pi)=\mathcal{F} u(\varphi), \log \mathrm{F} u$ é periódica em $\varphi$.

ii) $|\mathcal{F} u(\varphi)| \leq 1$ para todo $\varphi$.

$$
\begin{aligned}
|\mathcal{F} u(\varphi)| & =\left|(1-2 \mu) u(\widetilde{\varphi})+\mu^{\frac{3}{2}} H_{\mu}(u(\widetilde{\varphi}), \widetilde{\varphi})\right| \\
& \leq(1-2 \mu)|u(\widetilde{\varphi})|+\mu^{\frac{3}{2}}\left|H_{\mu}(u(\widetilde{\varphi}), \widetilde{\varphi})\right|,
\end{aligned}
$$

$\operatorname{como}|u(\varphi)| \leq 1$ para todo $\varphi$ e pela definição de $\beta$ :

$$
|\mathcal{F} u(\varphi)| \leq 1-2 \mu+\mu^{\frac{3}{2}} \beta,
$$

segue que $|\mathcal{F} u(\varphi)| \leq 1$ para todo $\varphi$ se tomarmos $\mu$ suficientemente pequeno para que

$$
2 \mu-\mu^{\frac{3}{2}} \beta \geq 0
$$

iii) $\mathcal{F} u(\varphi)$ é Lipschitz contínua com constante 1 .

$$
\begin{aligned}
\left|\mathcal{F} u\left(\varphi_{1}\right)-\mathcal{F} u\left(\varphi_{2}\right)\right| & =\left|(1-2 \mu) u\left(\widetilde{\varphi_{1}}\right)-(1-2 \mu) u\left(\widetilde{\varphi_{2}}\right)+\mu^{\frac{3}{2}} H_{\mu}\left(u\left(\widetilde{\varphi_{1}}\right), \widetilde{\varphi_{1}}\right)-\mu^{\frac{3}{2}} H_{\mu}\left(u\left(\widetilde{\varphi_{2}}\right), \widetilde{\varphi_{2}}\right)\right| \\
& \leq(1-2 \mu)\left|u\left(\widetilde{\varphi_{1}}\right)-u\left(\widetilde{\varphi_{2}}\right)\right|+\mu^{\frac{3}{2}}\left|H_{\mu}\left(u\left(\widetilde{\varphi_{1}}\right), \widetilde{\varphi_{1}}\right)-H_{\mu}\left(u\left(\widetilde{\varphi_{2}}\right), \widetilde{\varphi_{2}}\right)\right| \\
& \leq(1-2 \mu)\left|\widetilde{\varphi_{1}}-\widetilde{\varphi_{2}}\right|+\mu^{\frac{3}{2}} \beta\left[\left|u\left(\widetilde{\varphi_{1}}\right)-u\left(\widetilde{\varphi_{2}}\right)\right|+\left|\widetilde{\varphi_{1}}-\widetilde{\varphi_{2}}\right|\right] \\
& \leq(1-2 \mu)\left|\widetilde{\varphi_{1}}-\widetilde{\varphi_{2}}\right|+2 \mu^{\frac{3}{2}} \beta\left|\widetilde{\varphi_{1}}-\widetilde{\varphi_{2}}\right| \\
& =\left(1-2 \mu+2 \mu^{\frac{3}{2}} \beta\right)\left|\widetilde{\varphi_{1}}-\widetilde{\varphi_{2}}\right| .
\end{aligned}
$$

A segunda desigualdade acima decorre da Lipschitz continuidade de $u$, e do Teorema do Valor Intermediário. Usando a estimativa de (2.27) para $\left|\widetilde{\varphi_{1}}-\widetilde{\varphi_{2}}\right|$, obtemos:

$$
\begin{aligned}
\left|\mathcal{F} u\left(\varphi_{1}\right)-\mathcal{F} u\left(\varphi_{2}\right)\right| & \leq\left(1-2 \mu+2 \mu^{\frac{3}{2}} \beta\right)\left|\widetilde{\varphi_{1}}-\widetilde{\varphi_{2}}\right| \\
& \leq\left(1-2 \mu+2 \mu^{\frac{3}{2}} \beta\right)\left(1-2 \beta \mu^{\frac{3}{2}}\right)^{-1}\left|\varphi_{1}-\varphi_{2}\right| .
\end{aligned}
$$

Então $\mathcal{F} u$ será Lipschitz contínua com constante 1, se tomarmos:

$$
\left(1-2 \mu+2 \mu^{\frac{3}{2}} \beta\right)\left(1-2 \beta \mu^{\frac{3}{2}}\right)^{-1} \leq 1
$$


Evidentemente a desigualdade (2.29) é válida para todo $\mu$ positivo suficientemente pequeno.

Nosso próximo objetivo é provar que $\mathcal{F}$ é uma contração. Sejam $u_{1}, u_{2} \in \mathcal{U}, \varphi$ qualquer e sejam $\widetilde{\varphi_{1}}, \widetilde{\varphi_{2}}$ as soluções de:

$$
\varphi=\widetilde{\varphi_{1}}+\theta_{1}(\mu)+\mu^{\frac{3}{2}} K_{\mu}\left(u_{1}\left(\widetilde{\varphi_{1}}\right), \widetilde{\varphi_{1}}\right)
$$

e

$$
\varphi=\widetilde{\varphi_{2}}+\theta_{1}(\mu)+\mu^{\frac{3}{2}} K_{\mu}\left(u_{2}\left(\widetilde{\varphi_{2}}\right), \widetilde{\varphi_{2}}\right)
$$

respectivamente. Subtraindo as equações acima, invertendo e tomando valor absoluto, teremos:

$$
\begin{aligned}
0 & =\widetilde{\varphi_{1}}-\widetilde{\varphi_{2}}+\mu^{\frac{3}{2}}\left[K_{\mu}\left(u_{1}\left(\widetilde{\varphi_{1}}\right), \widetilde{\varphi_{1}}\right)-K_{\mu}\left(u_{2}\left(\widetilde{\varphi_{2}}\right), \widetilde{\varphi_{2}}\right)\right] \Longleftrightarrow \\
\left|\widetilde{\varphi_{1}}-\widetilde{\varphi_{2}}\right| & =\mu^{\frac{3}{2}}\left|K_{\mu}\left(u_{1}\left(\widetilde{\varphi_{1}}\right), \widetilde{\varphi_{1}}\right)-K_{\mu}\left(u_{2}\left(\widetilde{\varphi_{2}}\right), \widetilde{\varphi_{2}}\right)\right| \\
& \leq \mu^{\frac{3}{2}} \beta\left[\left|u_{1}\left(\widetilde{\varphi_{1}}\right)-u_{2}\left(\widetilde{\varphi_{2}}\right)\right|+\left|\widetilde{\varphi_{1}}-\widetilde{\varphi_{2}}\right|\right] .
\end{aligned}
$$

A última desigualdade segue do Teorema do Valor Médio e da definição de $\beta$. Agora é necessário estimar $\left|u_{1}\left(\widetilde{\varphi_{1}}\right)-u_{2}\left(\widetilde{\varphi_{2}}\right)\right|$. Temos:

$$
\begin{aligned}
\left|u_{1}\left(\widetilde{\varphi_{1}}\right)-u_{2}\left(\widetilde{\varphi_{2}}\right)\right| & =\left|u_{1}\left(\widetilde{\varphi_{1}}\right)-u_{2}\left(\widetilde{\varphi_{1}}\right)+u_{2}\left(\widetilde{\varphi_{1}}\right)-u_{2}\left(\widetilde{\varphi_{2}}\right)\right| \\
& \leq\left|u_{1}\left(\widetilde{\varphi_{1}}\right)-u_{2}\left(\widetilde{\varphi_{1}}\right)\right|+\left|u_{2}\left(\widetilde{\varphi_{1}}\right)-u_{2}\left(\widetilde{\varphi_{2}}\right)\right| \\
& \leq|| u_{1}-u_{2}||+\left|\widetilde{\varphi_{1}}-\widetilde{\varphi_{2}}\right| .
\end{aligned}
$$

Substituindo a desigualdade (2.31) em (2.30), agrupando todos os termos envolvendo $\left|\widetilde{\varphi_{1}}-\widetilde{\varphi_{2}}\right|$ à esquerda e usando que $1-2 \mu^{\frac{3}{2}}>0$, teremos:

$$
\begin{aligned}
\left|\widetilde{\varphi_{1}}-\widetilde{\varphi_{2}}\right| & \leq \mu^{\frac{3}{2}} \beta\left[\left\|u_{1}-u_{2}\right\|+2\left|\widetilde{\varphi_{1}}-\widetilde{\varphi_{2}}\right|\right] \Longleftrightarrow \\
\left|\widetilde{\varphi_{1}}-\widetilde{\varphi_{2}}\right|\left(1-2 \mu^{\frac{3}{2}} \beta\right) & \leq \mu^{\frac{3}{2}} \beta\left\|u_{1}-u_{2}\right\| \Longleftrightarrow \\
\left|\widetilde{\varphi_{1}}-\widetilde{\varphi_{2}}\right| & \leq\left(1-2 \mu^{\frac{3}{2}} \beta\right)^{-1} \mu^{\frac{3}{2}} \beta\left\|u_{1}-u_{2}\right\| .
\end{aligned}
$$


Agora, utilizando a definição de $\mathcal{F} u$ e as desigualdades (2.31) e (2.32), obtemos a estimativa:

$$
\begin{aligned}
\left|\mathcal{F} u_{1}\left(\varphi_{1}\right)-\mathcal{F} u_{2}\left(\varphi_{2}\right)\right|= & \left|(1-2 \mu) u_{1}\left(\widetilde{\varphi_{1}}\right)-(1-2 \mu) u_{2}\left(\widetilde{\varphi_{2}}\right)+\mu^{\frac{3}{2}} H_{\mu}\left(u_{1}\left(\widetilde{\varphi_{1}}\right), \widetilde{\varphi_{1}}\right)-\mu^{\frac{3}{2}} H_{\mu}\left(u_{2}\left(\widetilde{\varphi_{2}}\right), \widetilde{\varphi_{2}}\right)\right| \\
\leq & (1-2 \mu)\left|u_{1}\left(\widetilde{\varphi_{1}}\right)-u_{2}\left(\widetilde{\varphi_{2}}\right)\right|+\mu^{\frac{3}{2}}\left|H_{\mu}\left(u_{1}\left(\widetilde{\varphi_{1}}\right), \widetilde{\varphi_{1}}\right)-H_{\mu}\left(u_{2}\left(\widetilde{\varphi_{2}}\right), \widetilde{\varphi_{2}}\right)\right| \\
\leq & (1-2 \mu)\left[|| u_{1}-u_{2} \|+\left|\widetilde{\varphi_{1}}-\widetilde{\varphi_{2}}\right|\right]+\mu^{\frac{3}{2}} \beta\left[\mid\left(u_{1}\left(\widetilde{\varphi_{1}}\right)-u_{2}\left(\widetilde{\varphi_{2}}\right)|+| \widetilde{\varphi_{1}}-\widetilde{\varphi_{2}} \mid\right]\right. \\
\leq & (1-2 \mu)\left[|| u_{1}-u_{2} \|+\left|\widetilde{\varphi_{1}}-\widetilde{\varphi_{2}}\right|\right]+\mu^{\frac{3}{2}} \beta\left[|| u_{1}-u_{2} \|+2\left|\widetilde{\varphi_{1}}-\widetilde{\varphi_{2}}\right|\right] \\
\leq & (1-2 \mu)\left[\left\|u_{1}-u_{2}\right\|+\left(1-2 \mu^{\frac{3}{2}} \beta\right)^{-1} \mu^{\frac{3}{2}} \beta\left\|u_{1}-u_{2}\right\|\right]+ \\
& \mu^{\frac{3}{2}} \beta\left[\left\|u_{1}-u_{2}\right\|+2\left(1-2 \mu^{\frac{3}{2}} \beta\right)^{-1} \mu^{\frac{3}{2}} \beta\left\|u_{1}-u_{2} \mid\right\|\right] \\
= & \left\|u_{1}-u_{2}\right\|\left\{(1-2 \mu)\left[1+\mu^{\frac{3}{2}} \beta\left(1-2 \mu^{\frac{3}{2}} \beta\right)^{-1}\right]+\mu^{\frac{3}{2}} \beta\left[1+2 \mu^{\frac{3}{2}} \beta\left(1-2 \mu^{\frac{3}{2}} \beta\right)^{-1}\right]\right\} .
\end{aligned}
$$

Para simplificar a expressão acima, denotemos por $\alpha$ a expressão entre chaves, ou seja,

$$
\begin{aligned}
\alpha & :=(1-2 \mu)\left[1+\mu^{\frac{3}{2}} \beta\left(1-2 \mu^{\frac{3}{2}} \beta\right)^{-1}\right]+\mu^{\frac{3}{2}} \beta\left[1+2 \mu^{\frac{3}{2}} \beta\left(1-2 \mu^{\frac{3}{2}} \beta\right)^{-1}\right] \\
& =1-2 \mu+O\left(\mu^{\frac{3}{2}}\right) .
\end{aligned}
$$

Então podemos fazer $\alpha<1$, tomando $\mu$ pequeno o bastante. Logo:

$$
\left\|\mathcal{F} u_{1}-\mathcal{F} u_{2}\right\| \leq \alpha\left\|u_{1}-u_{2}\right\|
$$

e portanto $\mathcal{F}$ é uma contração em $\mathcal{U}$. Consequentemente, pelo Teorema 1.1 temos que $\mathcal{F}$ tem um único ponto fixo $u^{*}$. Logo, $\left\{u^{*}(\varphi), \varphi\right\}$ é uma curva invariante.

Passo 4: Neste último passo mostraremos que a variedade invariante $\left\{u^{*}(\varphi), \varphi\right\}$ é atratora.

Tomemos um ponto $(z, \varphi)$ em $|z| \leq 1$, e vamos denotar $\Phi_{\mu}(z, \varphi)$ por $\left(z_{1}, \varphi_{1}\right)$. Então:

$$
\Phi_{\mu}(z, \varphi)=\left((1-2 \mu) z+\mu^{\frac{3}{2}} H_{\mu}(z, \varphi), \varphi+\theta_{1}(\mu)+\mu^{\frac{3}{2}} K_{\mu}(z, \varphi)\right)=\left(z_{1}, \varphi_{1}\right)
$$

e

$$
\begin{aligned}
\left|z_{1}\right| & \leq(1-2 \mu)|z|+\mu^{\frac{3}{2}}\left|H_{\mu}(z, \varphi)\right| \\
& \leq 1-2 \mu+\mu^{\frac{3}{2}} \beta .
\end{aligned}
$$


Mas, por (2.28) temos que $\left|z_{1}\right| \leq 1$, ou seja, teremos que $\left(z_{1}, \varphi_{1}\right)$ está novamente contido em $|z| \leq 1$. Agora seja $\widetilde{\varphi_{1}}$ a solução da equação:

$$
\varphi_{1}=\widetilde{\varphi_{1}}+\theta_{1}(\mu)+\mu^{\frac{3}{2}} K_{\mu}\left(u^{*}\left(\widetilde{\varphi_{1}}\right), \widetilde{\varphi_{1}}\right) .
$$

Pela definição de $\varphi_{1}$ temos:

$$
\varphi_{1}=\varphi+\theta_{1}(\mu)+\mu^{\frac{3}{2}} K_{\mu}(z, \varphi) .
$$

Portanto, subtraindo as duas últimas equações acima, tomando o módulo, estimando e organizando os termos, obtemos:

$$
\begin{aligned}
\left|\widetilde{\varphi_{1}}-\varphi\right| & =\mu^{\frac{3}{2}}\left|K_{\mu}\left(u^{*}\left(\widetilde{\varphi_{1}}\right), \widetilde{\varphi_{1}}\right)-K_{\mu}(z, \varphi)\right| \\
& \leq \mu^{\frac{3}{2}} \beta\left[\left|u^{*}\left(\widetilde{\varphi_{1}}\right)-z\right|+\left|\widetilde{\varphi_{1}}-\varphi\right|\right] \\
& =\mu^{\frac{3}{2}} \beta\left[\left|u^{*}\left(\widetilde{\varphi_{1}}\right)-u^{*}(\varphi)+u^{*}(\varphi)-z\right|+\left|\widetilde{\varphi_{1}}-\varphi\right|\right] \\
& \leq \mu^{\frac{3}{2}} \beta\left[\left|u^{*}\left(\widetilde{\varphi_{1}}\right)-u^{*}(\varphi)\right|+\left|z-u^{*}(\varphi)\right|+\left|\widetilde{\varphi_{1}}-\varphi\right|\right] \\
& =\mu^{\frac{3}{2}} \beta\left[\left|z-u^{*}(\varphi)\right|+2\left|\widetilde{\varphi_{1}}-\varphi\right|\right] .
\end{aligned}
$$

Portanto

$$
\left|\widetilde{\varphi_{1}}-\varphi\right| \leq \mu^{\frac{3}{2}} \beta\left(1-2 \mu^{\frac{3}{2}} \beta\right)^{-1}\left|z-u^{*}(\varphi)\right|
$$

Agora temos:

$$
u^{*}\left(\varphi_{1}\right)=\mathcal{F} u^{*}\left(\varphi_{1}\right)=(1-2 \mu) u^{*}\left(\widetilde{\varphi_{1}}\right)+\mu^{\frac{3}{2}} H_{\mu}\left(u^{*}\left(\widetilde{\varphi_{1}}\right), \widetilde{\varphi_{1}}\right)
$$

e

$$
z_{1}=(1-2 \mu) z+\mu^{\frac{3}{2}} H_{\mu}(z, \varphi) .
$$


Subtraindo as duas últimas equações, e fazendo estimativas, teremos:

$$
\begin{aligned}
\left|z_{1}-u^{*}\left(\varphi_{1}\right)\right| & =\left|(1-2 \mu)\left(z-u^{*}\left(\widetilde{\varphi_{1}}\right)\right)+\mu^{\frac{3}{2}}\left[H_{\mu}(z, \varphi)-H_{\mu}\left(u^{*}\left(\widetilde{\varphi_{1}}\right), \widetilde{\varphi_{1}}\right)\right]\right| \\
& \leq(1-2 \mu)\left|z-u^{*}\left(\widetilde{\varphi_{1}}\right)\right|+\mu^{\frac{3}{2}} \beta\left[\left|z-u^{*}\left(\widetilde{\varphi_{1}}\right)\right|+\left|\varphi-\widetilde{\varphi_{1}}\right|\right] \\
& =\left(1-2 \mu+\mu^{\frac{3}{2}} \beta\right)\left|z-u^{*}\left(\widetilde{\varphi_{1}}\right)\right|+\mu^{\frac{3}{2}} \beta\left|\varphi-\widetilde{\varphi_{1}}\right| \\
& =\left(1-2 \mu+\mu^{\frac{3}{2}} \beta\right)\left|u^{*}\left(\widetilde{\varphi_{1}}\right)-u^{*}(\varphi)+u^{*}(\varphi)-z\right|+\mu^{\frac{3}{2}} \beta\left|\varphi-\widetilde{\varphi_{1}}\right| \\
& \leq\left(1-2 \mu+\mu^{\frac{3}{2}} \beta\right)\left[\left|u^{*}\left(\widetilde{\varphi_{1}}\right)-u^{*}(\varphi)\right|+\left|u^{*}(\varphi)-z\right|\right]+\mu^{\frac{3}{2}} \beta\left|\varphi-\widetilde{\varphi_{1}}\right| \\
& =\left(1-2 \mu+\mu^{\frac{3}{2}} \beta\right)\left[\left|\widetilde{\varphi_{1}}-\varphi\right|+\left|u^{*}(\varphi)-z\right|\right]+\mu^{\frac{3}{2}} \beta\left|\varphi-\widetilde{\varphi_{1}}\right| \\
& =\left(1-2 \mu+2 \mu^{\frac{3}{2}} \beta\right)\left|\widetilde{\varphi_{1}}-\varphi\right|+\left(1-2 \mu+\mu^{\frac{3}{2}} \beta\right)\left|z-u^{*}(\varphi)\right| \\
& \leq\left(1-2 \mu+2 \mu^{\frac{3}{2}} \beta\right)\left(1-2 \mu^{\frac{3}{2}} \beta\right)^{-1} \mu^{\frac{3}{2}} \beta\left|z-u^{*}(\varphi)\right|+\left(1-2 \mu+\mu^{\frac{3}{2}} \beta\right)\left|z-u^{*}(\varphi)\right| \\
& \leq \mu^{\frac{3}{2}} \beta\left|z-u^{*}(\varphi)\right|+\left(1-2 \mu+\mu^{\frac{3}{2}} \beta\right)\left|z-u^{*}(\varphi)\right| \\
& =\left(1-2 \mu+2 \mu^{\frac{3}{2}} \beta\right)\left|z-u^{*}(\varphi)\right| \\
& =\alpha\left|z-u^{*}(\varphi)\right| .
\end{aligned}
$$

As desigualdades acima são obtidas utilizando desigualdade triangular, o Teorema do Valor Médio, (2.29), (2.33) e (2.34). Então sabendo que $\left|z_{1}-u^{*}\left(\varphi_{1}\right)\right| \leq \alpha\left|z-u^{*}(\varphi)\right|$, é fácil ver que $\left|z_{n}-u^{*}\left(\varphi_{n}\right)\right| \leq$ $\alpha^{n}\left|z-u^{*}(\varphi)\right|$, e como $\alpha<1$, temos que $\left|z_{n}-u^{*}\left(\varphi_{n}\right)\right| \rightarrow 0$ quando $n \rightarrow \infty$. Concluímos então que a variedade invariante $\left\{u^{*}(\varphi), \varphi\right\}$ é atratora. Portanto se $f_{1}(0)>0$ e $\mu>0$, existe uma vizinhança $\mathcal{V}$ da origem e $\delta>0$, tal que para $|\mu|<\delta$ e $x_{0} \in \mathcal{V}$, o conjunto $w$-limite de $x_{0}$ pertence a uma curva invariante fechada $\Gamma(\mu)$ de classe $C^{1}$ com a origem em seu interior se, e além disso $\Gamma(0)=0$. Isto encerra a demonstração desse caso.

Caso 2: $f_{1}(0)>0$ e $\mu<0$.

Temos que $|z(\mu)| r-f_{1}(\mu) r^{3}>0 \Longleftrightarrow r^{2}<\frac{|z(\mu)|}{f_{1}(\mu)}$. Tomemos $V=\left\{(r, \varphi): r^{2}<\frac{|z(\mu)|}{f_{1}(\mu)}\right.$ e q qualquer $\}$ uma vizinhança da origem. Se $\left(r_{0}, \varphi_{0}\right) \in V$, então

$$
\begin{aligned}
\left|N \Phi\left(r_{0}, \varphi_{0}\right)\right| & =|| z(\mu)\left|r_{0}-f_{1}(\mu) r_{0}^{3}\right| \\
& \leq|z(\mu)| r_{0} \\
& =|z(\mu)|\left|\left(r_{0}, \varphi_{0}\right)\right| .
\end{aligned}
$$

Como $\mu<0$, então $|z(\mu)|<1 \Longrightarrow|z(\mu)|^{n} \longrightarrow 0$, quando $n \longrightarrow \infty$. Portanto $\left|N \Phi^{n}\left(r_{0}, \varphi_{0}\right)\right| \leq$ $|z(\mu)|^{n}\left|\left(r_{0}, \varphi_{0}\right)\right| \longrightarrow 0$ quando $n \longrightarrow \infty$. Concluímos então que, dado qualquer ponto da vizinhança 
$V$, temos que seu $w$-limite é a origem, o que conclui a demonstração deste caso.

Caso 3: $f_{1}(0)<0$ e $\mu>0$.

Seja $\Phi_{\mu}^{-1}$ a inversa de $\Phi_{\mu}$. Como $\Phi_{\mu}$ é um difeomorfismo é fácil verificar que $\Phi_{\mu}^{-1}$ satisfaz as hipóteses i),ii) e iv) do Teorema 2.2. Tomemos agora a aplicação $\Psi(\mu, x):=\Phi^{-1}(-\mu, x)$. É fácil de se verificar que $\Psi(\mu, x)$ satisfaz todas as hipóteses do Teorema 2.2. Então existe uma mudança de coordenadas de classe $C^{4}$ dependente de $\mu$, levando $\Psi$ à forma:

$$
\Psi(\mu, x)=N \Psi(\mu, x)+O\left(\|x\|^{5}\right)
$$

Com as mesmas funções $f_{1}(\mu), f_{3}(\mu)$, e $\theta(\mu)$ de classe $C^{4}$ utilizadas nos casos 1 e 2 , temos que a aplicação $N \Psi(\mu, x)$, em coordenadas polares, é dada por:

$$
\left(\begin{array}{c}
r \\
\varphi
\end{array}\right) \longmapsto\left(\begin{array}{c}
\frac{1}{|z(-\mu)|} r+\frac{f_{1}(-\mu)}{|z(-\mu)|^{4}} r^{3} \\
\varphi-\theta(-\mu)-\frac{f_{3}(-\mu)}{|z(-\mu)|^{2}} r^{2}
\end{array}\right)
$$

Como $f_{1}(0)<0$ temos $\frac{f_{1}(0)}{|z(0)|^{4}}<0$ e, portanto, o coeficiente de $r^{3}$ em $N \Psi(\mu, x)$ é negativo em $\mu=0$. Logo, pelo caso 1 , existe uma vizinhança $\mathcal{V}$ da origem e $\delta>0$, tal que para $|\mu|<\delta$ e $x_{0} \in \mathcal{V}$, o conjunto $w$-limite de $x_{0}$ pertence a uma curva invariante fechada $\Gamma(\mu)$ de classe $C^{1}$ com a origem em seu interior e, além disso, $\Gamma(0)=0$. Como estamos tomando $-\mu<0$ e $\Psi(\mu, x):=\Phi^{-1}(-\mu, x)$, podemos concluir que para $\mu<0$ a aplicação $\Phi(\mu, x)$ tem $\alpha$-limite pertence a uma curva invariante fechada $\Gamma(\mu)$ de classe $C^{1}$ com a origem em seu interior e, além disso, $\Gamma(0)=0$. Isto finaliza a demonstração deste item.

Caso 4: $f_{1}(0)<0$ e $\mu<0$.

Temos que $\frac{1}{|z(-\mu)|} r+\frac{f_{1}(-\mu)}{|z(-\mu)|^{4}} r^{3}>0 \Leftrightarrow r^{2}<\frac{|z(-\mu)|^{3}}{\left|f_{1}(-\mu)\right|}$. Tomemos $V=\left\{(r, \varphi): r^{2}<\frac{|z(-\mu)|^{3}}{\left|f_{1}(-\mu)\right|}\right.$ e $\varphi$ qualquer $\}$ uma vizinhança da origem. Seja $\left(r_{0}, \varphi_{0}\right) \in V$. Utilizando a mesma aplicação $\Psi(\mu, x)$ do caso 3 , e 
tomando $\left(r_{0}, \varphi_{0}\right) \in V$, teremos:

$$
\begin{aligned}
\left|N \Psi\left(r_{0}, \varphi_{0}\right)\right| & =\left|\frac{1}{|z(-\mu)|} r_{0}+\frac{f_{1}(-\mu)}{|z(-\mu)|^{4}} r_{0}^{3}\right| \\
& \leq \frac{1}{|z(-\mu)|} r_{0} \\
& =\frac{1}{|z(-\mu)|}\left|\left(r_{0}, \varphi_{0}\right)\right| .
\end{aligned}
$$

Como $-\mu>0$, então $|z(-\mu)|>1 \Longrightarrow \frac{1}{|z(-\mu)|^{n}} \longrightarrow 0$, quando $n \longrightarrow \infty$. Portanto $\left|N \Psi^{n}\left(r_{0}, \varphi_{0}\right)\right| \leq$ $\frac{1}{|z(-\mu)|^{n}}\left|\left(r_{0}, \varphi_{0}\right)\right| \longrightarrow 0$ quando $n \longrightarrow \infty$. Concluímos então que, dado qualquer $\left(r_{0}, \varphi_{0}\right) \in V \mathrm{e} \mu<0$, a origem é o $w$-limite de qualquer ponto dessa vizinhança, para a aplicação $\Psi(\mu, x)=\Phi^{-1}(-\mu, x)$. Portanto, concluímos que para $\mu>0$ a origem é o $\alpha$-limite de qualquer ponto de $V$, para a aplicação $\Phi(\mu, x)$. Esses 4 casos finalizam a demonstração do Teorema 2.2 .

Observação: O sinal de $f_{1}(0)$ do termo cúbico é o que caracteriza a bifurcação: quando $f_{1}(0)>0$ a bifurcação é dita supercrítica, quando $f_{1}(0)<0$ é dita subcrítica. A situação no caso $f_{1}(0)=0$ não pode ser determinado apenas pelo termo cúbico.

Para o próximo capítulo de aplicações será importante obtermos uma expressão para o cálculo de $f_{1}(0)$. A proposição a seguir nos fornecerá essa expressão.

Proposição 2.1 Seja $N \Phi_{0}:(r, \varphi) \mapsto\left(r-f_{1}(0) r^{3}, \varphi+\theta(0)+f_{3}(0) r^{2}\right)$ a forma canônica aproximada para $\Phi_{0}$ dada em (2.23). Então $f_{1}(0)$ é dado por:

$$
\operatorname{Re}\left[\frac{(1-2 \lambda) \bar{\lambda}^{2}}{1-\lambda} \xi_{11} \xi_{20}\right]+\frac{1}{2}\left|\xi_{11}^{2}\right|+\left|\xi_{02}^{2}\right|-\operatorname{Re}\left[\bar{\lambda} \xi_{21}\right]
$$

onde $\lambda=\lambda(0)=e^{i \theta(0)}, e \xi_{11}, \xi_{20}, \xi_{02}$ e $\xi_{21}$ são dados como no Lema 2.1 e calculados em $\mu=0$.

Demonstração: Já vimos pelo Lema 2.1 que $f_{1}(\mu)=-\operatorname{Re}\left(\frac{\alpha_{2}^{3}}{e^{i \theta(\mu)}}\right)$ e que $\lambda(\mu)=(1+\mu) e^{i \theta(\mu)}, \operatorname{logo}$ $f_{1}(0)=-\operatorname{Re}\left(\bar{\lambda} \alpha_{2}^{3}\right)$. Observando que o coeficiente de $z^{\prime 2} \overline{z^{\prime}}$ não foi alterado pela mudança de coordenadas usada no Lema 2.3, basta determinar o coeficiente de $z^{\prime 2} \overline{z^{\prime}}$ no Lema 2.2 . O coeficiente de 
$z^{\prime 2} \overline{z^{\prime}}$ na equação (2.9) é:

$$
\lambda\left[2 \gamma_{2} \gamma_{1}+\gamma_{1}\left(\gamma_{2}+\overline{\gamma_{1}}\right)+2 \gamma_{0} \overline{\gamma_{0}}\right]+\xi_{20}\left[-2 \gamma_{1}\right]+\xi_{11}\left[-\overline{\gamma_{1}}-\gamma_{2}\right]+\xi_{02}\left[-2 \overline{\gamma_{0}}\right]+\xi_{21},
$$

e na equação (2.10) é:

$$
\gamma_{2}\left[2 \lambda\left(-\lambda \gamma_{1}+\xi_{11}\right)\right]+\gamma_{1}\left[\lambda\left(-\overline{\lambda \gamma_{1}}+\bar{\xi}_{11}\right)+\bar{\lambda}\left(-\lambda \gamma_{2}+\xi_{20}\right)\right]+\gamma_{0}\left[2 \bar{\lambda}\left(-\overline{\lambda \gamma_{0}}+\bar{\xi}_{02}\right)\right]
$$

Logo, o coeficiente de $z^{\prime 2} \overline{z^{\prime}}$ da equação (2.12) será dado pela expressão:

$$
\begin{aligned}
\alpha_{2}^{3}= & \lambda\left[2 \gamma_{2} \gamma_{1}+\gamma_{1}\left(\gamma_{2}+\overline{\gamma_{1}}\right)+2 \gamma_{0} \overline{\gamma_{0}}\right]+\xi_{20}\left[-2 \gamma_{1}\right]+\xi_{11}\left[-\overline{\gamma_{1}}-\gamma_{2}\right]+\xi_{02}\left[-2 \overline{\gamma_{0}}\right]+\xi_{21}+ \\
& \gamma_{2}\left[2 \lambda\left(-\lambda \gamma_{1}+\xi_{11}\right)\right]+\gamma_{1}\left[\lambda\left(-\overline{\lambda \gamma_{1}}+\bar{\xi}_{11}\right)+\bar{\lambda}\left(-\lambda \gamma_{2}+\xi_{20}\right)\right]+\gamma_{0}\left[2 \bar{\lambda}\left(-\overline{\lambda \gamma_{0}}+\bar{\xi}_{02}\right)\right] .
\end{aligned}
$$

Então, substituindo $\gamma_{2}, \gamma_{1}$ e $\gamma_{0}$ como determinado no Lema 2.2, teremos que $\alpha_{2}^{3}$ é dado por:

$$
\begin{aligned}
\alpha_{2}^{3}= & \lambda\left[\frac{-3 \xi_{20} \xi_{11}}{\lambda(1-\lambda)^{2}}+\frac{\left|\xi_{11}\right|^{2}}{(\lambda-1)(\bar{\lambda}-1)}+\frac{2\left|\xi_{02}\right|^{2}}{\left(\lambda-\bar{\lambda}^{2}\right)\left(\bar{\lambda}-\lambda^{2}\right)}\right]+\frac{(1-2 \lambda) \xi_{20} \xi_{11}}{\lambda(\lambda-1)}-\frac{\left|\xi_{11}\right|^{2}}{\bar{\lambda}-1} \\
& -\frac{2\left|\xi_{02}\right|^{2}}{\bar{\lambda}-\lambda^{2}}+\xi_{21}+\frac{2 \xi_{20} \xi_{11}}{(1-\lambda)^{2}}-\frac{\lambda\left|\xi_{11}\right|^{2}}{(\lambda-1)(\bar{\lambda}-1)}+\frac{\xi_{20} \xi_{11}}{(1-\lambda)^{2}}-\frac{2 \lambda\left|\xi_{02}\right|^{2}}{\left(\lambda-\bar{\lambda}^{2}\right)\left(\bar{\lambda}-\lambda^{2}\right)} \\
= & \frac{(2 \lambda-1)}{(1-\lambda) \lambda} \xi_{20} \xi_{11}+\left(\frac{-\lambda}{1-\lambda}\right)\left|\xi_{11}\right|^{2}+\left(\frac{-2 \lambda}{1-\lambda^{3}}\right)\left|\xi_{02}\right|^{2}+\xi_{21} .
\end{aligned}
$$

Mas, como $f_{1}(0)=-\operatorname{Re}\left(\bar{\lambda} \alpha_{2}^{3}\right)$, obtemos:

$$
\begin{aligned}
f_{1}(0) & =-\operatorname{Re}\left[\frac{(2 \lambda-1) \bar{\lambda}}{(1-\lambda) \lambda} \xi_{20} \xi_{11}+\left(\frac{-\lambda \bar{\lambda}}{1-\lambda}\right)\left|\xi_{11}\right|^{2}+\left(\frac{-2 \lambda \bar{\lambda}}{1-\lambda^{3}}\right)\left|\xi_{02}\right|^{2}+\bar{\lambda} \xi_{21}\right] \\
& =\operatorname{Re}\left[\frac{(1-2 \lambda) \bar{\lambda}^{2}}{(1-\lambda)} \xi_{20} \xi_{11}\right]+\operatorname{Re}\left(\frac{1}{1-\lambda}\right)\left|\xi_{11}\right|^{2}+2 \operatorname{Re}\left(\frac{1}{1-\lambda^{3}}\right)\left|\xi_{02}\right|^{2}-\operatorname{Re}\left(\bar{\lambda} \xi_{21}\right) .
\end{aligned}
$$

Sabemos que $\lambda=e^{i \theta(0)}=\cos \theta(0)+i \operatorname{sen} \theta(0)$. Então:

$$
\begin{aligned}
& \frac{1}{1-\lambda}=\frac{1}{2}+i \frac{\operatorname{sen} \theta(0)}{2(1-\cos \theta(0))} \quad \Longrightarrow \quad \operatorname{Re}\left(\frac{1}{1-\lambda}\right)=\frac{1}{2} \quad \mathrm{e} \\
& \frac{1}{1-\lambda^{3}}=\frac{1}{2}+i \frac{\operatorname{sen} 3 \theta(0)}{2(1-\cos 3 \theta(0))} \quad \Longrightarrow \operatorname{Re}\left(\frac{1}{1-\lambda^{3}}\right)=\frac{1}{2}
\end{aligned}
$$


Portanto, substituindo as duas últimas equações em (2.36), teremos finalmente:

$$
f_{1}(0)=\operatorname{Re}\left[\frac{(1-2 \lambda) \bar{\lambda}^{2}}{(1-\lambda)} \xi_{11} \xi_{20}\right]+\frac{1}{2}\left|\xi_{11}\right|^{2}+\left|\xi_{02}\right|^{2}-\operatorname{Re}\left(\bar{\lambda} \xi_{21}\right) .
$$




\section{CAPÍTULO 3}

\section{Uma Aplicação.}

Neste capítulo faremos uma aplicação do Teorema de Bifurcação de Poincaré-Andronov-Hopf para difeomorfismo do plano, abordado no capítulo anterior. Analisaremos propriedades de estabilidade das soluções de equilíbrio e a existência de curvas invariantes fechadas de um sistema dinâmico no plano, cujas órbitas imitam a evolução do preço de uma mercadoria e do excesso de demanda por essa mercadoria. O sistema aqui estudado provem de um modelo estocástico, cuja construção pode ser vista em [3]. Os parâmetros do sistema correspondem às seguintes variáveis do mercado: (a) a proporção de especuladores, (b) a tendência dos operadores especulativos, (c) o grau de heterogeneidade das avaliações dos agentes de mercado em relação ao valor fundamental da mercadoria; e (d) a força de influência do excesso de demanda da população sobre a variação do preço da mercadoria. Esta correspondência permite empregar os resultados para inferir causas plausíveis para as flutuações de preços e demanda em um mercado de ativos reais.

O emprego de sistemas dinâmicos para estudar a evolução de modelos estocásticos de fenômenos sócio-econômico é bastante comum na área de modelos ativo de interação heterogênea. No entanto, na grande maioria dos casos presentes na literatura, esses sistemas dinâmicos são unidimensionais. Ao final desse capítulo faremos uma simulação numérica, para mostrar o surgimento de oscilações periódicas por variação de um parâmetro. 


\subsection{Introdução.}

Investigaremos o equilíbrio e as propriedades de estabilidade da origem no sistema dinâmico discreto gerado pela aplicação $\Phi$ no plano, dada por:

$$
\left(\begin{array}{c}
p_{n} \\
d_{n}
\end{array}\right)=\Phi\left(\begin{array}{c}
p_{n-1} \\
d_{n-1}
\end{array}\right)=\left(\begin{array}{c}
p_{n-1}+\lambda d_{n-1} \\
\alpha\left[1-2 \Psi\left(p_{n-1}+(\lambda-J) d_{n-1}\right)\right]+(1-\alpha)\left[1-2 \Psi\left(p_{n-1}+\lambda d_{n-1}\right)\right]
\end{array}\right) .
$$

No sistema dinâmico acima, $\alpha \in(0,1), \lambda$ e $J$ são números reais positivos, e $\Psi$ é uma função de distribuição de probabilidade. Uma vez que não impomos restrições rígidas sobre a função de distribuição de probabilidade, a análise de (3.1) se torna uma tarefa não trivial. O modelo foi construído com o objetivo de investigar a evolução de duas características particulares: os preços das mercadorias e o excesso de demanda da população por uma mercadoria no mercado mundial real. No modelo abordado, as evoluções de interesse são representadas por processos estocásticos. Estes processos possuem as seguintes propriedades: como um modelo de população agente crescente, suas trajetórias convergem para órbitas do sistema dinâmico (3.1). Essa convergência gera a seguinte interpretação dos parâmetros e variáveis de (3.1), em termos de um único mercado de risco:

$p_{n}$ corresponde ao preço de mercado de ativos no tempo $n$;

$d_{n}$ corresponde ao excesso de demanda da população para o ativo no tempo $n$;

$\alpha$ corresponde a proporção de especulação entre os operadores de mercado;

$J$ corresponde a tendência especulativa dos operadores;

$\lambda$ corresponde a resposta do excesso de demanda no incremento da atualização do preço do ativo;

$\Psi$ corresponde a distribuição dos desvios em torno de uma constante $\bar{v}$ das avaliações individuais do valor do ativo fundamental, onde $\bar{v}$ corresponde ao mercado de valor fundamental do ativo. Temos também que $\Psi$ satisfaz as seguintes condições:

a) $\Psi$ é diferenciável em todo domínio, cuja derivada denotaremos por $\Psi^{\prime}$;

b) $\sup _{x}\left\{\Psi^{\prime}(x)\right\}=\Psi^{\prime}(0)$;

c) $\Psi^{\prime}$ é estritamente crescente em $(-\infty, 0)$, e estritamente decrescente em $(0, \infty)$;

d) $\Psi(0)=\frac{1}{2}$. 
Os resultados do estudo do sistema dinâmico (3.1) são dados pelos Teoremas 3.1 e 3.2 das próximas seções. O Teorema 3.1 afirma que o ponto $(0,0)$ é o único ponto de equilíbrio de (3.1), e determina quando o mesmo é localmente assintoticamente estável ou instável. Uma característica interessante desse teorema é que caracteriza a estabilidade / instabilidade através de uma relação entre duas expressões envolvendo apenas os parâmetros do modelo (que são três números reais e uma função de distribuição de probabilidade). Essa redução do espaço dos parâmetros nos permite apresentar o diagrama de fases da figura (3.1), representando a estabilidade/instabilidade do ponto de equilíbrio. O segundo resultado, o Teorema 3.2, dá condições sobre os valores de parâmetro para o aparecimento de uma curva invariante fechada estável do sistema dinâmico.

\subsection{A Unicidade do Equilíbrio e Propriedades Básicas de Estabilidade.}

Teorema 3.1 (Unicidade do equilíbrio e sua estabilidade).

a) A origem, isto é, o ponto $(0,0)$ é o único equilíbrio do sistema dinâmico (3.1).

b) Se $\lambda \Psi^{\prime}(0)>1+2 \alpha J \Psi^{\prime}(0)$, então a origem é um equilíbrio instável de (3.1). Se $\lambda \Psi^{\prime}(0) \leq 1+$ $2 \alpha J \Psi^{\prime}(0)$, então a estabilidade da origem depende do valor de $2 \alpha J \Psi^{\prime}(0)$ da seguinte maneira:

- Se $2 \alpha J \Psi^{\prime}(0)<1$, então a origem é localmente assintoticamente estável;

- Se $2 \alpha J \Psi^{\prime}(0)>1$, então a origem é instável.

\section{Demonstração:}

a) Usando o fato que $\Psi(0)=\frac{1}{2}$, verifica-se facilmente que a origem é ponto fixo de $\Phi$. Suponhamos agora que o ponto $\left(p_{0}, d_{0}\right)$ é um ponto fixo de $\Phi$. Então $\Phi\left(p_{0}, d_{0}\right)=\left(p_{0}, d_{0}\right)$, ou seja,

$$
\left(\begin{array}{l}
p_{0} \\
d_{0}
\end{array}\right)=\left(\begin{array}{c}
p_{0}+\lambda d_{0} \\
\alpha\left[1-2 \Psi\left(p_{0}+(\lambda-J) d_{0}\right)\right]+(1-\alpha)\left[1-2 \Psi\left(p_{0}+\lambda d_{0}\right)\right]
\end{array}\right) .
$$

Da primeira equação acima, temos que $\lambda d_{0}=0$, o que implica que $d_{0}=0$, pois $\lambda>0$. Substituindo $d_{0}=0$ na segunda equação acima, teremos que $1-2 \Psi\left(p_{0}\right)=0 \Rightarrow \Psi\left(p_{0}\right)=\frac{1}{2}$. Daí teremos que $p_{0}=0$, pois $\Psi$ é monótona e $\Psi(0)=\frac{1}{2}$. Portanto, $\left(p_{0}, d_{0}\right)=(0,0)$, o que completa a demonstração deste item. 
b) Temos que a matriz jacobiana de $\Phi$ calculada na origem é dada por:

$$
D \Phi(0,0)=\left(\begin{array}{cc}
1 & \lambda \\
-2 \Psi^{\prime}(0) & 2(\alpha J-\lambda) \Psi^{\prime}(0)
\end{array}\right) .
$$

O polinômio característico é dado por:

$$
p(z)=z^{2}-\left[1+2(\alpha J-\lambda) \Psi^{\prime}(0)\right] z+2 \alpha J \Psi^{\prime}(0)
$$

com raízes dadas por:

$$
\begin{aligned}
& z_{1}=\frac{1}{2}\left[1+2(\alpha J-\lambda) \Psi^{\prime}(0)+\sqrt{\Delta}\right] \mathrm{e} \\
& z_{2}=\frac{1}{2}\left[1+2(\alpha J-\lambda) \Psi^{\prime}(0)-\sqrt{\Delta}\right]
\end{aligned}
$$

onde

$$
\Delta:=\left[1+2(\alpha J-\lambda) \Psi^{\prime}(0)\right]^{2}-8 \alpha J \Psi^{\prime}(0)
$$

As afirmações deste item do teorema seguirão do estado da linearização de (3.1) em torno da origem, via análise dos valores de $z_{1}$ e $z_{2}$. A análise será dividida em seis casos, correspondentes às regiões indicadas na figura 3.1. Esta figura é um tipo de diagrama de fases em duas dimensões. Devido ao comportamento das raízes, a análise da estabilidade dependerá apenas da relação entre as expressões $2 \alpha J \Psi^{\prime}(0)$ e $2 \lambda \Psi^{\prime}(0)$. Durante todos os casos a seguir, usaremos nos cálculos o fato de que $\lambda>0$ (a restrição sobre $\lambda$, imposta por construção) e que $\Psi^{\prime}(0)>0$ (essa desigualdade segue das condições a), b) e c) sobre $\Psi$ ).

Caso 1: $\Delta \leq 0$ (região 1, na figura 1)

Neste caso teremos duas raízes complexas conjugadas simples, com parte real e imaginária dadas por:

$$
\operatorname{Re}\left(z_{1}\right)=\operatorname{Re}\left(z_{2}\right)=\frac{1}{2}\left[1+2(\alpha J-\lambda) \Psi^{\prime}(0)\right]
$$

e

$$
\operatorname{Im}\left(z_{1}\right)=-\operatorname{Im}\left(z_{2}\right)=\frac{\sqrt{-\Delta}}{2} .
$$




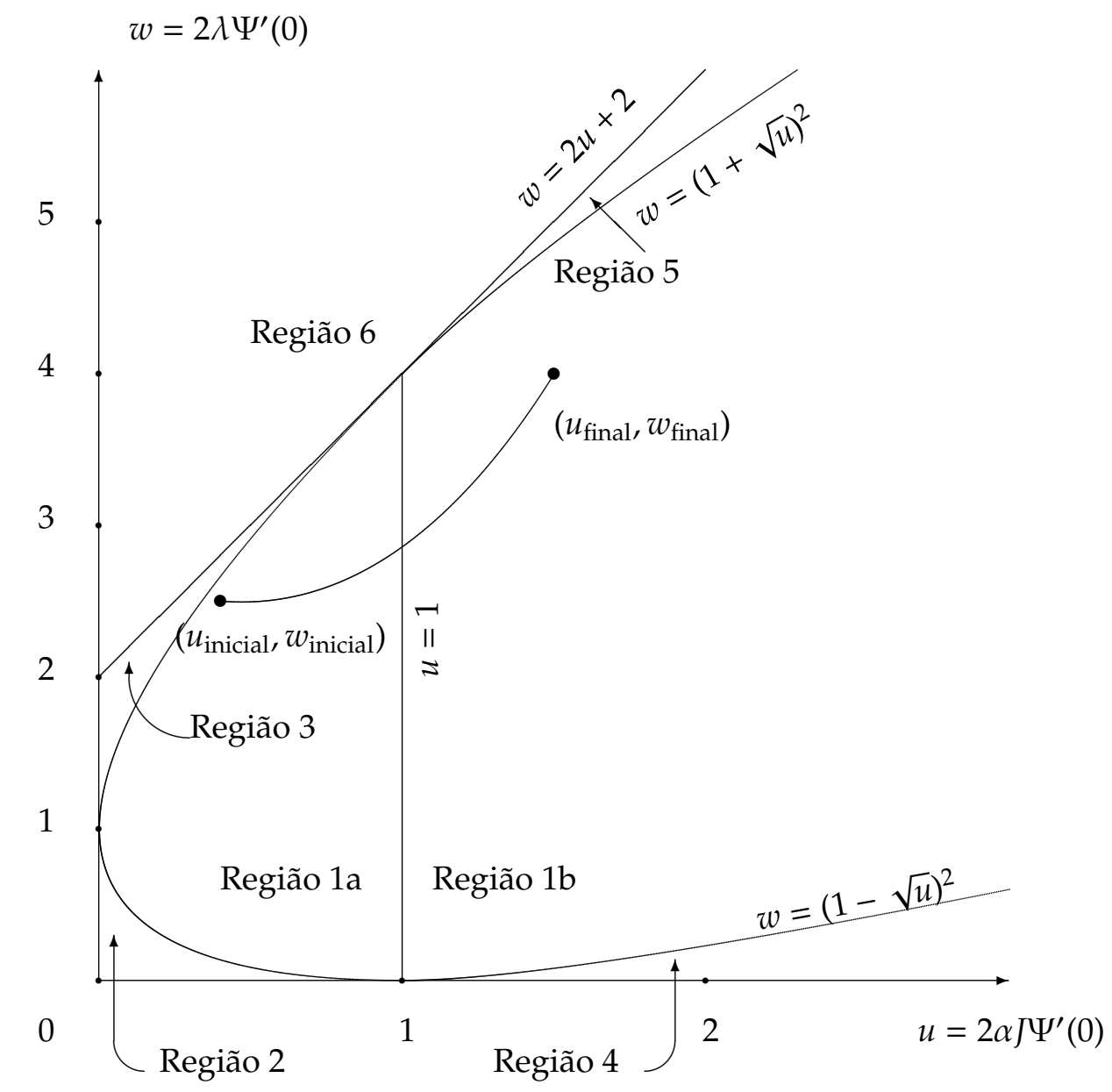

Figura 3.1: Diagrama de fase que representa a estabilidade do único ponto de equilíbrio do sistema dinâmico (3.1). 
Então

$$
\left|z_{1}\right|^{2}=\left|z_{2}\right|^{2}=\frac{1}{4}\left[1+2(\alpha J-\lambda) \Psi^{\prime}(0)\right]^{2}+\frac{\sqrt{(-\Delta)^{2}}}{4}=2 \alpha J \Psi^{\prime}(0),
$$

e, portanto, a origem é localmente assintóticamente estável no caso em que $2 \alpha J \Psi^{\prime}(0)<1$ (região 1a), e instável no caso em que $2 \alpha J \Psi^{\prime}(0)>1$ (região $1 b$ ).

Caso 2: $\Delta>0,2 \lambda \Psi^{\prime}(0)<1+2 \alpha J \Psi^{\prime}(0)$ e $2 \alpha J \Psi^{\prime}(0)<1$ (região 2, na figura 1).

Sabendo que $\lambda>0, \Psi^{\prime}(0)>0$ e das hipóteses, temos que $0<1+2(\alpha J-\lambda) \Psi^{\prime}(0)<1+2 \alpha J \Psi^{\prime}(0)$. Por adição e subtração de $\left[1+2 \alpha J \Psi^{\prime}(0)\right]^{2}$ na expressão de $\Delta$, obtemos:

$$
\begin{aligned}
\Delta & =\left[1+2 \alpha J \Psi^{\prime}(0)\right]^{2}-8 \alpha J \Psi^{\prime}(0)+\left[1+2(\alpha J-\lambda) \Psi^{\prime}(0)\right]^{2}-\left[1+2 \alpha J \Psi^{\prime}(0)\right]^{2} \\
& =\left[1-2 \alpha J \Psi^{\prime}(0)\right]^{2}+\left[1+2(\alpha J-\lambda) \Psi^{\prime}(0)\right]^{2}-\left[1+2 \alpha J \Psi^{\prime}(0)\right]^{2} \\
& <\left[1-2 \alpha J \Psi^{\prime}(0)\right]^{2}
\end{aligned}
$$

pois $\left[1+2(\alpha J-\lambda) \Psi^{\prime}(0)\right]^{2}-\left[1+2 \alpha J \Psi^{\prime}(0)\right]^{2}<0$. Então $\sqrt{\Delta}<1-2 \alpha J \Psi^{\prime}(0)$, pois $2 \alpha J \Psi^{\prime}(0)<1$. Logo,

$$
\begin{aligned}
z_{1} & =\frac{1+2(\alpha J-\lambda) \Psi^{\prime}(0)}{2}+\frac{\sqrt{\Delta}}{2} \\
& <\frac{1+2(\alpha J-\lambda) \Psi^{\prime}(0)}{2}+\frac{1-2 \alpha J \Psi^{\prime}(0)}{2} \\
& =1-\lambda \Psi^{\prime}(0) .
\end{aligned}
$$

Por hipótese temos que:

$$
\begin{aligned}
2 \lambda \Psi^{\prime}(0) & <1+2 \alpha J \Psi^{\prime}(0) \Longleftrightarrow \\
2 \lambda \Psi^{\prime}(0) & <2 \Longleftrightarrow \\
0<\lambda \Psi^{\prime}(0) & <1 .
\end{aligned}
$$

Então temos que $0<z_{1}<1$. Logo $\left|z_{1}\right|<1$. Como $-\left[1+2(\alpha J-\lambda) \Psi^{\prime}(0)\right]$ (o coeficiente de $z$ no 
polinômio característico) é negativo, segue que:

$$
\begin{aligned}
0 & <z_{1}+z_{2} \\
& <z_{2}+1 \Longleftrightarrow \\
z_{2} & >-1 .
\end{aligned}
$$

Temos também que $z_{2}<z_{1}<1, \operatorname{logo}-1<z_{2}<1 \Longrightarrow\left|z_{2}\right|<1$. Como $\left|z_{1}\right|<1$ e $\left|z_{2}\right|<1$, a origem é localmente assintoticamente estável.

Caso 3: $\Delta>0,1+2 \alpha J \Psi^{\prime}(0)<2 \lambda \Psi^{\prime}(0)<2+4 \alpha J \Psi^{\prime}(0)$ e $2 \alpha J \Psi^{\prime}(0)<1$ (região 3, na figura 1).

Das hipóteses, segue que:

$$
\begin{aligned}
2 \lambda \Psi^{\prime}(0) & <2+4 \alpha J \Psi^{\prime}(0) \Longleftrightarrow \\
-\left[1+2 \alpha J \Psi^{\prime}(0)\right] & <1+2(\alpha J-\lambda) \Psi^{\prime}(0)<0,
\end{aligned}
$$

pois $1+2 \alpha J \Psi^{\prime}(0)<2 \lambda \Psi^{\prime}(0)$, por hipótese. Analogamente ao caso 2 , obtemos que $\sqrt{\Delta}<$ $1-2 \alpha J \Psi^{\prime}(0)$. Portanto:

$$
\begin{aligned}
z_{2} & =\frac{1+2(\alpha J-\lambda) \Psi^{\prime}(0)}{2}-\frac{\sqrt{\Delta}}{2} \\
& >\frac{1+2(\alpha J-\lambda) \Psi^{\prime}(0)}{2}-\frac{1-2 \alpha J \Psi^{\prime}(0)}{2} \\
& =\frac{4 \alpha J \Psi^{\prime}(0)-2 \lambda \Psi^{\prime}(0)}{2} \\
& =2 \alpha J \Psi^{\prime}(0)-\lambda \Psi^{\prime}(0) .
\end{aligned}
$$

Como, por hipótese, $2 \lambda \Psi^{\prime}(0)<2+4 \alpha J \Psi^{\prime}(0)$, então $2 \alpha J \Psi^{\prime}(0)-\lambda \Psi^{\prime}(0)>-1$. Logo $z_{2}>-1$. Por outro lado temos que:

$$
\begin{aligned}
z_{2} & =\frac{1+2(\alpha J-\lambda) \Psi^{\prime}(0)}{2}-\frac{\sqrt{\Delta}}{2} \\
& <\frac{1+2(\alpha J-\lambda) \Psi^{\prime}(0)}{2} \\
& <0
\end{aligned}
$$

pois, por hipótese, $1+2(\alpha J-\lambda) \Psi^{\prime}(0)<0$. Logo $-1<z_{2}<0 \Longrightarrow\left|z_{2}\right|<1$. Temos que neste caso, o 
coeficiente de $z$ no polinômio característico é positivo, e segue que $z_{1}+z_{2}<0 \Longrightarrow z_{1}<-z_{2}<1$. Por outro lado $-1<z_{2}<z_{1}$, logo temos que $\left|z_{1}\right|<1$. Como $\left|z_{1}\right|<1$ e $\left|z_{2}\right|<1$, então a origem será localmente assintoticamente estável.

Caso 4: $\Delta>0,2 \lambda \Psi^{\prime}(0)<2 \alpha J \Psi^{\prime}(0)-1$ e $2 \alpha J \Psi^{\prime}(0)>1$ (região 4, na figura 1).

Agora, temos por hipótese que $2(\alpha J-\lambda) \Psi^{\prime}(0)>1$ e $1-2 \alpha J \Psi^{\prime}(0)<0$. Logo teremos:

$$
\begin{aligned}
z_{1} & =\frac{1+2(\alpha J-\lambda) \Psi^{\prime}(0)}{2}+\frac{\sqrt{\Delta}}{2} \\
& >\frac{1}{2}+\frac{2(\alpha J-\lambda) \Psi^{\prime}(0)}{2} \\
& >1
\end{aligned}
$$

pois $2(\alpha J-\lambda) \Psi^{\prime}(0)>1$. Portanto $z_{1}>1$, e concluímos que a origem é instável.

Caso 5: $\Delta>0,1+2 \alpha J \Psi^{\prime}(0)<2 \lambda \Psi^{\prime}(0) \leq 2+4 \alpha J \Psi^{\prime}(0)$ e $2 \alpha J \Psi^{\prime}(0)>1$ (região 5, na figura 1).

Como $\Delta>0$, então $\left[1+2(\alpha J-\lambda) \Psi^{\prime}(0)\right]^{2}>8 \alpha J \Psi^{\prime}(0)$. Logo $1+2(\alpha J-\lambda) \Psi^{\prime}(0)>2 \sqrt{2 \alpha J \Psi^{\prime}(0)}$ ou $1+2(\alpha J-\lambda) \Psi^{\prime}(0)<-2 \sqrt{2 \alpha J \Psi^{\prime}(0)}$ e, consequentemente, devemos ter que $2 \lambda \Psi^{\prime}(0)<$ $\left(1-\sqrt{2 \alpha J \Psi^{\prime}(0)}\right)^{2}$ ou $2 \lambda \Psi^{\prime}(0)>\left(1+\sqrt{2 \alpha J \Psi^{\prime}(0)}\right)^{2}$. Mas

$$
\begin{aligned}
\left(1-\sqrt{2 \alpha J \Psi^{\prime}(0)}\right)^{2} & =1-2 \sqrt{2 \alpha J \Psi^{\prime}(0)}+2 \alpha J \Psi^{\prime}(0) \\
& <1+2 \alpha J \Psi^{\prime}(0) \\
& <2 \lambda \Psi^{\prime}(0) .
\end{aligned}
$$

Portanto a segunda opção é falsa. Então:

$$
\begin{aligned}
2 \lambda \Psi^{\prime}(0) & >\left(1+\sqrt{2 \alpha J \Psi^{\prime}(0)}\right)^{2} \\
& =1+2 \sqrt{2 \alpha J \Psi^{\prime}(0)}+2 \alpha J \Psi^{\prime}(0) \\
& >3+2 \alpha J \Psi^{\prime}(0)
\end{aligned}
$$


pois por hipótese $2 \alpha J \Psi^{\prime}(0)>1$. Obtemos, portanto, que $2(\alpha J-\lambda) \Psi^{\prime}(0)<-3$, e segue que:

$$
\begin{aligned}
z_{2} & =\frac{1+2(\alpha J-\lambda) \Psi^{\prime}(0)}{2}-\frac{\sqrt{\Delta}}{2} \\
& <\frac{1+2(\alpha J-\lambda) \Psi^{\prime}(0)}{2} \\
& <-1 .
\end{aligned}
$$

Portanto $z_{2}<-1$, e a origem é instável neste caso.

Caso 6: $\Delta>0,2 \lambda \Psi^{\prime}(0)>2+4 \alpha J \Psi^{\prime}(0)$ (região 6, na figura 1).

Por hipótese, segue que:

$$
\begin{aligned}
1+2(\alpha J-\lambda) \Psi^{\prime}(0) & <1+2 \alpha J \Psi^{\prime}(0)-2-4 \alpha J \Psi^{\prime}(0) \\
& =-1-2 \alpha J \Psi^{\prime}(0)<0 .
\end{aligned}
$$

Então teremos:

$$
\begin{aligned}
\Delta & =\left(1+2(\alpha J-\lambda) \Psi^{\prime}(0)\right)^{2}-8 \alpha J \Psi^{\prime}(0) \\
& >\left(1+2 \alpha J \Psi^{\prime}(0)\right)^{2}-8 \alpha J \Psi^{\prime}(0) \\
& =\left(1-2 \alpha J \Psi^{\prime}(0)\right)^{2} .
\end{aligned}
$$

Se $2 \alpha J \Psi^{\prime}(0)<1$, então $\sqrt{\Delta}>1-2 \alpha J \Psi^{\prime}(0)$. Logo obtemos:

$$
\begin{aligned}
z_{2} & =\frac{1+2(\alpha J-\lambda) \Psi^{\prime}(0)}{2}-\frac{\sqrt{\Delta}}{2} \\
& <\frac{1+2(\alpha J-\lambda) \Psi^{\prime}(0)}{2}-\frac{1-2 \alpha J \Psi^{\prime}(0)}{2} \\
& =2 \alpha J \Psi^{\prime}(0)-\lambda \Psi^{\prime}(0) \\
& <2 \alpha J \Psi^{\prime}(0)-1-2 \alpha J \Psi^{\prime}(0) \\
& =-1 .
\end{aligned}
$$


Se $2 \alpha J \Psi^{\prime}(0)>1$, então $\sqrt{\Delta}>2 \alpha J \Psi^{\prime}(0)-1$ e obtemos:

$$
\begin{aligned}
z_{2} & <\frac{1+2(\alpha J-\lambda) \Psi^{\prime}(0)}{2}-\frac{-1+2 \alpha J \Psi^{\prime}(0)}{2} \\
& =1-\lambda \Psi^{\prime}(0) \\
& <1-1-2 \alpha J \Psi^{\prime}(0) \\
& =-2 \alpha J \Psi^{\prime}(0) \\
& <-1
\end{aligned}
$$

Assim $z_{2}<-1$ em ambos os casos. Portanto a origem é instável.

\subsection{Bifurcação de Hopf e Órbitas Periódicas.}

A ocorrência de uma bifurcação de Hopf no sistema dinâmico (3.1) é o assunto do Teorema 3.2 desta seção, que é baseado no Teorema 2.2. Vimos que este teorema contém uma descrição da bifurcação de Hopf e dá condições suficientes para a sua ocorrência. Isto implica o surgimento de oscilações periódicas no sistema dinâmico (3.1), para um conjunto particular de valores dos parâmetros. O teorema a seguir mostra o aparecimento da bifurcação de Hopf no sistema dinâmico (3.1), quando são satisfeitas certas hipóteses adicionais.

Teorema 3.2 Suponha que o sistema dinâmico (3.1) satisfaz as seguintes hipóteses:

1) Os parâmetros $\lambda, \alpha, J$ e a função de distribuição $\Psi(\cdot)$ são todas funções de uma variável real $\mu$, que estão definidas em algum intervalo não degenerado $\left[\mu_{\text {inicial }}, \mu_{\text {final }}\right]$, as funções $\lambda_{\mu}, \alpha_{\mu}, J_{\mu}$ e as funções de distribuição $\left\{\Psi_{\mu}(\cdot)\right\}$ são suaves o suficiente para assegurar que $\Phi$, a aplicação que gera o sistema dinâmico (3.1), seja $C^{4}$ em $\left(p_{n-1}, d_{n-1}\right)$ e $\mu$;

2) As funções $\alpha_{\mu}, J_{\mu} e \Psi_{\mu}^{\prime}(0)$ (isto é, $\left.\left.\frac{\partial \Psi_{\mu}(x)}{\partial x}\right|_{x=0}\right)$ são todas crescentes em $\mu$ no domínio $\left[\mu_{\text {inicial }}, \mu_{\text {final }}\right]$, $\left.e \frac{\partial}{\partial \mu}\left(2 \alpha_{\mu} J_{\mu} \Psi_{\mu}^{\prime}(0)\right)\right|_{\mu=\mu_{0}}>0$

3) $\left[1+2\left(\alpha_{\mu} J_{\mu}-\lambda_{\mu}\right) \Psi_{\mu}^{\prime}(0)\right]^{2}-8 \alpha_{\mu} J_{\mu} \Psi_{\mu}^{\prime}(0)<0$, para cada $\mu \in\left[\mu_{\text {inicial }}, \mu_{\text {final }}\right]$;

4) Existe um único $\mu_{0} \in\left[\mu_{\text {inicial }}, \mu_{\text {final }}\right]$ tal que $2 \alpha_{\mu_{0}} J_{\mu_{0}} \Psi_{\mu_{0}}^{\prime}(0)=1$, com $\mu_{0} \neq \mu_{\text {inicial }}$ e $\mu_{0} \neq \mu_{\text {final }}$. 
Suponhamos ainda que:

$$
\text { a) } \left.\Psi_{\mu_{0}}^{\prime \prime}(0)=0 ; \quad b\right) \Psi_{\mu_{0}}^{\prime \prime \prime}(0)<0 .
$$

Então o sistema (3.1) passa por uma bifurcação de Hopf supercrítica quando $\mu$ passa por $\mu_{0}$.

Demonstração: Para provarmos o teorema, em virtude do Teorema 2.2 e da Proposição 2.1, é suficiente mostrar que a aplicação $\Phi$ que gera o sistema dinâmico (3.1) satisfaz as condições (i)-(iv) do Teorema 2.2, e que $f_{1}(0)>0$ (a expressão de $f_{1}$ é dada na Proposição 2.1). Para isso podemos supor, reparametrizando se for necessário, que $\mu_{0}=0$. Vamos fazer a demonstração dividindo-a nos de passos 1 a 5 abaixo:

Passo 1: Neste passo verificaremos que $\Phi$ satisfaz a hipótese i) do Teorema 2.2 .

Pelo Teorema 3.1 temos que $(0,0)$ é um ponto de equilíbrio de (3.1) para todos valores dos parâmetros desse sistema dinâmico. Portanto $\Phi$ satisfaz (i) para cada $\mu \in\left[\mu_{\text {inicial }}, \mu_{\text {final }}\right]$.

Passo 2: Neste passo verificaremos que $\Phi$ satisfaz a hipótese ii) do Teorema 2.2

Começamos recordando alguns fatos estabelecidos durante a demonstração do Teorema 3.1: a) a matriz jacobiana da aplicação $\Phi$ em $(0,0)$ foi calculada e o resultado é apresentado em (3.2); b) os autovalores foram calculados e suas expressões são apresentadas em (3.3); c) os autovalores são complexos se $\Delta<0$, com $\Delta$ definido em (3.4); d) o módulo de cada autovalor é igual a $\sqrt{2 \alpha_{0} J_{0} \Psi_{0}^{\prime}(0)}$ se $\Delta \leq 0$. Agora, pela hipótese 3), temos que $\Delta<0$ para todo $\mu$. Portanto o item c) acima implica que $\Phi$ satisfaz a primeira parte da condição (ii). Já a segunda parte dessa condição segue imediatamente das hipótese 4 e do item d) acima.

Passo 3: Neste passo verificaremos que $\Phi$ satisfaz a hipótese iii) do Teorema 2.2.

O item d) do passo 2 e as hipóteses 2 e 3 implicam imediatamente que a condição (iii) é satisfeita para os autovalores de $\Phi$ em $\mu_{0}=0$.

Passo 4: Neste passo verificaremos que $\Phi$ satisfaz a hipótese iv) do Teorema 2.2.

Do item b) do passo 2, e da hipótese 3 segue que:

$$
z_{1}(0)=\frac{1+2\left(\alpha_{0} J_{0}-\lambda_{0}\right) \Psi_{0}^{\prime}(0)}{2}+i \frac{\sqrt{8 \alpha_{0} J_{0} \Psi_{0}^{\prime}(0)-\left[1+2\left(\alpha_{0} J_{0}-\lambda_{0}\right) \Psi_{0}^{\prime}(0)\right]^{2}}}{2} .
$$

Usando a hipótese 4 na expressão em (3.6) , segue que $z_{1}(0)=1-\lambda_{0} \Psi_{0}^{\prime}(0)+i \sqrt{2 \lambda_{0} \Psi_{0}^{\prime}(0)-\lambda_{0}^{2} \Psi_{0}^{\prime}(0)^{2}}$. Desse fato, temos que $z_{1}^{k}(0)=1$ para $k=1,2,3,4$ e 5 , apenas se $\lambda_{0} \Psi_{0}^{\prime}(0)=0$. Mas, pelas hipóteses 3) e 4) isso não é possível. Portanto $z_{1}^{k}(0) \neq 1$ para $k=1,2,3,4$ e 5 , provando assim a validade da condição (iv). 
Passo 5: Neste passo iremos provar que $f_{1}(0)>0$, o que implicará a bifurcação de Hopf.

Omitiremos a dependência de $\mu_{0}=0$ ao longo da demonstração deste passo, isto é, denotaremos $f_{1}(0), \lambda_{0}, \alpha_{0}, J_{0}$ e $\Psi_{0}^{\prime}(0)$ por $f_{1}, \lambda, \alpha, J$ e $\Psi^{\prime}(0)$ respectivamente. Como nossa intenção é usar a expressão (2.35), necessitamos obter uma forma como (2.1) para a aplicação $\Phi$. Vamos começar pela matriz jacobiana avaliada em $(0,0)$ da aplicação $\Phi$, que já foi calculada na demonstração do Teorema 3.1 (a matriz é representada em (3.2) ). Pela hipótese 4 temos que $2 \alpha J \Psi^{\prime}(0)=1$. Assim a matriz adquire a seguinte forma (lembrando que está avaliada em $\mu=0$ ):

$$
M=\left(\begin{array}{cc}
1 & \lambda \\
-2 \Psi^{\prime}(0) & 1-2 \lambda \Psi^{\prime}(0)
\end{array}\right)
$$

Então é fácil verificar que a matriz

$$
P=\left(\begin{array}{cc}
1 & 0 \\
-\Psi^{\prime}(0) & \frac{\sqrt{1-\varepsilon^{2}}}{\lambda}
\end{array}\right)
$$

com $\varepsilon:=1-\lambda \Psi^{\prime}(0)$, coloca $M$ na forma canônica de Jordan: $P^{-1} M P=\left(\begin{array}{cc}\varepsilon & \sqrt{1-\varepsilon^{2}} \\ -\sqrt{1-\varepsilon^{2}} & \varepsilon\end{array}\right)$. Assim, nas novas variáveis $\left(\begin{array}{c}\widetilde{p}_{n} \\ \widetilde{d}_{n}\end{array}\right):=P^{-1}\left(\begin{array}{c}p_{n} \\ d_{n}\end{array}\right), \operatorname{com} n \in \mathbb{N}$, a aplicação $\Phi$ se escreve como em (2.1):

$$
\begin{aligned}
\left(\begin{array}{c}
\widetilde{p}_{n} \\
\widetilde{d}_{n}
\end{array}\right) & =P^{-1} M P\left(\begin{array}{c}
\widetilde{p}_{n-1} \\
\widetilde{d}_{n-1}
\end{array}\right)+P^{-1}(\Phi-M) P\left(\begin{array}{c}
\widetilde{p}_{n-1} \\
\widetilde{d}_{n-1}
\end{array}\right) \\
& =\left(\begin{array}{cc}
\varepsilon & \sqrt{1-\varepsilon^{2}} \\
-\sqrt{1-\varepsilon^{2}} & \varepsilon
\end{array}\right)\left(\begin{array}{c}
x_{1} \\
x_{2}
\end{array}\right) \\
& +\left(\begin{array}{c}
\frac{\lambda}{\sqrt{1-\epsilon^{2}}}\left\{\Psi^{\prime}(0)\left(K_{3} x_{1}+K_{4} x_{2}\right)+\alpha\left[1-2 \Psi\left(k_{1} x_{1}+K_{2} x_{2}\right)\right]+(1-\alpha)\left[1-2 \Psi\left(K_{3} x_{1}+K_{4} x_{2}\right)\right]\right\}
\end{array}\right) \\
& -\left(\frac{\lambda}{\sqrt{1-\epsilon^{2}}}\left\{\Psi^{\prime}(0)\left(K_{3} x_{1}+K_{4} x_{2}\right)+\Psi^{\prime}(0)\left(-3+2 \lambda \Psi^{\prime}(0)\right) x_{1}+\lambda^{-1}\left(1-2 \lambda \Psi^{\prime}(0)\right) K_{4} x_{2}\right\}\right)
\end{aligned}
$$


E obtemos então que:

$$
\left(\begin{array}{l}
\widetilde{p}_{n} \\
\widetilde{d}_{n}
\end{array}\right)=\left(\begin{array}{cc}
\varepsilon & \sqrt{1-\varepsilon^{2}} \\
-\sqrt{1-\varepsilon^{2}} & \varepsilon
\end{array}\right)\left(\begin{array}{l}
x_{1} \\
x_{2}
\end{array}\right)+\left(\begin{array}{l}
g_{1}\left(x_{1}, x_{2}\right) \\
g_{2}\left(x_{1}, x_{2}\right)
\end{array}\right),
$$

onde

$$
\begin{aligned}
\left(\widetilde{p}_{n-1}, \widetilde{d}_{n-1}\right)= & \left(x_{1}, x_{2}\right), \\
K_{1}= & 1-(\lambda-J) \Psi^{\prime}(0), \\
K_{2}= & \lambda^{-1} \sqrt{1-\epsilon^{2}}(\lambda-J), \\
K_{3}= & 1-\lambda \Psi^{\prime}(0), \\
K_{4}= & \sqrt{1-\epsilon^{2}}, \\
g_{1}\left(x_{1}, x_{2}\right)= & 0, \\
g_{2}\left(x_{1}, x_{2}\right)= & \frac{\lambda}{\sqrt{1-\epsilon^{2}}}\left\{\alpha\left[1-2 \Psi\left(K_{1} x_{1}+K_{2} x_{2}\right)\right]+(1-\alpha)\left[1-2 \Psi\left(K_{3} x_{1}+K_{4} x_{2}\right)\right]+\right. \\
& \left.\Psi^{\prime}(0)\left(3-2 \lambda \Psi^{\prime}(0)\right) x_{1}-\lambda^{-1}\left(1-2 \lambda \Psi^{\prime}(0)\right) K_{4} x_{2}\right\} .
\end{aligned}
$$

Das expressões de $g_{1}$ e $g_{2}$, iremos obter os valores de $\xi_{i j}$ 's utilizando as fórmulas do Lema 2.1. Calculando todas as segundas derivadas de $g_{2}$ obtemos:

$$
\begin{aligned}
\frac{\partial^{2}}{\partial x_{1}^{2}} g_{2}\left(x_{1}, x_{2}\right) & =-\frac{2 \lambda}{\sqrt{1-\epsilon^{2}}}\left[\alpha K_{1}^{2} \Psi^{\prime \prime}\left(K_{1} x_{1}+K_{2} x_{2}\right)+(1-\alpha) K_{3}^{2} \Psi^{\prime \prime}\left(K_{3} x_{1}+K_{4} x_{2}\right)\right] \\
\frac{\partial^{2}}{\partial x_{1} \partial x_{2}} g_{2}\left(x_{1}, x_{2}\right) & =\frac{\partial^{2}}{\partial x_{2} \partial x_{1}} g_{2}\left(x_{1}, x_{2}\right)=-\frac{2 \lambda}{\sqrt{1-\epsilon^{2}}}\left[\alpha K_{1} K_{2} \Psi^{\prime \prime}\left(K_{1} x_{1}+K_{2} x_{2}\right)+(1-\alpha) K_{3} K_{4} \Psi^{\prime \prime}\left(K_{3} x_{1}+K_{4} x_{2}\right)\right] \\
\frac{\partial^{2}}{\partial x_{2}^{2}} g_{2}\left(x_{1}, x_{2}\right) & =-\frac{2 \lambda}{\sqrt{1-\epsilon^{2}}}\left[\alpha K_{2}^{2} \Psi^{\prime \prime}\left(K_{1} x_{1}+K_{2} x_{2}\right)+(1-\alpha) K_{4}^{2} \Psi^{\prime \prime}\left(K_{3} x_{1}+K_{4} x_{2}\right)\right] .
\end{aligned}
$$

Das derivadas acima e pela hipótese (3.5-a), concluímos que:

$$
\frac{\partial^{2}}{\partial x_{1}^{2}} g_{2}(0,0)=\frac{\partial^{2}}{\partial x_{1} \partial x_{2}} g_{2}(0,0)=\frac{\partial^{2}}{\partial x_{2} \partial x_{1}} g_{2}(0,0)=\frac{\partial^{2}}{\partial x_{2}^{2}} g_{2}(0,0)=0 \text {. }
$$


Desses fatos e do fato que $g_{1} \equiv 0$, teremos que $\xi_{20}=\xi_{11}=\xi_{02}=0$. Portanto falta apenas determinar $\xi_{21}$. Para isso, vamos calcular as derivadas de ordem 3 de $g_{2}$ :

$$
\begin{aligned}
\frac{\partial^{3}}{\partial x_{1}^{3}} g_{2}(0,0) & =-\frac{2 \lambda \Psi^{\prime \prime \prime}(0)}{\sqrt{1-\epsilon^{2}}}\left[\alpha K_{1}^{3}+(1-\alpha) K_{3}^{3}\right], \\
\frac{\partial^{3}}{\partial x_{1}^{2} \partial x_{2}} g_{2}(0,0) & =-\frac{2 \lambda \Psi^{\prime \prime \prime}(0)}{\sqrt{1-\epsilon^{2}}}\left[\alpha K_{1}^{2} K_{2}+(1-\alpha) K_{3}^{2} K_{4}\right], \\
\frac{\partial^{3}}{\partial x_{1} \partial x_{2}^{2}} g_{2}(0,0) & =-\frac{2 \lambda \Psi^{\prime \prime \prime}(0)}{\sqrt{1-\epsilon^{2}}}\left[\alpha K_{1} K_{2}^{2}+(1-\alpha) K_{3} K_{4}^{2}\right], \\
\frac{\partial^{3}}{\partial x_{2}^{3}} g_{2}(0,0) & =-\frac{2 \lambda \Psi^{\prime \prime \prime}(0)}{\sqrt{1-\epsilon^{2}}}\left[\alpha K_{2}^{3}+(1-\alpha) K_{4}^{3}\right] .
\end{aligned}
$$

Agora, da relação $2 \alpha J \Psi^{\prime}(0)=1$ (que é garantida pela hipótese 4), temos que $\alpha=\left(2 \Psi^{\prime}(0) J\right)^{-1}$. Isso nos permite substituir $\alpha$ por $\left(2 \Psi^{\prime}(0) J\right)^{-1}$ nas expressões das terceiras derivadas de $g_{2}$. Essa substituição simplifica um pouco mais as expressões das derivadas de ordem 3 de $g_{2}$, que agora dependerão apenas de $\lambda, J, \Psi^{\prime}(0)$ e $\Psi^{\prime \prime \prime}(0)$ :

$$
\begin{aligned}
\frac{\partial^{3}}{\partial x_{1}^{3}} g_{2}(0,0)= & \frac{\Psi^{\prime \prime \prime}(0)}{\sqrt{2 \lambda \Psi^{\prime}(0)-\lambda^{2} \Psi^{\prime}(0)^{2}}}\left\{-5 \lambda+12 \lambda^{2} \Psi^{\prime}(0)-3 \lambda J \Psi^{\prime}(0)-9 \lambda^{3} \Psi^{\prime}(0)^{2}\right. \\
& \left.+3 \lambda^{2} J \Psi^{\prime}(0)^{2}-\lambda J^{2} \Psi^{\prime}(0)^{2}+2 \lambda^{4} \Psi^{\prime}(0)^{3}\right\} \\
\frac{\partial^{3}}{\partial x_{1}^{2} \partial x_{2}} g_{2}(0,0)= & \Psi^{\prime \prime \prime}(0)\left\{-6 \lambda+2 J+\Psi^{\prime}(0)^{-1}+7 \lambda^{2} \Psi^{\prime}(0)-3 J \lambda \Psi^{\prime}(0)+J^{2} \Psi^{\prime}(0)-2 \lambda^{3} \Psi^{\prime}(0)^{2}\right\}, \\
\frac{\partial^{3}}{\partial x_{1} \partial x_{2}^{2}} g_{2}(0,0)= & \frac{\Psi^{\prime \prime \prime}(0)}{\sqrt{2 \lambda \Psi^{\prime}(0)-\lambda^{2} \Psi^{\prime}(0)^{2}}}\left[4 \lambda-2 J-12 \lambda^{2} \Psi^{\prime}(0)+7 \lambda J \Psi^{\prime}(0)+9 \lambda^{3} \Psi^{\prime}(0)^{2}\right. \\
\frac{\partial^{3}}{\partial x_{2}^{3}} g_{2}(0,0)= & \frac{\left.-3 \lambda^{2} J \Psi^{\prime}(0)^{2}-2 J^{2} \Psi^{\prime}(0)+\lambda J^{2} \Psi^{\prime}(0)^{2}-2 \lambda^{4} \Psi^{\prime}(0)^{3}\right]}{\sqrt{2 \lambda \Psi^{\prime}(0)-\lambda^{2} \Psi^{\prime}(0)^{2}}}\left[6 \lambda-6 J+2 \lambda^{-1} J^{2}-7 \lambda^{2} \Psi^{\prime}(0)+3 J \lambda \Psi^{\prime}(0)-J^{2} \Psi^{\prime}(0)+2 \lambda^{3} \Psi^{\prime}(0)^{2}\right] .
\end{aligned}
$$


Então, sabendo $g_{1} \equiv 0$, e substituindo as derivadas acima na expressão de $\xi_{21}$ dada pelo Lema 2.1. segue que:

$$
\begin{aligned}
\xi_{21} & =\frac{1}{16}\left\{\left(g_{1}\right)_{x_{1} x_{1} x_{1}}+\left(g_{1}\right)_{x_{1} x_{2} x_{2}}+\left(g_{2}\right)_{x_{1} x_{1} x_{2}}+\left(g_{2}\right)_{x_{2} x_{2} x_{2}}+i\left[\left(g_{2}\right)_{x_{1} x_{1} x_{1}}+\left(g_{2}\right)_{x_{1} x_{2} x_{2}}-\left(g_{1}\right)_{x_{1} x_{1} x_{2}}-\left(g_{1}\right)_{x_{2} x_{2} x_{2}}\right]\right\} \\
& =\frac{\Psi^{\prime \prime \prime}(0)}{16}\left[\frac{2}{\lambda} J^{2}-4 J+\frac{1}{\Psi^{\prime}(0)}+i \frac{1}{\sqrt{2 \lambda \Psi^{\prime}(0)-\lambda^{2} \Psi^{\prime}(0)^{2}}}\left(-2 \Psi^{\prime}(0) J^{2}+\left(4 \lambda \Psi^{\prime}(0)-2\right) J-\lambda\right)\right] .
\end{aligned}
$$

Temos tambem que $z_{1}(0)=\left(1-\lambda \Psi^{\prime}(0)\right)+i \sqrt{2 \lambda \Psi^{\prime}(0)-\lambda^{2} \Psi^{\prime}(0)^{2}}$. Então, substituindo os valores de $\xi_{i j}{ }^{\prime}$ s e $z_{1}(0)$ na expressão de $f_{1}(0)$ da Proposição 2.1 , temos finalmente:

$$
\begin{aligned}
f_{1}(0) & =\operatorname{Re}\left[\frac{(1-2 \lambda) z_{1}(0)^{2}}{1-z_{2}(0)} \xi_{11} \xi_{20}\right]+\frac{1}{2}\left|\xi_{11}^{2}\right|+\left|\xi_{02}^{2}\right|-\operatorname{Re}\left[z_{1}(0) \xi_{21}\right] \\
& =-\operatorname{Re}\left(z_{1}(0) \xi_{21}\right) \\
& =-\left[\frac{\Psi^{\prime \prime \prime}(0)}{16}\left(\frac{2}{\lambda} J^{2}-4 J+\frac{1}{\Psi^{\prime}(0)}\right)\left(1-\lambda \Psi^{\prime}(0)\right)-\frac{\Psi^{\prime \prime \prime}(0)}{16}\left(-2 \Psi^{\prime}(0) J^{2}+\left(4 \lambda \Psi^{\prime}(0)-2\right) J-\lambda\right)\right] \\
& =-\frac{\Psi^{\prime \prime \prime}(0)}{16}\left[\frac{2}{\lambda} J^{2}-2 J+\frac{1}{\Psi^{\prime}(0)}\right] .
\end{aligned}
$$

Portanto, temos que $-\frac{\Psi^{\prime \prime \prime}(0)}{16}>0$, pois $\Psi^{\prime \prime \prime}(0)<0$, pela hipótese $(3.5-b)$. Pelas hipóteses 3$)$ e 4) verifica-se facilmente a condição $0<\lambda \Psi^{\prime}(0)<2$. Com isso, temos que $\frac{2}{\lambda} J^{2}-2 J+\frac{1}{\Psi^{\prime}(0)}>0$, para todo valor de J. Portanto $f_{1}(0)>0$, o que prova o surgimento de uma bifurcação de Hopf supercrítica para o sistema dinâmico (3.1). Isto encerra a demonstração deste teorema.

\subsection{Simulação Numérica}

Nesta seção faremos algumas simulações numéricas para mostrar o aparecimento de oscilações periódicas no sistema dinâmico 3.1. Para isso, precisamos dos parâmetros reais $\alpha, J, \lambda$ e de uma fórmula explícita para a distribuição $\Psi(x)$. Tomemos então $\Psi(x)=\frac{\operatorname{tgh} x+1}{2}$ como função de distribuição. É simples verificar que essa função satisfaz as condições impostas para $\Psi$, no início da seção 3.1. Pelo teorema anterior, temos que uma das condições para o surgimento da bifurcação é que $0<\lambda \Psi^{\prime}(0)<2$. Como temos que $\Psi^{\prime}(0)=\frac{1}{2}$, segue que $0<\lambda<4$. Lembramos também da seção anterior que a bifurcação acontece quando $2 \alpha J \Psi^{\prime}(0)$ passa pelo valor 1 , ou equivalentemente, 
quando $\alpha J$ passa pelo valor 1. Assim, para fazermos os gráficos vamos proceder da seguinte maneira:

1) Fixar um valor de $\lambda$ entre 0 e 4 ;

2) Tomar valores para $\alpha \in(0,1)$ e $J>0$ real, de tal forma que $0<\alpha J<1$;

3) Tomar valores para $\alpha \in(0,1)$ e $J>0$ real, de tal forma que $\alpha J=1$;

4) Tomar valores para $\alpha \in(0,1)$ e $J>0$ real, de tal forma que $\alpha J>1$.

No caso 2) a origem é assintoticamente estável, e no 4) a origem perde a estabilidade, surgindo uma curva fechada, o que caracteriza uma bifurcação supercrítica. Em todos os gráficos tomamos $\left(p_{0}, d_{0}\right)=(1,1)$ e 500 iterações. Para os 4 primeiros gráficos fixamos $\lambda=0,2$ :

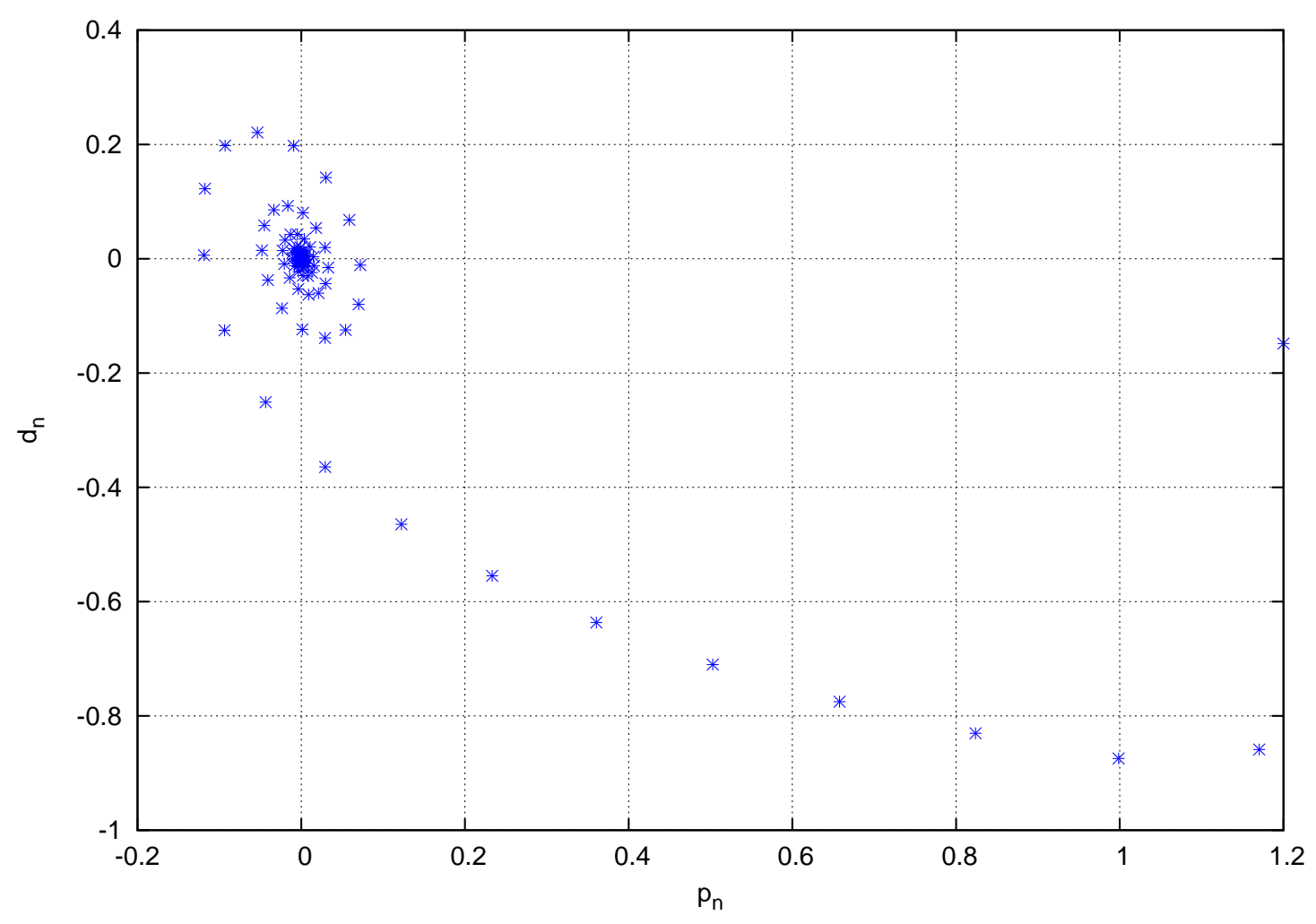

Figura 3.2: $J=1,8, \alpha=0,5$ e $\alpha J=0,9$. 


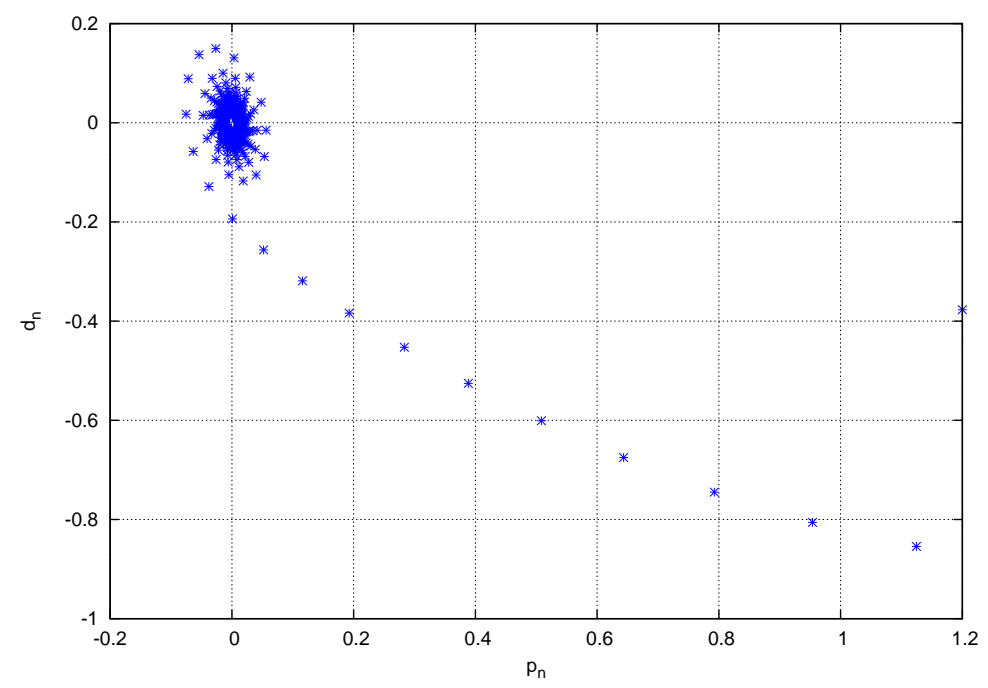

Figura 3.3: $J=4, \alpha=0,25$ e $\alpha J=1$.

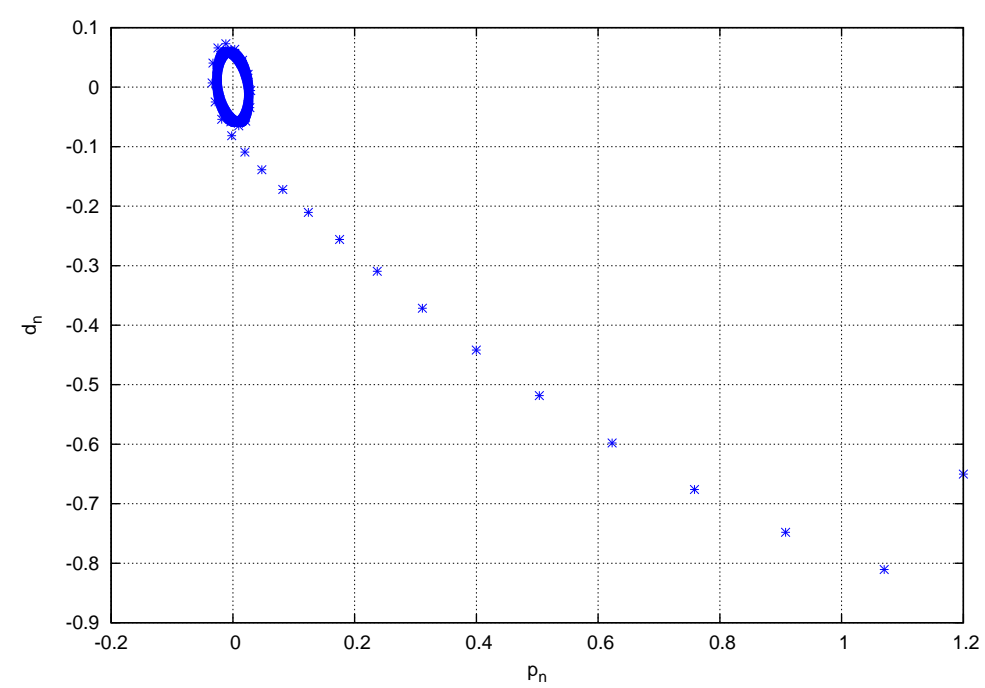

Figura 3.4: $J=11, \alpha=0,1$ e $\alpha J=1,1$. 


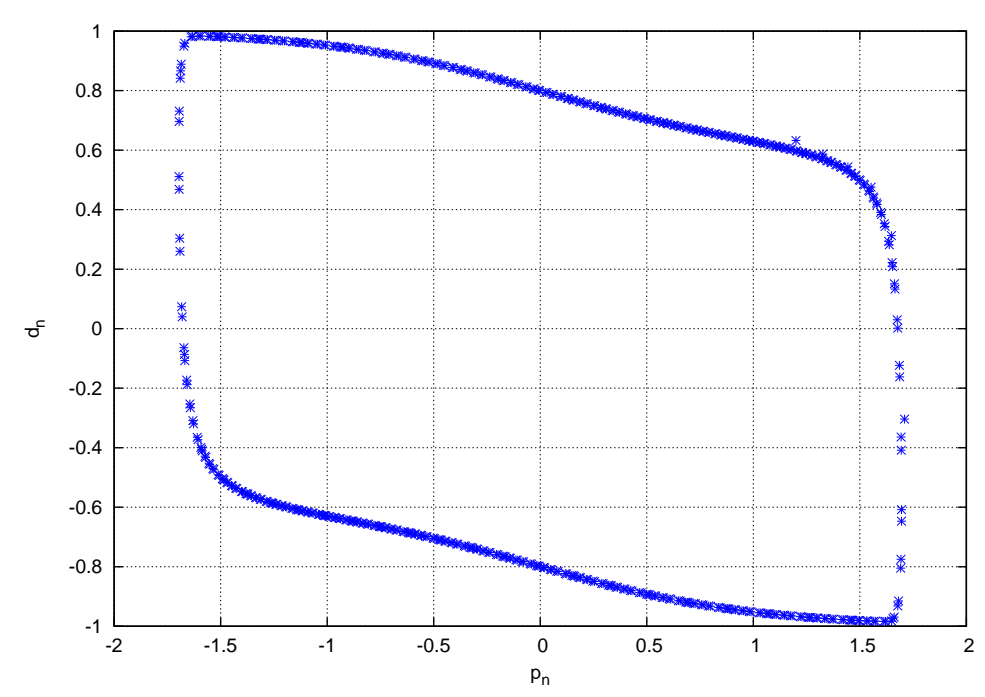

Figura 3.5: $J=5, \alpha=0,8$ e $\alpha J=4$.

Notemos que para os gráficos das figuras $3.2 \mathrm{e} 3.3$, as iterações do ponto $(1,1)$ vão para a origem, indicando assim que a origem é assintoticamente estável para $0<\alpha J \leq 1$. Já na figuras 3.4 e 3.5 vemos que a origem perde a estabilidade e as iterações do ponto $(1,1)$ convergem para uma curva fechada, quando $\alpha J>1$. Para os próximos 4 gráficos fixamos $\lambda=2$. Vamos ver como ficam os gráficos. 


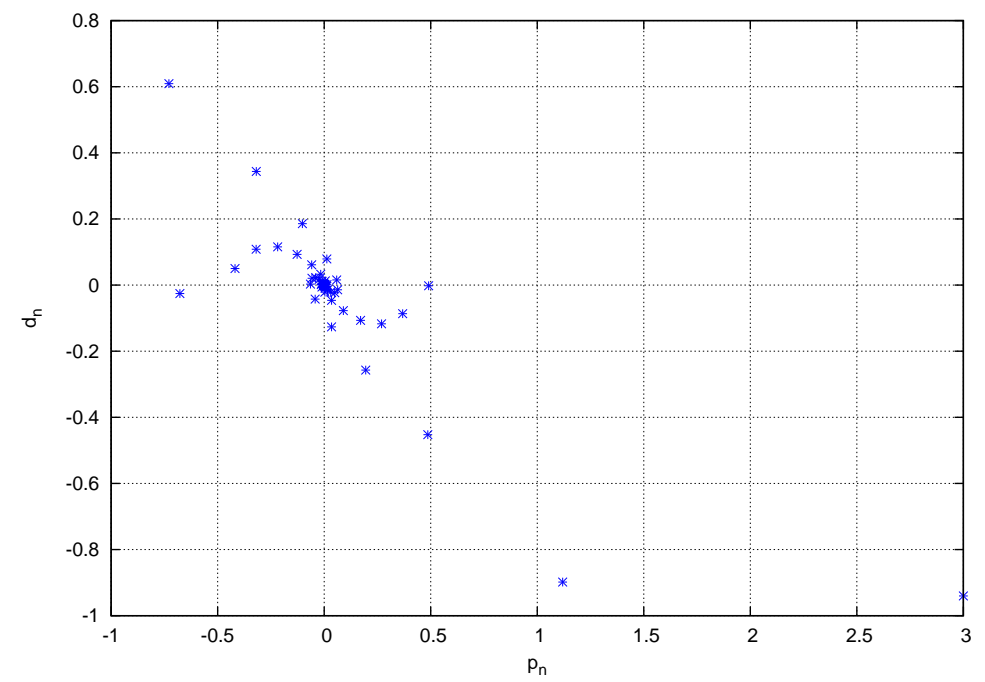

Figura 3.6: $J=1,6, \alpha=0,5$ e $\alpha J=0,8$.

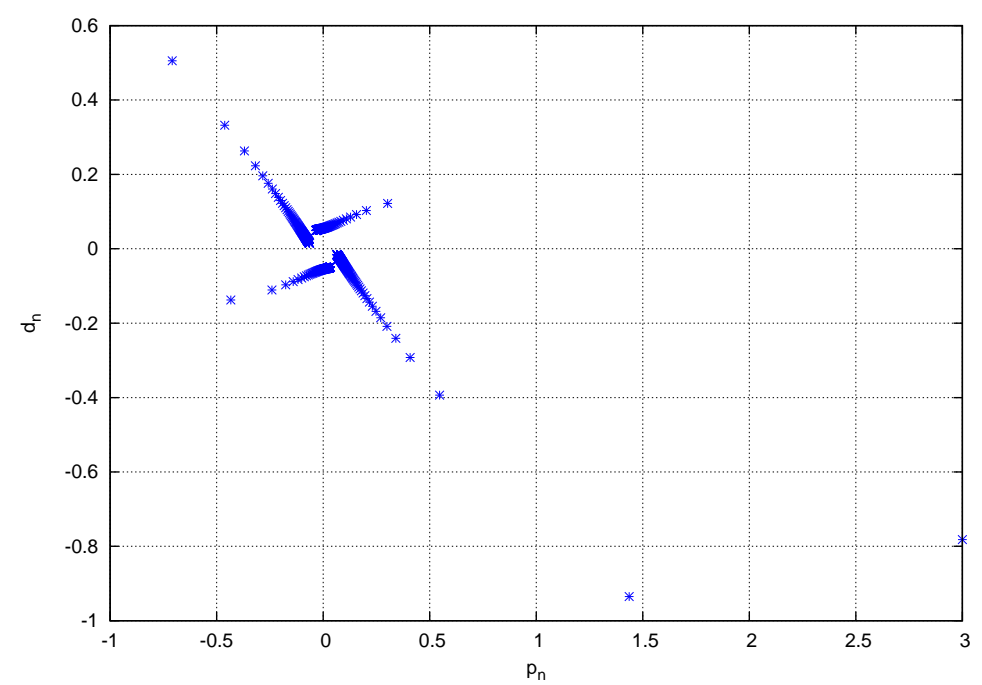

Figura 3.7: $J=2,5, \alpha=0,4$ e $\alpha J=1$. 


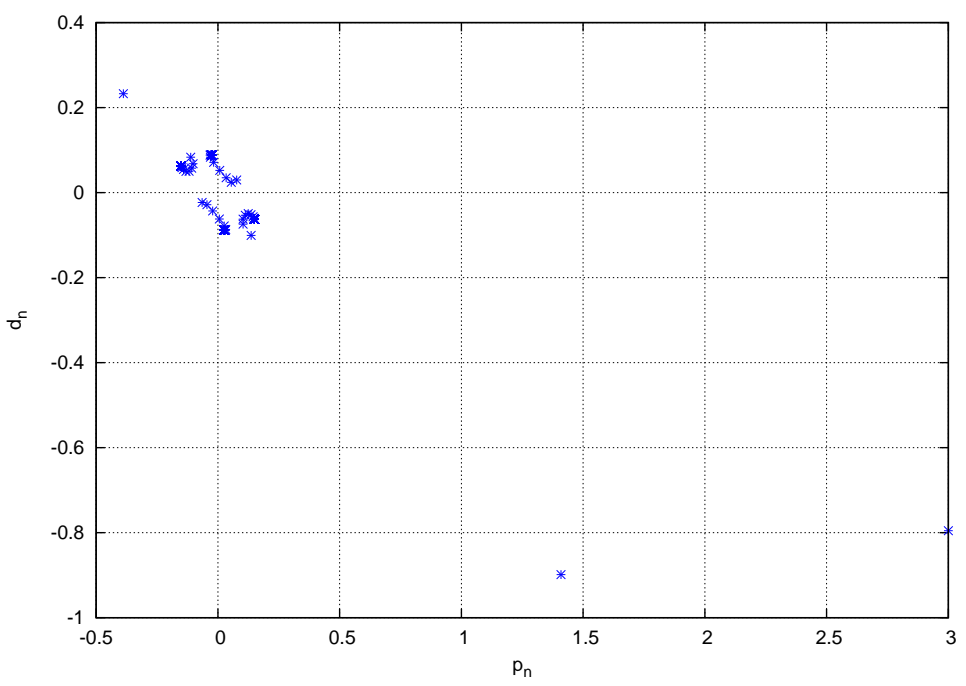

Figura 3.8: $J=12, \alpha=0,1$ e $\alpha J=1,2$.

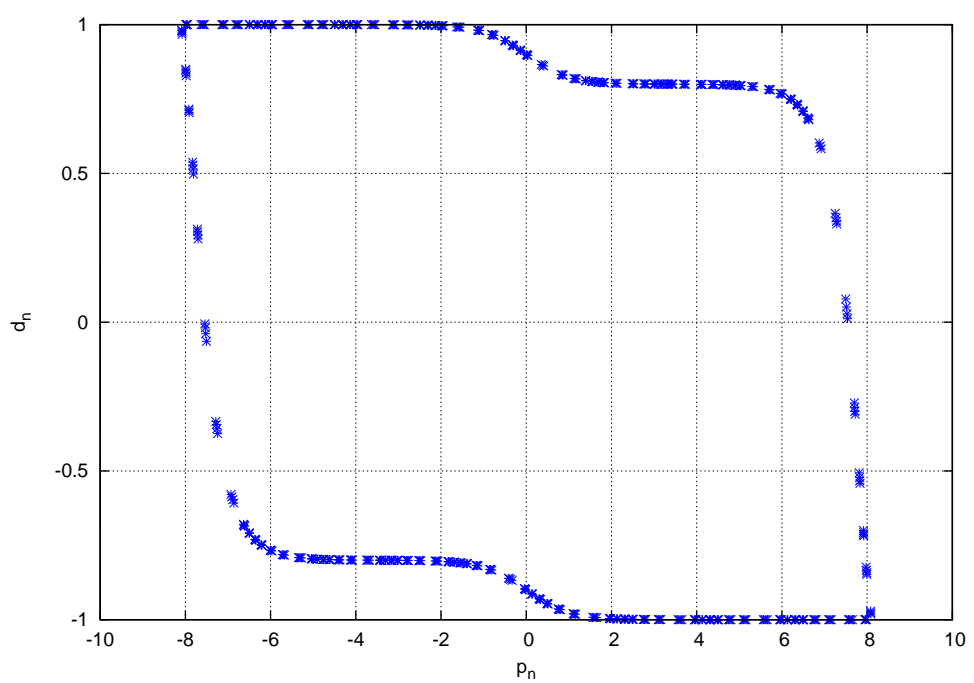

Figura 3.9: $J=10, \alpha=0,9$ e $\alpha J=9$. 
Notemos que para os gráficos das figuras 3.6 e 3.7, as iterações do ponto $(1,1)$ vão para a origem, indicando assim que a origem é assintoticamente estável para $0<\alpha J \leq 1$. Já na figuras 3.8 e 3.9 vemos que a origem perde a estabilidade e as iterações do ponto $(1,1)$ convergem para uma curva fechada quando $\alpha J>1$. Para os últimos 4 gráficos fixamos $\lambda=3,8$. Vamos ver como ficam os gráficos.

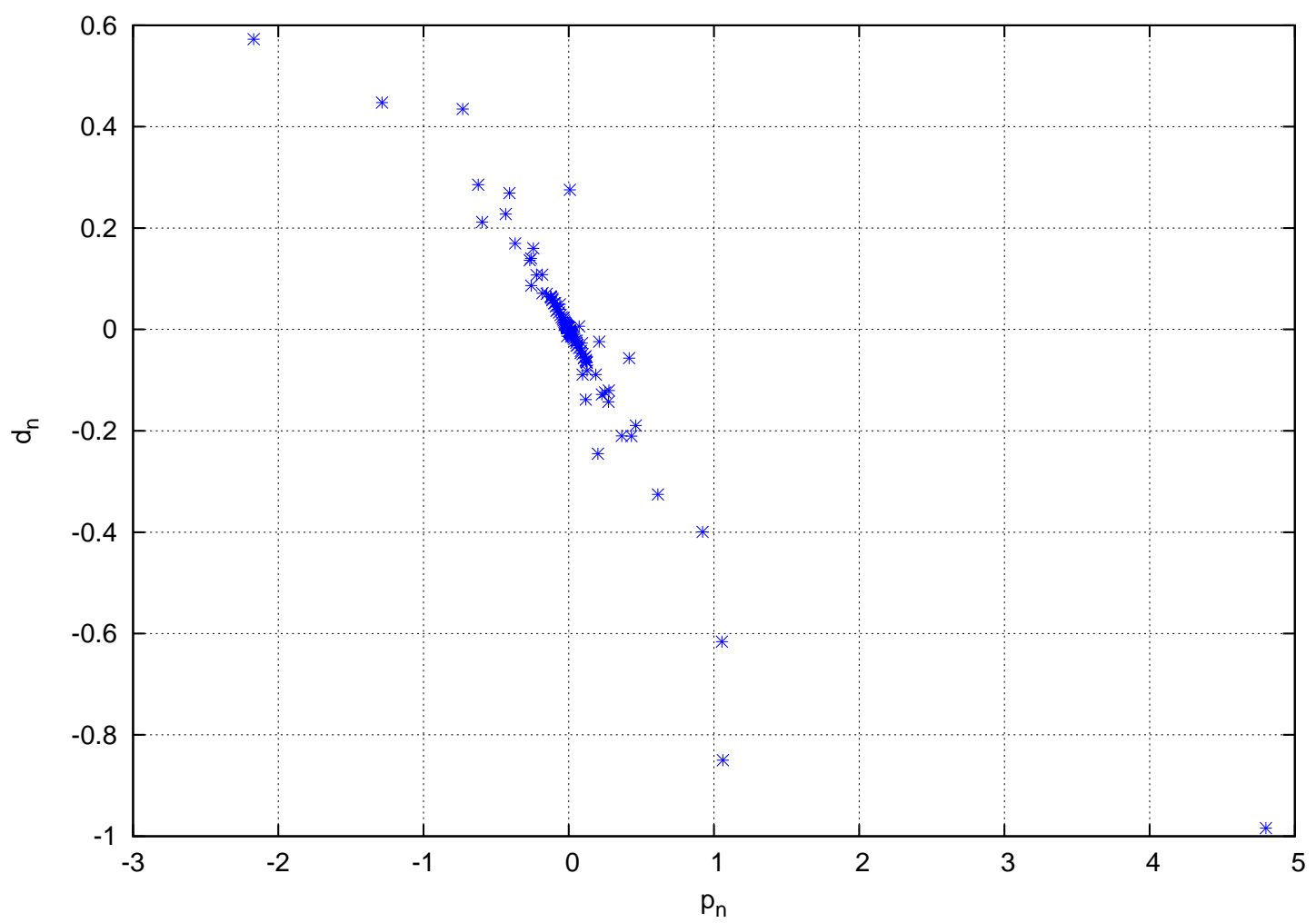

Figura 3.10: $J=3, \alpha=0,3$ e $\alpha J=0,9$. 


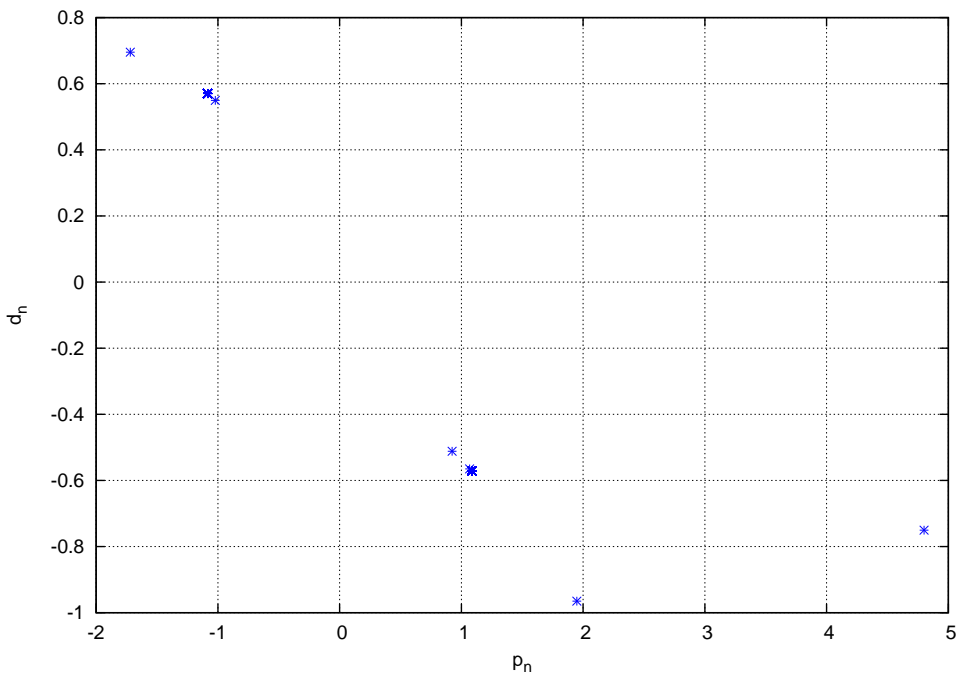

Figura 3.11: $J=8, \alpha=0,125$ e $\alpha J=1$.

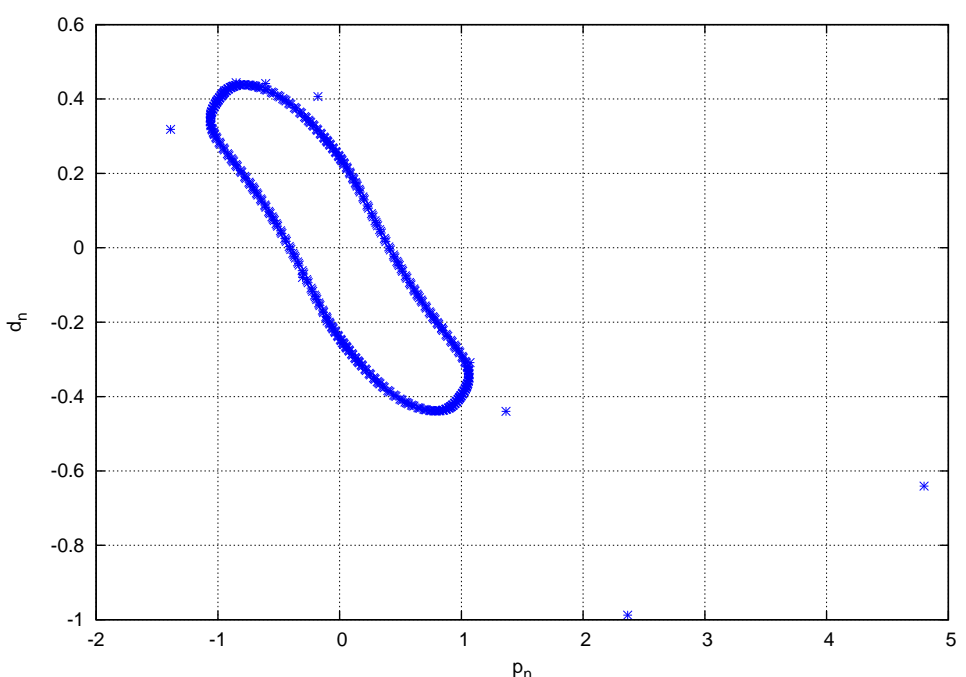

Figura 3.12: $J=5, \alpha=0,3$ e $\alpha J=1,5$. 


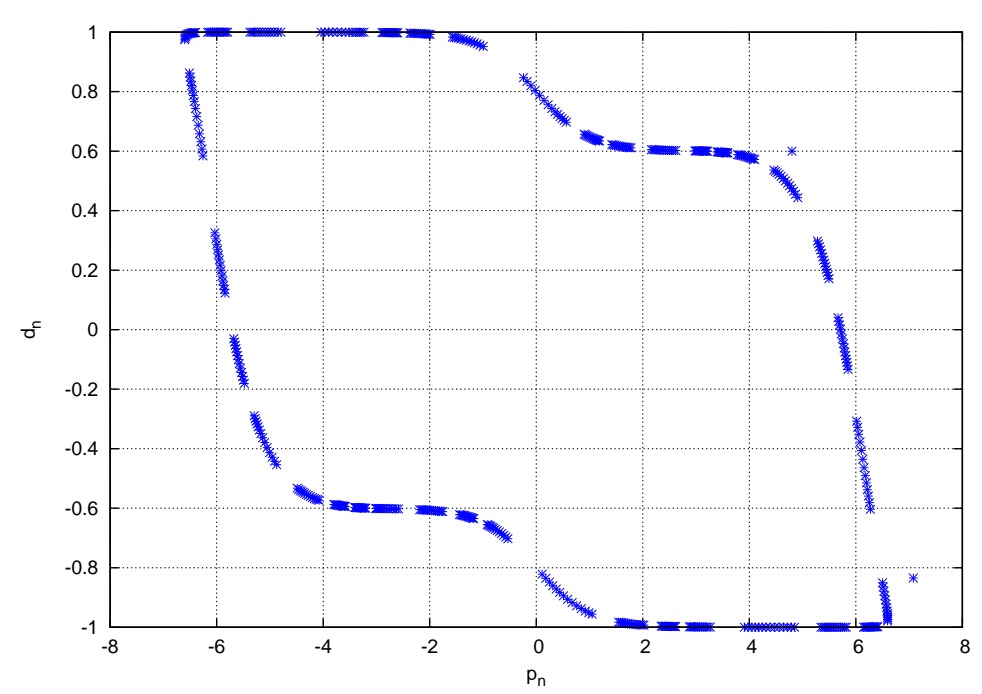

Figura 3.13: $J=10, \alpha=0,8$ e $\alpha J=8$.

Notemos que para os gráficos das figuras 3.10 e 3.11 , as iterações do ponto $(1,1)$ vão para a origem, indicando assim que a origem é assintoticamente estável para $0<\alpha J \leq 1$. Já na figuras 3.12 e 3.13 vemos que a origem perde a estabilidade e as iterações do ponto 1,1 convergem para uma curva fechada quando $\alpha J>1$.

O sistema dinâmico (3.1) ainda pode ser muito mais explorado, por exemplo estudar o comportamento das órbitas nas regiões de 2 a 6, como indicado na figura (3.1), ou ainda verificar se as curvas fechadas invariantes da região $1 \mathrm{~b}$ ) ainda persistem quando o valor de $\alpha J$ é muito grande. Como esse não é o foco dessa dissertação, deixamos essas questões para serem exploradas futuramente. 


\section{REFERÊNCIAS BIBLIOGRÁFICAS}

[1] Lanford, O. E., Bifurcation of periodic solutions into invariant tori: the work of Ruelle and Takens, Nonlinear Problems in the Physical Sciences and Biology, Lecture Notes in Mathematics, Springer-Verlag, New York, (1973), 159-192.

[2] Wan, Y. H., Computation of the stability condition for the Hopf bifurcation of difeomorphisms on $\mathbb{R}^{2}$, SIAM J. APPL. Math., vol. 34, n. 1, January 1978, 167-175.

[3] Belitsky, V.; Pereira, A. L. and Prado, F.P.A., Stability analysis with applications of a twodimensional dynamical system arising from a stochastic model for an asset market, RT-MAE 2009-03, IME-USP.

[4] Ruelle, D. and Takens, F., On the Nature of Turbulence, Commun. Math. Phys. 20 (1971), 167-192. Note Concerning our Paper, On the Nature of Turbulence, Commun. Math. Phys. 23 (1971), 343-344.

[5] Marsden, J.E. and McCracken, M., The Hopf Bifurcation and its Applications, Springer-Verlag, New York, Heidelberg, Berlin.

[6] Brin, M. and Stuck, G., Introduction to Dynamical Systems , Cambridge.

[7] Sotomayor, J. M., Lições de Equações Diferenciais Ordinárias, Projeto Euclides.

[8] Devaney, R. L., An Introduction to Chaotic Dynamical Systems, Second Edition. 